\title{
The Energetic Particle Detector
}

\section{Energetic particle instrument suite for the Solar Orbiter mission}

J. Rodríguez-Pacheco ${ }^{1}$, R. F. Wimmer-Schweingruber ${ }^{2}$, G. M. Mason ${ }^{3}$, G. C. Ho ${ }^{3}$, S. Sánchez-Prieto ${ }^{1}$, M. Prieto ${ }^{1}$, C. Martín ${ }^{2}$, H. Seifert ${ }^{3}$, G. B. Andrews ${ }^{3}$, S. R. Kulkarni ${ }^{2}$, L. Panitzsch ${ }^{2}$, S. Boden ${ }^{2}$, S. I. Böttcher ${ }^{2}$, I. Cernuda ${ }^{1}$, R. Elftmann ${ }^{2}$, F. Espinosa Lara ${ }^{1}$, R. Gómez-Herrero ${ }^{1}$, C. Terasa ${ }^{2}$, J. Almena ${ }^{1}$, S. Begley ${ }^{3}$, E. Böhm² ${ }^{2}$ J. J. Blanco ${ }^{1}$, W. Boogaerts ${ }^{2}$, A. Carrasco ${ }^{1}$, R. Castillo ${ }^{1}$, A. da Silva Fariña ${ }^{1}$, V. de Manuel González ${ }^{2}$, C. Drews ${ }^{2}$, A. R. Dupont ${ }^{3}$, S. Eldrum ${ }^{2}$, C. Gordillo ${ }^{1}$, O. Gutiérrez ${ }^{1}$, D. K. Haggerty ${ }^{3}$, J. R. Hayes ${ }^{3}$, B. Heber ${ }^{2}$, M. E. Hill ${ }^{3}$, M. Jüngling ${ }^{2}$, S. Kerem ${ }^{3}$, V. Knierim², J. Köhler ${ }^{2}$, S. Kolbe ${ }^{2}$, A. Kulemzin'², D. Lario ${ }^{26}$, W. J. Lees ${ }^{3}$, S. Liang 3 , A. Martínez Hellín ${ }^{1}$, D. Meziat ${ }^{1}$, A. Montalvo ${ }^{1}$, K. S. Nelson ${ }^{3}$, P. Parra ${ }^{1}$, R. Paspirgilis ${ }^{2}$, A. Ravanbakhsh ${ }^{2}$, M. Richards ${ }^{2}$, O. Rodríguez-Polo ${ }^{1}$, A. Russu ${ }^{1}$, I. Sánchez ${ }^{1}$, C. E. Schlemm ${ }^{3}$, B. Schuster ${ }^{2}$, L. Seimetz ${ }^{2}$,

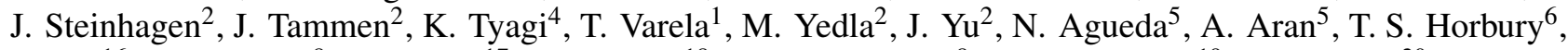
B. Klecker ${ }^{16}$, K.-L. Klein ${ }^{8}$, E. Kontar ${ }^{17}$, S. Krucker ${ }^{18}$, M. Maksimovic ${ }^{8}$, O. Malandraki ${ }^{19}$, C. J. Owen ${ }^{20}$, D. Pacheco ${ }^{5}$, B. Sanahuja ${ }^{5}$, R. Vainio ${ }^{21}$, J. J. Connell ${ }^{11}$, S. Dalla ${ }^{22}$, W. Dröge ${ }^{23}$, O. Gevin ${ }^{24}$, N. Gopalswamy ${ }^{26}$, Y. Y. Kartavykh ${ }^{23,28}$, K. Kudela ${ }^{25}$, O. Limousin ${ }^{24}$, P. Makela ${ }^{26}$, G. Mann ${ }^{27}$, H. Önel ${ }^{27}$, A. Posner ${ }^{12}$, J. M. Ryan ${ }^{11}$, J. Soucek ${ }^{10}$, S. Hofmeister ${ }^{9}$, N. Vilmer ${ }^{8}$, A. P. Walsh ${ }^{7}$, L. Wang ${ }^{13}$, M. E. Wiedenbeck ${ }^{15}$, K. Wirth ${ }^{14}$, and Q. Zong ${ }^{13}$

(Affiliations can be found after the references)

Received 15 February 2019 / Accepted 15 April 2019

\begin{abstract}
After decades of observations of solar energetic particles from space-based observatories, relevant questions on particle injection, transport, and acceleration remain open. To address these scientific topics, accurate measurements of the particle properties in the inner heliosphere are needed. In this paper we describe the Energetic Particle Detector (EPD), an instrument suite that is part of the scientific payload aboard the Solar Orbiter mission. Solar Orbiter will approach the Sun as close as 0.28 au and will provide extra-ecliptic measurements beyond $\sim 30^{\circ}$ heliographic latitude during the later stages of the mission. The EPD will measure electrons, protons, and heavy ions with high temporal resolution over a wide energy range, from suprathermal energies up to several hundreds of megaelectronvolts/nucleons. For this purpose, EPD is composed of four units: the SupraThermal Electrons and Protons (STEP), the Electron Proton Telescope (EPT), the Suprathermal Ion Spectrograph (SIS), and the HighEnergy Telescope (HET) plus the Instrument Control Unit that serves as power and data interface with the spacecraft. The low-energy population of electrons and ions will be covered by STEP and EPT, while the high-energy range will be measured by HET. Elemental and isotopic ion composition measurements will be performed by SIS and HET, allowing full particle identification from a few kiloelectronvolts up to several hundreds of megaelectronvolts/nucleons. Angular information will be provided by the separate look directions from different sensor heads, on the ecliptic plane along the Parker spiral magnetic field both forward and backwards, and out of the ecliptic plane observing both northern and southern hemispheres. The unparalleled observations of EPD will provide key insights into long-open and crucial questions about the processes that govern energetic particles in the inner heliosphere.
\end{abstract}

Key words. acceleration of particles - instrumentation: detectors - Sun: coronal mass ejections (CMEs) - Sun: flares - Sun: heliosphere Sun: particle emission

\section{Introduction}

The Solar Orbiter Mission (Müller \& Marsden 2013; Müller et al. 2020) is a collaboration between ESA and NASA. The mission aims to understand how the Sunv creates and controls the heliosphere, which entails answering the following questions:

1. What drives the solar wind and where does the coronal magnetic field originate from?

2. How do solar transients drive heliospheric variability?

3 . How do solar eruptions produce energetic particle radiation that fills the heliosphere?

4. How does the solar dynamo work and drive connections between the Sun and the heliosphere?
Solar Orbiter's heliocentric orbit has a perihelion of $0.28 \mathrm{au}$. It provides a unique opportunity to make in-situ measurements of the plasma properties, electromagnetic fields, and energetic particles in the inner heliosphere, which are less distorted by transport effects. In contrast to previous and contemporary missions approaching the Sun, such as Helios (Porsche 1977) and Parker Solar Probe (PSP, Fox et al. 2016), Solar Orbiter has a comprehensive set of remote-sensing and in-situ instruments that will allow, for the first time, a direct correlation between in-situ measurements and high-resolution imaging and spectroscopic observations of the solar source regions from a close vantage point. During the final part of the mission, Solar Orbiter will also provide novel remote-sensing observations of the solar polar regions. For further details on the spacecraft and the mission, we 
refer the reader to Müller \& Marsden (2013), Müller et al. (2020) and García-Marirrodriga et al. (2020).

The Solar Orbiter scientific payload has been designed to provide the combined measurements required to achieve the science goals of this mission. The in-situ payload is comprised of the Magnetometer (MAG, Horbury et al. 2020), the Radio and Plasma Wave analyser (RPW, Maksimovic et al. 2020), the Solar Wind Analyser (SWA, Owen et al. 2020) and the Energetic Particle Detector (EPD). The remote-sensing instrumentation is composed by the X-ray Spectrometer (STIX, Krucker et al. 2020), the Polarimetric and Helioseismic Imager (PHI, Solanki et al. 2020), the Extreme Ultraviolet Imager (EUI, Rochus et al. 2020), the Spectral Imaging of the Coronal Environment instrument (SPICE, SPICE Consortium 2020), the visible light and ultraviolet coronal imager (METIS, Antonucci et al. 2020), and the Heliospheric Imager (SoloHI, Howard et al. 2020).

The mission scientific objectives require a high level of coordination among the instruments in order to maximise the scientific return. For this purpose, a set of coordinated activities has been devised and compiled into the so-called Science Activity Plan (SAP, Zouganelis et al. 2020). In-situ measurements are coordinated by the in-situ working group (Walsh et al. 2020). Similarly, a remote-sensing working group (Auchère et al. 2020) has been established to coordinate remote-sensing observations. Coordination with other assets such as the Parker Solar Probe (Fox et al. 2016) has been addressed by Velli et al. (2020). Finally a Modelling And Data Analysis Working Group (MADAWG) has been established (Rouillard et al. 2020) to assist the mission planning and scientific exploitation with dedicated tools.

The EPD measurements will be central in addressing the mission top-level question pertaining to energetic particles. It will also provide supporting measurements to address the other mission scientific questions. Furthermore, unveiling the origins of solar energetic particles (SEP) will require complementary high-cadence remote-sensing observations of the solar sources at multiple wavelengths (from X-ray to visible light), their radio signatures, and a continuous monitoring of the plasma and magnetic field environment around the spacecraft.

Energetic particles are a fundamental ingredient of the dynamical Sun. A major part of the energy released during eruptive events in the solar atmosphere is transferred to particles (Emslie et al. 2012), and then carried into the heliosphere. Nonthermal particle populations are also a possible basic ingredient of the hot corona; understanding them gives insight into the elusive heating process.

The heliosphere constitutes a unique laboratory to probe energetic particles in an astrophysical setting because in-situ measurements of the particles can be conducted. An additional information can be obtained from the coronal plasma environment (from where the particles originate and propagate) and its dynamics, which can be imaged and probed by spectroscopy. Finally, shock waves, which are one of the prime particle accelerators, can also be measured in-situ, together with the associated wave and particle populations. All this can be done with a time resolution that reveals properties of the processes of acceleration and transport.

This work describes the EPD instrument suite, which is made up of multiple sensors: the SupraThermal Electron and Proton (STEP) sensor, the Electron-Proton Telescope (EPT), the Suprathermal Ion Spectrograph (SIS), and the High-Energy Telescope (HET). These instruments have been designed to measure the physical properties of the different energetic particle populations in the inner heliosphere. The structure of the paper is as follows: Sect. 2 details the EPD scientific goals; a general description of the EPD suite, its components, and its capabilities are provided in Sect. 3; the scientific sensors of EPD are described in detail in Sects. 4-7; finally the Instrument Control Unit (ICU) and the onboard processing software are presented in Sect. 8.

\section{Science objectives of the Energetic Particle Detector}

The Sun is the most powerful source of energetic particles in the Solar System. Transient processes on the solar surface and in the solar atmosphere are capable of energising electrons (up to tens of $\mathrm{MeV}$ ) and ions (up to a few $\mathrm{GeV}$ ) that can escape to the interplanetary space along open magnetic field lines (see e.g. Klein \& Dalla 2017). These particles, ranging from suprathermal ( $\sim$ few $\mathrm{keV}$ ) to relativistic energies, can be observed in-situ as SEP events by radiation detectors in space. Solar flares and coronal mass ejections (CMEs) are the main sources of SEPs. Solar energetic particle events are also of great relevance for space weather because they can cause large radiation increases in interplanetary space and over the polar regions of the Earth, potentially harmful for human crews in space and for space-based technology (e.g. Chenette et al. 1994; Mewaldt 2006; Vainio et al. 2009; Jiggens et al. 2014; McKenna-Lawlor et al. 2015).

Solar energetic particle events are often classified into two categories, impulsive and gradual (Cane et al. 1986; Reames 1999), which show different observational characteristics related to different acceleration sites. At moderate energies, gradual events show abundance ratios and charge states similar to the ambient corona and the interplanetary medium (Klecker et al. 2007; Desai \& Giacalone 2016, and references therein). They are proton rich and show larger fluences and longer durations (up to several days); they also tend to be associated with fast and large CMEs driving shock waves in the corona and the interplanetary medium, often accompanied by observational signatures such as type II radio bursts. Impulsive events have typically much shorter durations (hours) and lower fluences. They are electron-rich and show higher charge states (Klecker et al. 1984) and dramatic enhancements of the ${ }^{3} \mathrm{He} /{ }^{4} \mathrm{He}$ ratio with respect to the coronal values, as well as enrichments in Fe and other heavy elements (Reames et al. 1994; Mason 2007; Reames 2018). Impulsive events appear in association with type III radio bursts and often with solar flares, narrow CMEs, and extreme-ultraviolet (EUV) jets (Kahler et al. 2001; Nitta et al. 2006; Bučík et al. 2018). Recent observations suggest that EUV coronal waves may also affect the injection of ${ }^{3} \mathrm{He}$-rich SEPs into interplanetary space during some ${ }^{3} \mathrm{He}$-rich events (Bučík et al. 2015, 2016).

Regarding the acceleration mechanisms, impulsive events involve acceleration of hot flaring plasma, via electric fields or stochastic acceleration, over short timescales at spatially compact sources (the reconnection site at solar flares). They may also include resonant acceleration processes responsible for the selective enhancements of certain species (Reames 2013, and references therein). Gradual events involve long-lasting acceleration of the ambient plasma at spatially extended CME-driven shocks propagating through the corona and the interplanetary medium (Lee et al. 2012, and references therein). Nevertheless, making a clear distinction between acceleration mechanisms in impulsive and gradual SEP events may be difficult because different processes may play a role in accelerating particles during the development of an event (e.g. Klein \& Dalla 2017, and 
references therein). Some SEP events may exhibit mixed or hybrid (Kallenrode et al. 1992) composition, that is, with contribution of particles accelerated both at flares and at CME-driven shocks. The different scenarios proposed to explain the presence of flareaccelerated particles in large SEP events are detailed in the recent papers by Desai \& Giacalone (2016) and Kartavykh et al. (2016).

While it appears plausible that some aspects of the spatial SEP distribution observed in the interplanetary medium are greatly influenced by the size of the acceleration site (e.g. Lario et al. 2014; Gómez-Herrero et al. 2015), the existence of SEP events that fill all solar longitudes, and the faster spread of electrons over protons, which is inconsistent with the expansion of a single acceleration region (Kallenrode 1993a; Richardson et al. 2014), pose challenges to this view. In particular, a single expansion of an acceleration region cannot explain the inferred differences in azimuthal spread between electrons and protons (see, e.g. Figs. 15 and 16 in Richardson et al. 2014). During impulsive SEP events, energetic particles were thought to fill only relatively narrow angular ranges, corresponding to the region well-connected to a spatially compact source. However, recent STEREO observations (Wiedenbeck et al. 2013) show that sometimes ${ }^{3} \mathrm{He}$-rich events can also show relatively wide particle spreads. For further details on the candidate physical processes that may be responsible for the spatial spread of SEPs in the interplanetary medium, see for example Dresing et al. (2012, 2014), Gómez-Herrero et al. (2015), Lario et al. (2016), and Strauss et al. (2017) and references therein.

In-situ observations of SEPs provide the opportunity to study all aspects in the development of SEP events, namely the acceleration processes, particle escape from the source, and propagation through the interplanetary medium. Moreover, energetic electrons and ions can also serve as tracers for the magnetic connectivity of the location where the measurement takes place.

The EPD aims to provide answers to the third overarching science question of Solar Orbiter: How do solar eruptions produce energetic particle radiation that fills the heliosphere? This can be broken into three top-level sub-questions:

1. Injection: How and where are energetic particles injected at the sources and, in particular, what are the seed populations for energetic particles?

2. Acceleration: How and where are energetic particles accelerated at the Sun and in the interplanetary medium?

3. Transport: How are energetic particles released from their sources and distributed in space and time?

\subsection{Injection}

The material actually energised by CME-driven shocks is called the seed population. The charge states observed during gradual events seem to be consistent with the temperature of the ambient corona and the solar wind, rather than hot plasma from flaring active regions, at least for energies below several tens of megaelectronvolts. However, the composition often shows peculiarities such as moderate enhancements of rare species such as ${ }^{3} \mathrm{He}$ and $\mathrm{He}^{+}$, suggesting a seed population drawn from the suprathermal ion pool (ions $\gtrsim 2 \mathrm{keV}$ ), which is always present (Fisk \& Gloeckler 2008) but is characterised by stronger variability in both intensity and composition than the ambient solar wind. The origin of these suprathermal ions is not fully understood and could include remnants from previous SEP events and stream interaction regions (SIRs) or an almost continuous solar or interplanetary source. Furthermore, pickup $\mathrm{He}^{+}$ions of interstellar origin, with their velocity distribution function (VDF) being very different from the solar wind, provide an additional suprathermal source, making them ideal to probe injection and acceleration processes that depend on mass per charge and on the VDF (e.g. Kucharek et al. 2003, and references therein).

The combination of SWA and EPD measurements (particularly the STEP and SIS sensors) will provide the composition and energy spectrum (or VDF) ranging from solar wind to suprathermal energies at different heliocentric distances. These data constitute an essential input for models of SEP acceleration at coronal and interplanetary shock waves (e.g. Tylka \& Lee 2006; Neergaard Parker \& Zank 2012; Vainio et al. 2014; Afanasiev et al. 2015), and are also fundamental to understanding the origin of the suprathermal pool itself. Measurements close to the Sun will be particularly important because the SEP composition will be much less disturbed by interplanetary transport processes, enabling a more direct study of questions such as the influence of the shock obliquity on the injection and acceleration processes (Tylka et al. 2005; Battarbee et al. 2013) or the amount of direct flare contributions during large gradual events (Cane et al. 2010; Tylka et al. 2013).

\subsection{Acceleration mechanisms}

Impulsive SEP events are associated with solar type III radio bursts. Many clearly come from flares/microflares accompanied by narrow CMEs or EUV jets (Kahler et al. 2001; Nitta et al. 2006; Bučík et al. 2018), but often not even a microflare or soft X-ray burst is observed. The acceleration is thought to be a consequence of magnetic reconnection processes in the low corona. The physical mechanisms may involve direct electric fields, stochastic acceleration, and resonant wave-particle interactions. Resonant acceleration processes could explain the preferential acceleration of some species, which produce the composition anomalies observed in-situ. Solar Orbiter will provide the opportunity to study impulsive SEP events close to the Sun. The SIS and HET will obtain accurate measurements of the isotopic and elemental composition, while STEP, EPT, and HET will detect the associated energetic electrons released from the reconnection site, enabling an accurate determination of the solar release time. These EPD in-situ observations will be combined with radio (Pick \& Vilmer 2008; Kontar et al. 2017), X-ray, and especially EUV observations with high cadence and spatial resolution, enabling an unambiguous identification of the associated solar activity phenomena and the location of the acceleration site. In particular, the combination of EPD and STIX will allow for a comparison between the electron spectrum at the acceleration region inferred from X-ray measurements and the electron spectrum observed in-situ (Krucker et al. 2007).

Particle acceleration at CME-driven shocks is widely seen as the main process in gradual SEP events (e.g. Reames 1999). The main particle shock acceleration processes are shock-drift acceleration, shock surfing acceleration and diffusive shock acceleration (e.g. Vainio \& Afanasiev 2018). The latter, a first-order Fermi acceleration mechanism, is commonly used to describe SEP shock-acceleration (e.g. Lee et al. 2012). This acceleration process is influenced by the shock properties, by the seed population available for acceleration, and the ambient turbulence around the shock (e.g. Rodríguez-Pacheco et al. 1998; Lee et al. 2012). Self-generated waves produced by the accelerated SEP population also play a fundamental role in the development of the turbulence that is key to reaching the observed particle energies (Vainio \& Laitinen 2007). According to some studies, the presence of previous CMEs may also play an important role in SEP acceleration during gradual events (Kahler 2001; Gopalswamy et al. 2002). 
In gradual events, the shock-acceleration processes start when the shock is formed in the corona and continue as it propagates through the outer coronal layers and the interplanetary medium. In general, the stronger acceleration occurs when the shock is still close to the Sun. The shape of the SEP time profile is greatly influenced by the location of the observer with respect to the central part of the shock (e.g. Cane et al. 1988; Heras et al. 1995; Lario et al. 1998; Aran 2007). When the interplanetary shock approaches the location of the observer, particle fluxes of low to moderate energies often show an increase and peak locally at the passage time of the shock, forming a socalled energetic storm particle (ESP) event (Bryant et al. 1962). The EPD will observe gradual events and will sample the associated ESP events close to the Sun before the shock has been weakened by the interplanetary propagation. The EPD observations will be combined with those from MAG, RPW, and SWA to determine local shock and turbulence properties, often with the highest time cadence available (burst mode, see Sect. 3).

The observational distinction of gradual and impulsive events becomes less and less clear when high energies (100s-1000s of MeV) are considered. Statistical evidence (e.g. Dierckxsens et al. 2015; Trottet et al. 2015) and timing studies of ground level enhancements (GLEs; e.g. Klein \& Dalla 2017, and references therein) suggest that acceleration processes related to the flare or to the restructuring of the magnetically stressed corona in the wake of a CME could be a plausible alternative to shock acceleration. The timing of the observed SEP signatures will carry detailed structure that is blurred at 1 au (see Sect. 2.3), and will allow for a much more detailed comparison with particle acceleration signatures from the corona observed in HXR and radio.

Besides the previously mentioned sources of accelerated particles, recent observations of energetic particle enhancements in turbulent wakes of interplanetary shocks, near reconnecting current sheets, and within magnetic cavities filled with magnetic islands strongly support theoretical expectations of particle energisation to kilo- or even megaelectronvolt energies, in the presence of coherent structures in the solar wind (Zank et al. 2014, 2015; le Roux et al. 2015, 2016). Many examples of such atypical energetic particle enhancements (AEPEs) in the heliosphere at radial distances $\geq 1$ au have been reported (Khabarova et al. 2018, and references therein). The EPD observations combined with MAG and SWA will shed further light on the effects of these local particle acceleration processes inside of 1 au in the inner heliosphere.

\subsection{Transport}

Solar energetic particle events are routinely observed in situ by several spacecraft located near 1 au. These measurements can only reveal limited information about the source of particles when these are located in the solar corona or travelling into the interplanetary medium but far from $1 \mathrm{au}$. The reason for the difficulty in inferring the characteristics of the distant particle sources is linked to the effects of the transport of particles from their source to the position of the observer. On one hand, complications arise because observations are made several particle scattering lengths from the source, and therefore its characteristics are blurred by transport processes. Helios observations already illustrated this by showing multiple SEP events at 0.31 au that appeared as a single event at 1 au (Wibberenz \& Cane 2006; Agueda \& Lario 2016). On the other hand, the effect of adiabatic deceleration may result in particles $\lesssim 200 \mathrm{keV} \mathrm{n}^{-1}$ being unobserved at $1 \mathrm{au}$ (Mason et al. 2012). By travelling as close to the Sun as $60 R_{\odot}$ with an advanced in-situ and remote sensing payload, Solar Orbiter will revolutionise our understanding of SEPs. The energy resolution of the STEP, EPT, and SIS detectors will permit the detailed analysis of the seed particle population in acceleration processes, since the effect of adiabatic deceleration at such short heliocentric distances is reduced (e.g. Ruffolo 1995; Kocharov et al. 1998; Wijsen et al. 2019). Also, transport effects might be diminished at close perihelion distances, where Solar Orbiter will be within a few to $\sim 1$ scattering mean free paths (see, e.g. Kallenrode 1993b; Wibberenz \& Cane 2006; Pacheco et al. 2019). This permits more accurate source identification and timing studies, and will ultimately allow us to distinguish between various particle acceleration mechanisms that are otherwise impossible to discern. The data that EPD will provide (with a temporal resolution up to $1 \mathrm{~s}$ when set in burst mode), along with the local shock and turbulence parameters that can be derived from the data provided by MAG, SWA, and RPW, will allow for theory and models of the acceleration processes to be greatly improved.

When energetic particles are released from their acceleration sites, namely flares or CME-driven shocks, they propagate predominantly along the interplanetary magnetic field (IMF), which at large spatial scales is often assumed to be ordered as a Parker spiral, with turbulent fluctuations at smaller spatial scales. Understanding how energetic particles propagate in the solar wind from their sources to the point of observation is not only essential to better understanding the acceleration processes at or near the Sun, but is also an important problem in its own right. We note, for instance, that even in the case of impulsive events observed by spacecraft separated by short longitudinal distances, transport conditions can vary significantly, yielding different in-situ particle intensity-time profiles (Pacheco et al. 2017). As detailed in the following sections, EPD instruments will provide numerous energy channels for a large variety of particle species, as well as directional information of particle intensities given by the four fields of view of the EPT and HET telescopes. Alone and in combination with measurements from other spacecraft, EPD observations will not only help better constrain current particle transport models that describe SEP events (e.g. Kozarev et al. 2013; Schwadron et al. 2014; RodríguezGasén et al. 2014; Pomoell et al. 2015; Luhmann et al. 2017; Hu et al. 2017; Afanasiev et al. 2018), but in addition, the out of ecliptic orbits of Solar Orbiter will provide a unique observational vantage point to assess the importance of propagation across the magnetic field in SEP transport. Among possible mechanisms that may play a role in perpendicular transport are turbulence-associated cross field motion (e.g. Zhang et al. 2009; Schwadron et al. 2010; Dröge et al. 2010; Kartavykh et al. 2016; Laitinen et al. 2016; Wijsen et al. 2019), charged particle drifts (Dalla et al. 2017), and the influence of the heliospheric current sheet (e.g. Agueda et al. 2013; Battarbee et al. 2018). Magnetic field line meandering due to turbulence gives SEPs fast access to a wide range of longitudes near the Sun (Laitinen et al. 2016). Charged particle drifts are charge-to-mass-ratio dependent and would result in Fe/O time dependence and other compositional characteristics being different near the Sun compared to 1 au (e.g. Dalla et al. 2017). Comparison between Solar Orbiter and 1 au data will be key to discerning how the various crossfield transport mechanisms affect different particle species and energies.

Electron beams responsible for type III bursts generate plasma waves (Ergun et al. 1998) that are affected by density inhomogeneities (Kontar 2001; Voshchepynets et al. 2015). The excellent energy resolution of STEP/EPD will allow us to 
understand how the electron spectrum evolves in the heliosphere and to compare it with various model predictions for the inner heliosphere (Dröge et al. 2010; Kontar \& Reid 2009; Reid \& Kontar 2013).

In combination with vector magnetic field and plasma measurements, energetic particles can be used as probes to study the large-scale structure of the coronal magnetic fields and the 3D structure of magnetohydrodynamic (MHD) turbulence in the near-Sun environment. Propagation of charged particles parallel to the IMF is affected by a number of processes, particularly the adiabatic motion along the smooth large-scale field and the pitch angle scattering off the small-scale irregularities (see, e.g. Aran et al. 2018). One crucial parameter is the pitch-angle diffusion coefficient $D_{\mu \mu}$ of the particle, which can be related to the its scattering mean free path if the power spectrum of the turbulence is known (e.g. Hasselmann \& Wibberenz 1970; Schlickeiser 1989). However, because most power spectra are obtained at $1 \mathrm{au}$, assumptions about the radial dependence of the turbulent magnetic field must be made (e.g. Mason et al. 2012, and references therein). The properties and evolution of turbulence depend on the plasma properties, which change across the heliosphere and introduce many of the uncertainties in our understanding of the SEP transport problem. This picture will be altered significantly when Solar Orbiter travels to $0.28 \mathrm{au}$. With the help of the vector magnetic field and plasma measurements of the Solar Orbiter, it will be possible to map the power spectrum of the turbulent magnetic field as a function of heliocentric distance, which can be used to constrain the parameter space of the current transport models and hence improve their reliability.

Finally, solar wind conditions, and hence transport effects, together with the evolution of the efficiency of CME driven shocks at accelerating particles and the magnetic connectivity between the observer and the source, play a major role in determining the variation of the peak intensity and fluence with the heliocentric radial distance during SEP events (Aran et al. 2005, 2006; Lario et al. 2006, 2007, 2013; Vainio et al. 2007; He et al. 2017). The EPD measurements of SEP events at distances of $<1$ au in combination with observations at 1 au by other spacecraft will provide essential information for SEP interplanetary environment models (e.g. Kozarev et al. 2010; Crosby et al. 2015; Marsh et al. 2015).

\subsection{Additional science targets}

In addition to the primary science targets, there are other important topics that EPD will be able to investigate during the mission, including the following.

- EPD will provide the first systematic measurements of the inner source pickup ion fluxes as a function of radial distance between $0.28 \mathrm{au}$ and $1 \mathrm{au}$ and solar longitudes, a measurement of fundamental importance for testing existing models (Taut et al. 2015).

- Fast solar energetic electrons are excellent tracers of the magnetic topology and large-scale connectivity of the IMF in and out of the ecliptic plane (e.g. Malandraki et al. 2002, 2003, 2005; Tan et al. 2012; Gómez-Herrero et al. 2017). Near-relativistic solar energetic electron intensities and their anisotropies, as observed and provided by EPD, will be used as diagnostic tools of the large-scale structure and topology of the IMF threading through well-defined ICMEs in the inner heliosphere. Based on the time evolution of the angular distributions of the particle intensities as well as the time difference between the first outgoing and the first backstreaming electrons, the distance to existing magnetic mirrors backscattering the energetic electrons will be inferred. Moreover, the locations of the magnetic mirrors near the Sun or upstream from Solar Orbiter will be deduced.

- The temporal and spatial evolution of energetic particles accelerated by corotating interaction regions (CIRs) will be studied (e.g. Richardson 2004; Gómez-Herrero et al. 2011; Richardson 2018) by comparing EPD energetic particle measurements with relevant data from other missions in the heliosphere.

- The origin of relativistic ions in GLEs is still a topic of intense debate. There is evidence for a direct flare component (Masson et al. 2009; Kocharov et al. 2017) as well as a shock-accelerated component (Gopalswamy et al. 2012) in these events. The emission in well-connected GLEs usually starts right after the impulsive phase of the flare, which usually coincides quite well with the formation of the shock in the corona, marked by the commencement of a metric type II radio burst (Gopalswamy et al. 2012). Observations at the distance of the Earth are blurred by transport effects and so an accurate timing beyond an uncertainty of a few minutes in the release time is not possible. This hinders the association of particle release to the different flare and CME lift-off phenomena. In this respect, Solar Orbiter observations will provide a better timing of relativistic particle emission in the largest solar particle events and therefore it will improve our ability to relate a particle event to a particular phenomenon.

- In the ecliptic at $1 \mathrm{au}$, relativistic solar electrons have been demonstrated empirically to be an effective tool for predicting the intensity of slower, later arriving, deka-MeV solar energetic protons from the same SEP event (Malandraki \& Crosby 2018; Posner 2007). Currently, the physical relationship linking SEP electron and proton acceleration and transport processes near the Sun is not well understood. The comprehensive particle measurements to be provided by the EPD suite, in particular due to proximity to the Sun and reach to moderately high heliographic latitudes, have great potential to provide the necessary observational background to establish and/or limit such a causal relationship. Uncovering the physics behind the nature of this linkage could also increase understanding of space weather risks and enhance existing forecasting capabilities.

\section{Energetic Particle Detector suite overview}

\subsection{General description}

The EPD is an instrument suite comprising different sensors that have been designed to measure the spectra, composition, time variations, and directional distributions of energetic particles. These measurements will be performed over a partly overlapping energy range encompassing a few kiloelectronvolts to $450 \mathrm{MeV} \mathrm{n}^{-1}$, with sufficient time, energy, angular, and mass resolution to achieve the mission science goals. The EPD consists of the following units:

- SupraThermal Electrons and Protons (STEP).

- Electron Proton Telescope (EPT).

- Suprathermal Ion Spectrograph (SIS).

- High Energy Telescope (HET).

- Instrument Control Unit (ICU).

The EPT and HET sensors are located on two almost identical sensor units: EPT-HET1 and EPT-HET2. The ICU serves as a single power and data interface between the spacecraft and all the EPD units.The STEP, EPT, and HET were developed by the Christian Albrechts University (CAU) of Kiel. The SIS was developed by the Johns Hopkins University Applied Physics 

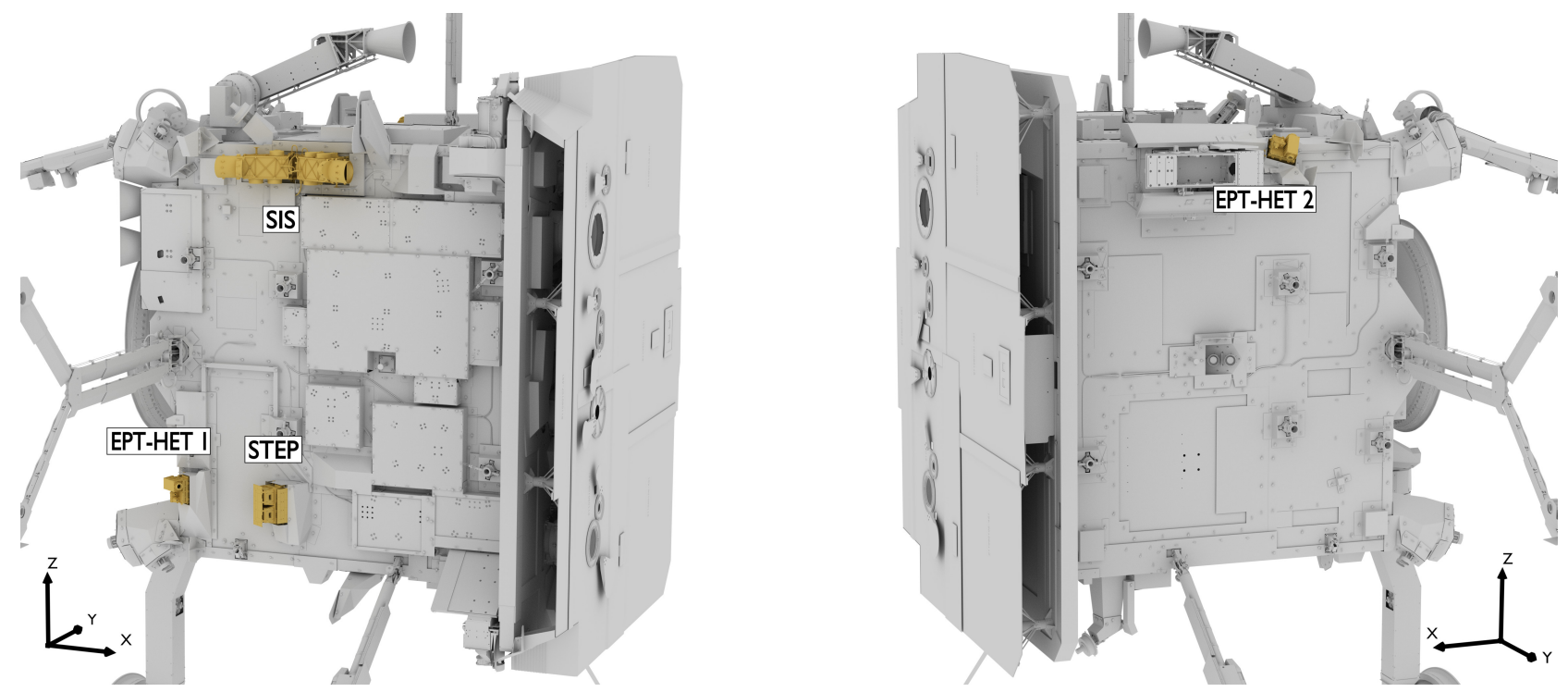

Fig. 1. Solar Orbiter spacecraft showing the location of the different EPD sensor units. In the coordinate system of this figure, $+X$ points towards the Sun and the $Y$ and $Z$ complete the orthogonal basis. The left side shows a view of the $-Y$ panel showing the sensors oriented along the mean Parker spiral direction, and the right side shows the view of the $+Y$ panel.

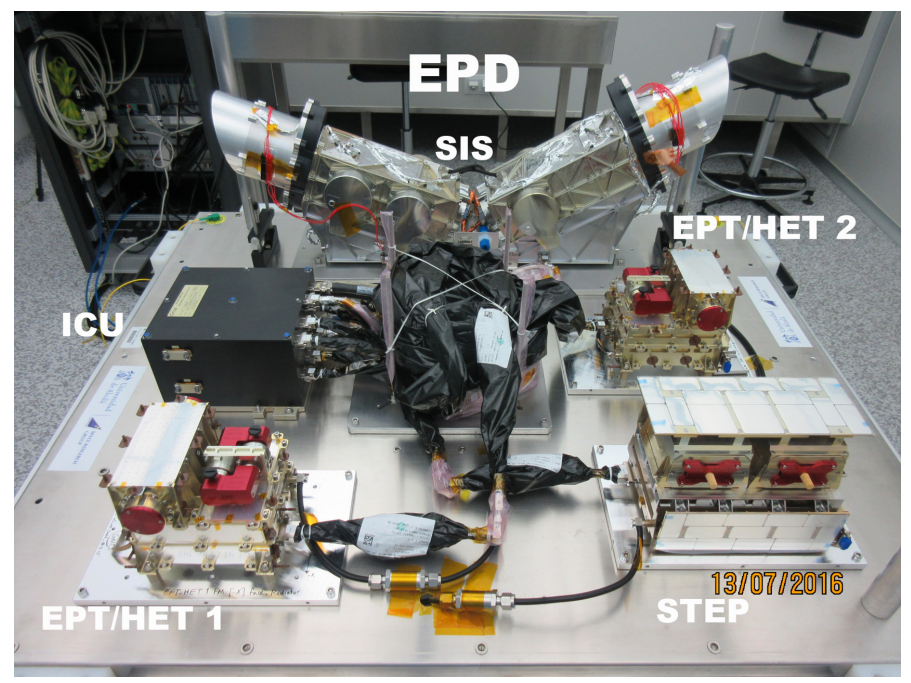

Fig. 2. EPD instrument suite during the integration tests performed in Madrid, July 2016. The picture shows the different EPD sensor units, the ICU, and the harness.

Laboratory (JHU/APL) and CAU. The ICU was developed by the ESA PI institution at the University of Alcalá, Spain. The location of the different EPD sensor units on the spacecraft is shown in Fig. 1.

All the units have significant heritage from previous missions. Their designs have been improved and optimised for the close approach to the Sun. As an example, the geometric factors are scaled to avoid saturation by high particle intensities close to the perihelia. Figure 2 shows a picture of the whole EPD instrument suite during the integration tests performed in July 2016.

The EPD energy range starts with suprathermal electrons $(E>2 \mathrm{keV})$ and extents up to low-energy galactic cosmic-ray $(\sim$ a few hundred of $\mathrm{MeV} \mathrm{n}^{-1}$ ), as shown in Fig. 3. This is achieved by a combination of the four EPD sensors which measure electrons, protons, and ions using different techniques.

Figure 4 shows the fields of view of the different EPD sensors in the spacecraft reference frame. The colour-coded background

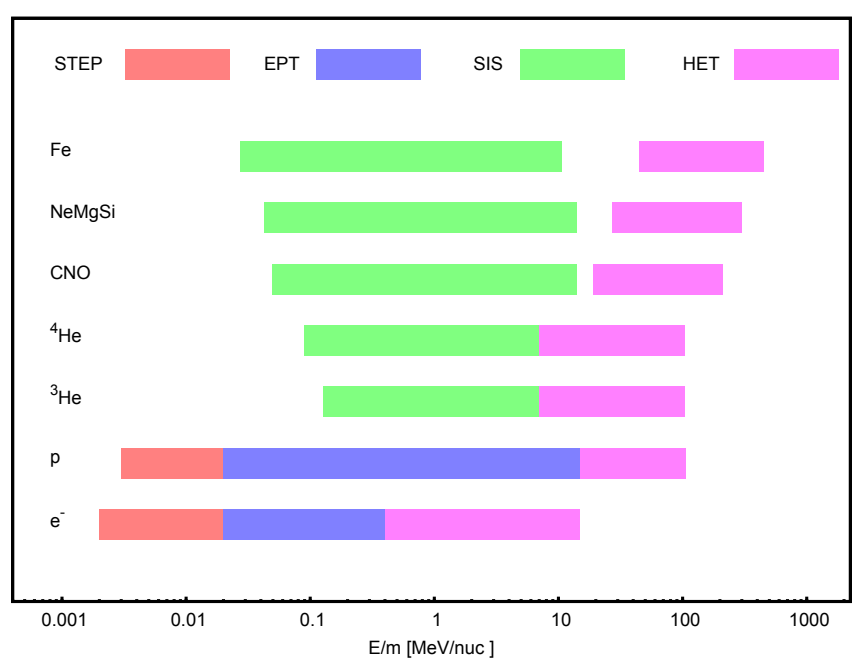

Fig. 3. Energy coverage of the EPD sensors for different species.

Solar Orbiter EPD fields of view

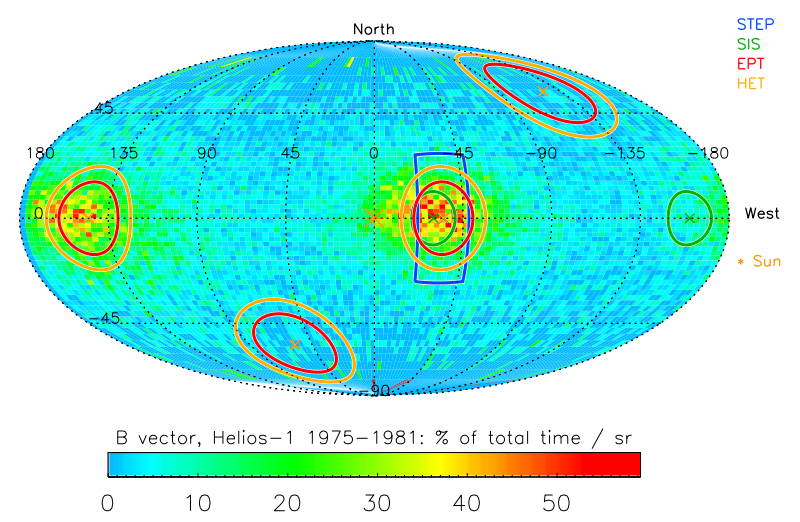

Fig. 4. Fields of view of the EPD sensors in the spacecraft reference frame. The colour-coded plot shows the hourly averaged magnetic-field distribution as observed by HELIOS-1. 
Table 1. Mass and power budget of the different EPD units.

\begin{tabular}{lcccccc}
\hline \hline Property & ICU & STEP & EPT-HET 1 & EPT-HET 2 & SIS & Total \\
\hline Mass $(\mathrm{g})$ & 2710 & 1998 & 1565 & 1566 & 6816 & $15718^{(a)}$ \\
Power $(\mathrm{W})^{(b)}$ & 4.0 & 2.9 & 4.4 & 4.6 & 3.8 & 19.7 \\
\hline
\end{tabular}

Notes. ${ }^{(a)}$ Including Intraharness and MLI. ${ }^{(b)}$ Beginning of life (BoL) in nominal mode.

corresponds to the hourly averaged magnetic field vector distribution as observed by HELIOS-1 during the years 1975-1981. HELIOS-1 had an in-ecliptic elliptical orbit with a perihelion at $0.31 \mathrm{au}$ and an aphelion at $0.99 \mathrm{au}$. We note the clustering of the sunward and anti-sunward apertures around the mean Parker spiral direction.

The mass and power budgets of EPD are given in Table 1. The overall nominal mass of the instrument is $15718 \mathrm{~g}$, which leads to a positive margin of $1282 \mathrm{~g}$ with respect to the spacecraft mass requirement. This mass is distributed over the five functional EPD units (STEP, SIS, EPT-HET1, EPT-HET2 and ICU), as well as their intraharness and multi-layer insulator (MLI). Regarding the power budget, EPD power average consumption in operational mode is roughly $19 \mathrm{~W}$, allowing for a wide safety margin of $8 \mathrm{~W}$ with respect to the allocated power budget for the EPD instrument. Each sensor unit of EPD requires a survival heater in order to keep it within its functional temperature range. The power consumption for the survival heaters is $16.36 \mathrm{~W}$, well below the allocated power of $17.7 \mathrm{~W}$.

\subsection{Data products}

The nominal telemetry budget of EPD is $3600 \mathrm{bps}$. Nevertheless, the elliptical orbit of Solar Orbiter will place the spacecraft at varying distances from the Earth, which will put restrictions on the instantaneous telemetry allocation available for the instruments. Although Solar Orbiter has a memory buffer to partly mitigate this effect, the EPD data production has to be planned in advance in order to maximise its scientific value. For this reason, EPD sensors have the ability to reduce their telemetry rates by changing the sampling cadences. Higher cadences will be used when the spacecraft is closer to the Sun. Another factor that will affect the instantaneous telemetry rate is solar activity, due to the adaptive compression scheme used by the units. This will require continuous adjustments of the observing plan in order to stay within the telemetry allocation.

EPD data can be classified in four categories:

- Housekeeping data

- Nominal science data

- Burst mode data

- Low latency data

All EPD sensors and the ICU will produce housekeeping data that will be received on Earth with the highest priority for monitoring instrument status and health.

Nominal science data will consist of count rates of energetic particles for selected energy intervals and species identified onboard by the different sensors. Table 2 gives a general view of EPD measurements. A detailed description of the data produced is given in the following sections, dedicated to the EPD sensors.

EPD sensors will be able, for short periods of time, to collect data with the highest cadence (up to 1 second for STEP, EPT, and HET and 3 seconds for SIS) and in some cases with higher energy resolution. This is the so-called burst mode. A burst mode period can be commanded from ground, but it can also be initiated by an autonomous onboard trigger. This trigger is handled by the ICU and can be configured during flight to detect interesting periods based on collected data. It can also respond to external triggers generated by other Solar Orbiter instruments via the Inter-Instrument Communication (IIC) service (see Walsh et al. 2020).

The latency of nominal data will depend on the particular position along the orbit and the state of the spacecraft memory buffer, meaning that for certain periods, the data will be received on Earth up to several months after it has been collected. Nevertheless, a small subset of scientific data will be sent to Earth with higher priority as low-latency data, typically the day after collection. The main purpose of these low-latency data is to give an overview of the recently acquired data, necessary for checking instrument performance, planning future operations, and to select periods of burst-mode data to be downlinked to Earth. Low-latency data products are detailed in Table 3. These products will be processed by an automated pipeline and be made available for all Solar Orbiter instrument teams as soon as they are received on Earth.

\subsection{Data processing on Earth}

Data produced on the spacecraft will be received on Earth in the form of CCSDS packets and distributed to the hosting institutions, where they will be processed and calibrated. All the data will be published to the scientific community 3 months after reception. Four different processing levels are considered:

- Level 0. Raw telemetry.

- Level 1. Uncalibrated data (i.e. counts).

- Level 2. Calibrated and validated data, ready for scientific use.

- Level 3. Higher processed products (e.g. pitch-angle distributions, browse plots, events lists, etc.).

Level 1 and level 2 products will be released in the form of daily files in Common Data Format (CDF), although other formats might be considered during the mission. All the data will be stored at the Solar Orbiter Archive (see Sanchez et al., in prep.) along with the data produced by all other Solar Orbiter instruments where they will be publicly accessible. Mirror repositories will also be located at the hosting institutions.

\section{The SupraThermal Electron Proton (STEP) sensor}

The STEP sensor (see Fig. 5) is designed to measure protons and electrons at supra-thermal energies (Table 2). For this purpose STEP employs principles and technologies used previously on other space-borne instruments. The basic detection principle is similar to that of the LION/COSTEP sensor on the Solar Heliospheric Observatory (SOHO; Müller-Mellin et al. 1995), the SEPT sensor on STEREO (Müller-Mellin et al. 2008), and the EPT sensor of EPD suite (Sect. 5). The STEP sensor employs two co-aligned sensor heads with a parallel field of view. One of the sensor heads contains a permanent magnet (see Sect. 4.2) to deflect electrons out of the nominal field of view. The sensor head with the magnet is called the "magnet channel" (MC) and will measure all particles in the nominal energy range except for electrons. The other sensor head will measure electrons in addition to ions, and is called the "integral channel" (IC). The difference of the count rates of the two sensor head detectors yields the electron count rate in the supra-thermal energy range. To measure the kinetic energy of the particles, each sensor head contains a solid state detector (SSD) with an ultra-thin window 
Table 2. Summary of key EPD measurement capabilities.

\begin{tabular}{|c|c|c|c|c|c|c|}
\hline & Species & Energy range & \# of FoVs & FoV size per aperture & $\begin{array}{l}\text { Geom. factor } \\
\left(\mathrm{cm}^{2} \mathrm{sr}\right)\end{array}$ & Max. time resolution \\
\hline STEP & $\mathrm{e}^{-}$, ions & $2-80 \mathrm{keV}$ & 1 (15 sectors) & $28^{\circ} \times 54^{\circ}$ & $8 \times 10^{-3(a)}$ & $1 \mathrm{~s}$ \\
\hline EPT & $\mathrm{e}^{-}, \mathrm{H}, \mathrm{He}$ & $\begin{array}{r}25-475 \mathrm{keV}\left(\mathrm{e}^{-}\right) \\
25 \mathrm{keV}-6.4 \mathrm{MeV}(\mathrm{H}) \\
1.6-6.4 \mathrm{MeV} \mathrm{n}^{-1}(\mathrm{He})\end{array}$ & $\begin{array}{r}4 \text { (sunward, } \\
\text { anti-sunward, } \\
\text { north, south) }\end{array}$ & $30^{\circ}$ & 0.01 & $1 \mathrm{~s}$ \\
\hline SIS & $\begin{array}{r}\mathrm{H},{ }^{3} \mathrm{He},{ }^{4} \mathrm{He}, \mathrm{C}, \mathrm{N}, \mathrm{O}, \\
\mathrm{Ne}, \mathrm{Mg}, \mathrm{Si}, \mathrm{S}, \mathrm{Ca}, \mathrm{Fe}\end{array}$ & $14 \mathrm{keV} / \mathrm{n}-20.5 \mathrm{MeV} \mathrm{n}^{-1}$ & $\begin{array}{r}2 \text { (sunward, } \\
\text { anti-sunward) }\end{array}$ & $22^{\circ}$ & $0.2^{(b)}$ & $3 \mathrm{~s}$ \\
\hline HET & $\begin{array}{r}\mathrm{e}^{-}, \mathrm{H},{ }^{3} \mathrm{He},{ }^{4} \mathrm{He}, \mathrm{C} \\
\mathrm{N}, \mathrm{O}, \mathrm{Ne}, \mathrm{Mg}, \mathrm{Si} \\
\mathrm{S}, \mathrm{Ar}, \mathrm{Ca}, \mathrm{Fe}, \mathrm{Ni}\end{array}$ & $\begin{array}{r}0.3-30 \mathrm{MeV}\left(\mathrm{e}^{-}\right) \\
6.8-107 \mathrm{MeV}(\mathrm{H}) \\
8.1-41 \mathrm{MeV} \mathrm{n}^{-1}\left({ }^{3} \mathrm{He}\right) \\
6.9-105 \mathrm{MeV} \mathrm{n}^{-1}\left({ }^{4} \mathrm{He}\right) \\
12-236 \mathrm{MeV} \mathrm{n}^{-1}(\mathrm{C}, \mathrm{N}, \mathrm{O}) \\
16-360 \mathrm{MeV} \mathrm{n}^{-1}(\mathrm{Ne}-\mathrm{S}) \\
20-500 \mathrm{MeV} \mathrm{n}^{-1}(\mathrm{Ar}-\mathrm{Ni})\end{array}$ & $\begin{array}{r}4 \text { (sunward, } \\
\text { anti-sunward, } \\
\text { north, south) }\end{array}$ & $43^{\circ}$ & $0.27^{(c)}$ & $1 \mathrm{~s}$ \\
\hline
\end{tabular}

Notes. ${ }^{(a)}$ Can be reduced to $1.7 \times 10^{-4} \mathrm{~cm}^{2}$ sr during high-intensity periods. ${ }^{(b)}$ Can be reduced in several steps to a minimum of $0.002 \mathrm{~cm}^{2} \mathrm{sr}$ using a variable aperture in front of the entrance foil. ${ }^{(c)}$ Can be reduced to $0.01 \mathrm{~cm}^{2} \mathrm{sr}$ during high-intensity periods.

and several pixels, which is based on technology used for STE on STEREO (Lin et al. 2008) (see Sect. 4.1). Angular resolution is achieved by using a small pinhole inside the sensor heads. The two sensor heads are mounted on a common electronics box, which itself is mounted on the $-y$-deck of the spacecraft. The sensor is mounted so that both fields of view point in the direction of the average nominal Parker spiral. Thus, the bore-sight directions are tilted by $35^{\circ}$ in the $x-y$ plane of the spacecraft towards the $-y$ axis (see Fig. 4).

\subsection{Solid state detectors}

For the detection of low-energy particles STEP relies on two identical silicon semiconductor diode detectors with ultra-thin entrance contacts (Tindall et al. 2008). The silicon diode detectors were fabricated using a $200 \AA$ thick phosphorous-doped polysilicon layer that forms the thin entrance window. A $220 \AA$ thick aluminum layer is deposited on top of the polysilicon in order to reduce the response to stray light.

Figure 6 illustrates the back side of the detector with its segmentation. The single pixels are arranged in an array of three rows and five columns. On top of that array there is a central segment/pixel for background determination. This pixel is shielded with an aluminum lid (the arm to hold that lid is partially visible in Fig. 7) on the entrance side to prevent particles from the nominal field of view from reaching the pixel. The pixel is therefore only susceptible to highly energetic particles penetrating the housing and passive parts of the sensor.

The detectors are operated fully depleted with a reverse bias voltage of about $+70 \mathrm{~V}$. Guard ring structures prevent leakage currents and cross-talk from one pixel to the others. To allow geometric factor adaptation the pattern of segments described above is repeated with a small offset using segments with a smaller area. The lengths of the segment edges are reduced from $2 \mathrm{~mm}$ for the large pixels to $0.3 \mathrm{~mm}$ for the smaller ones. The number of each small pixel corresponds to the number of the large pixel to the left of it in Fig. 6. Switching to the small pixels reduces the geometric factor by a factor of about 50, accordingly. The geometric factors are summarised in Table 2. Depending on the operational mode of the instrument, either all of the large or
Table 3. EPD low-latency data products.

\begin{tabular}{|c|c|c|c|c|}
\hline Species & Energy range & $\begin{array}{l}\text { \# of } \\
\text { energy } \\
\text { channels }\end{array}$ & $\begin{array}{l}\# \text { of } \\
\text { fields of } \\
\text { view }\end{array}$ & $\begin{array}{r}\text { Cadence } \\
\text { (s) }\end{array}$ \\
\hline \multicolumn{5}{|l|}{ STEP } \\
\hline Electrons & $2-80 \mathrm{keV}$ & 24 & 1 & 60 \\
\hline Electrons + Ions & $2-80 \mathrm{keV}$ & 24 & 1 & 60 \\
\hline Electrons & $2-60 \mathrm{keV}$ & 1 & 1 & 1 \\
\hline Electrons + Ions & $2-60 \mathrm{keV}$ & 1 & 1 & 1 \\
\hline \multicolumn{5}{|l|}{ EPT } \\
\hline Electrons & $25-400 \mathrm{keV}$ & 8 & 4 & 30 \\
\hline Protons & $25 \mathrm{keV}-6.4 \mathrm{MeV}$ & 16 & 4 & 30 \\
\hline Alpha particles & $6.4-25.6 \mathrm{MeV}$ & 2 & 4 & 30 \\
\hline Electrons & $50-100 \mathrm{keV}$ & 1 & 4 & 5 \\
\hline Protons & $50-200 \mathrm{keV}$ & 2 & 4 & 5 \\
\hline \multicolumn{5}{|l|}{ SIS } \\
\hline 12 species & $14 \mathrm{keV} \mathrm{n}^{-1}$ & $12 \times 21$ & 2 & 1800 \\
\hline from $\mathrm{H}$ to $\mathrm{Fe}$ & $-20.5 \mathrm{MeV} \mathrm{n}^{-1}$ & & & \\
\hline \multicolumn{5}{|l|}{ HET } \\
\hline Electrons & $0.3-30 \mathrm{MeV}$ & 4 & 4 & 30 \\
\hline Electrons & $0.8-11 \mathrm{MeV}$ & 1 & 4 & 5 \\
\hline Protons & $6.8-107 \mathrm{MeV}$ & 12 & 4 & 30 \\
\hline Protons & $13-107 \mathrm{MeV}$ & 3 & 4 & 5 \\
\hline Protons & $100-\infty \mathrm{MeV}$ & 6 & 4 & 30 \\
\hline${ }^{3} \mathrm{He}$ & $8.1-41 \mathrm{MeV} \mathrm{n}^{-1}$ & 10 & 4 & 300 \\
\hline${ }^{4} \mathrm{He}$ & $6.9-105 \mathrm{MeV} \mathrm{n}^{-1}$ & 14 & 4 & 300 \\
\hline${ }^{4} \mathrm{He}$ & $100-\infty \mathrm{MeV} \mathrm{n}^{-1}$ & 6 & 4 & 300 \\
\hline $\mathrm{C}, \mathrm{N}, \mathrm{O}$ & $13-236 \mathrm{MeV} \mathrm{n}^{-1}$ & $3 \times 5$ & 4 & 600 \\
\hline $\mathrm{C}, \mathrm{N}, \mathrm{O}$ & $200-\infty \mathrm{MeV} \mathrm{n}^{-1}$ & 6 & 4 & 3600 \\
\hline $\mathrm{Fe}$ & $23-33 \mathrm{MeV} \mathrm{n}^{-1}$ & 2 & 4 & 600 \\
\hline $\mathrm{Fe}$ & $82-630 \mathrm{MeV} \mathrm{n}^{-1}$ & 5 & 4 & 3600 \\
\hline
\end{tabular}

all of the small pixels are operated for data acquisition. We do not plan to operate both pixel sizes simultaneously.

The pixels are bond-wired to a state-of-the-art lownoise Application-Specific Integrated Circuit (ASIC), the IDeF-X BD (Gevin et al. 2015), to enable measurement of energy deposition in the low-kiloelectronvolt range. The input charges are converted into voltages by charge-sensitive amplifiers (CSAs). For noise-reduction purposes, the output signals of the 


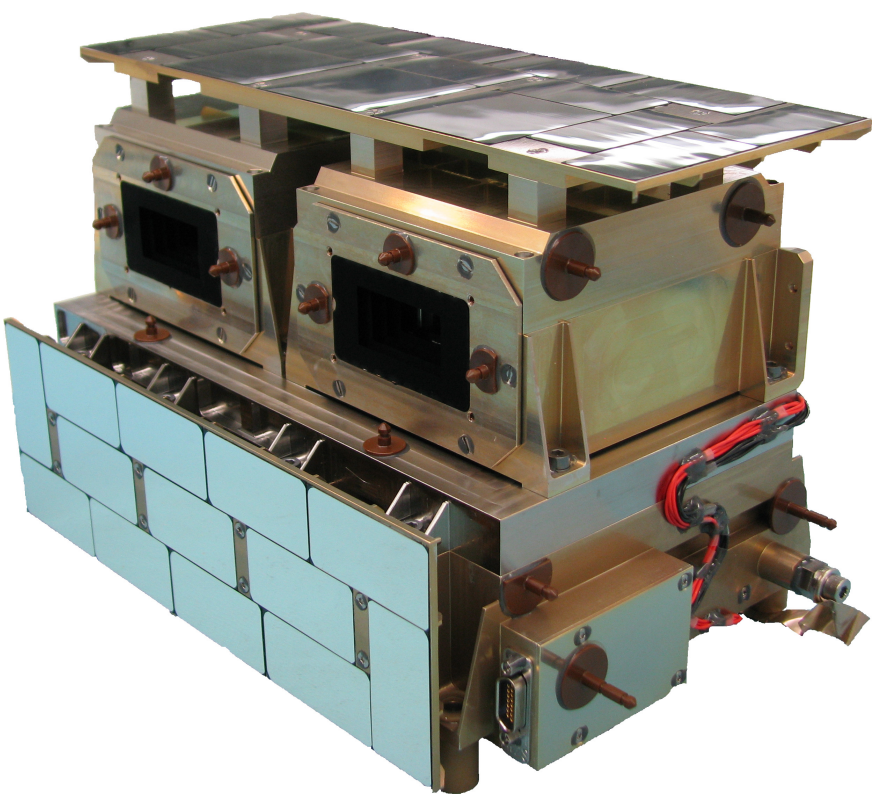

Fig. 5. STEP Flight Model of EPD without MLI. Two similar sensor heads are installed on a common electronics box (EBox). The sensor heads each contain a segmented SSD detector, an ASIC, a pinhole, and support structures. The right sensor head also includes the magnet assembly for electron deflection. The blackened apertures serve for stray-light reduction and passive cooling. In order to maintain the SSDs inside the sensor heads at a sufficiently low temperature, the EBox has a poor thermal coupling to the sensor heads. Optical Solar Reflectors (OSRs) are installed on top of the sensor heads and in the front of the EBox for heat emission purposes. The EBox contains all the other electronics required to operate and run the unit.

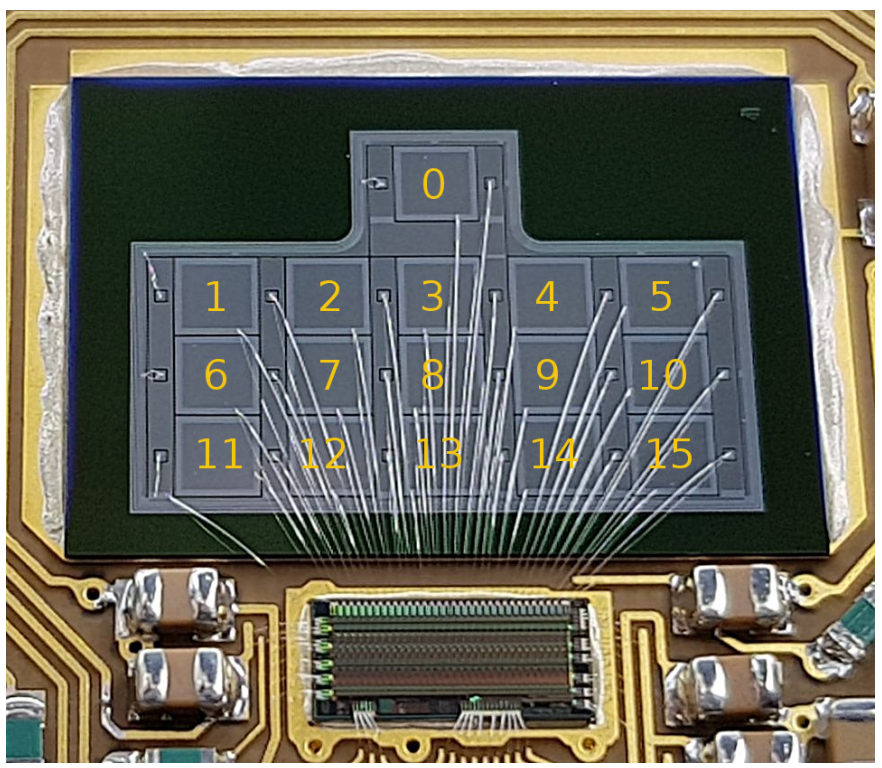

Fig. 6. Rear view of the segmented SSD detector. The segments are arranged in a $3 \times 5$ array. The large (numbered) segments are $2 \times 2 \mathrm{~mm}^{2}$ in dimension while the smaller pixels (same pattern shifted rightwards when seen from behind) are $0.3 \times 0.3 \mathrm{~mm}^{2}$ in size. The central uppermost segment (\#0) is for GCR background determination. The numbers are added to the photo to explain the pixel numbering. For incoming particles the pixel numbering appears mirrored along the central column, accordingly.

CSAs are then shaped by filters with programmable filtering time constants. The settings of the ASIC are fully flight-configurable.


Fig. 7. Assembly of the STEP Magnet Channel detector with (left) and without $(r i g h t)$ front pinhole.

Superposition of $5 \mathrm{keV}$ proton FOVs (magnet and interal channels, side view)
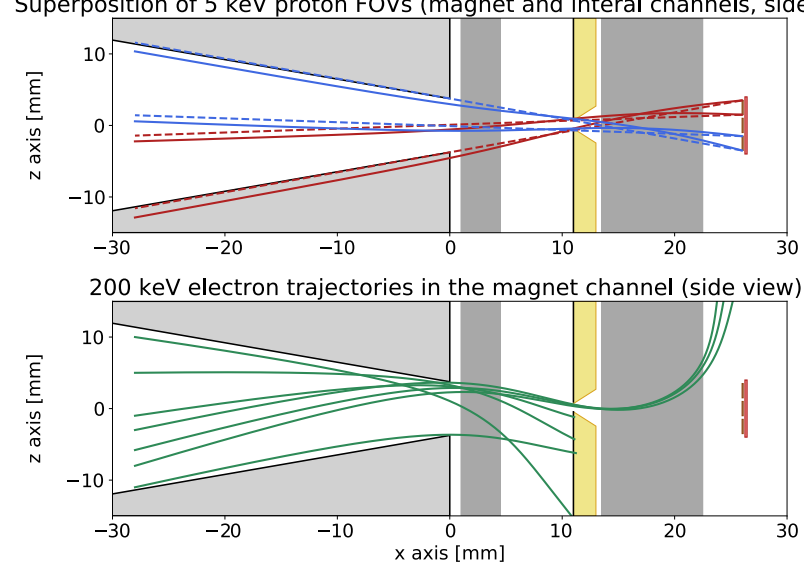

Fig. 8. Top: comparison of the calculated FoVs (side view) of the STEP MC (solid line) and IC (dashed line). Bottom: side view of example $200 \mathrm{keV}$ electron trajectories. The apertures are schematically sketched in light grey, the magnets in a darker grey. The pinhole has a beige coloration while the segmented detector is coloured reddish. We note that the figure is true to scale. A slight asymmetry of ion trajectories in the magnet head is taken into account by shifting the corresponding magnet-head pinhole by $0.25 \mathrm{~mm}$ in $+z$ direction.

The duty cycle of the SSD itself is $100 \%$. For an assessment of the duty cycle of the whole sensor, the limitations/contributions of the connected read-out electronics need to be taken into account. With the current timing settings about $10 \mu \mathrm{s}$ is required to detect, shape, digitise, and store a particle hit in one of the pixels of the SSD.

\subsection{Particle separation and geometrical properties}

The particle trajectories inside the sensor heads are influenced by multiple optical elements. As mentioned earlier, the basic working principle of both sensor heads is based on a segmented SSD together with a pinhole to achieve angular resolution. In the IC no further optical elements are present and the concept of geometric optics applies (see upper part of Fig. 8, dashed lines). The blackened front apertures for straylight reduction are aligned with the resulting FoVs.

In contrast to the IC, the MC includes a fine-tuned magnetassembly in order to filter electrons with energies lower than $200 \mathrm{keV}$ while virtually not affecting the ion trajectories. Figure 9 shows a computer-aided-design (CAD) view of the optically relevant elements inside the MC. The pinhole and the detector are located at the same position along the particle 


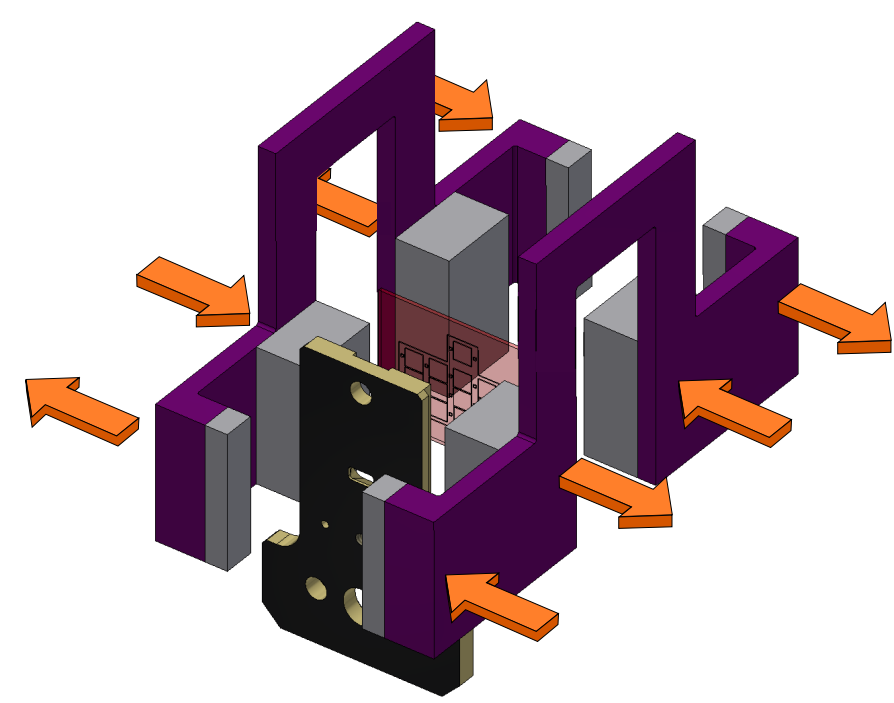

Fig. 9. CAD view of the elements with an influence on the particle trajectories of the STEP MC: the segmented detector is coloured reddish The pinhole structure is black/beige. The magnets are illustrated in grey while the yokes of the magnets are purple. The orientation of each magnetic field is indicated with orange arrows. The front apertures are not shown. The structure of the IC is similar, but without the magnets and their yokes.

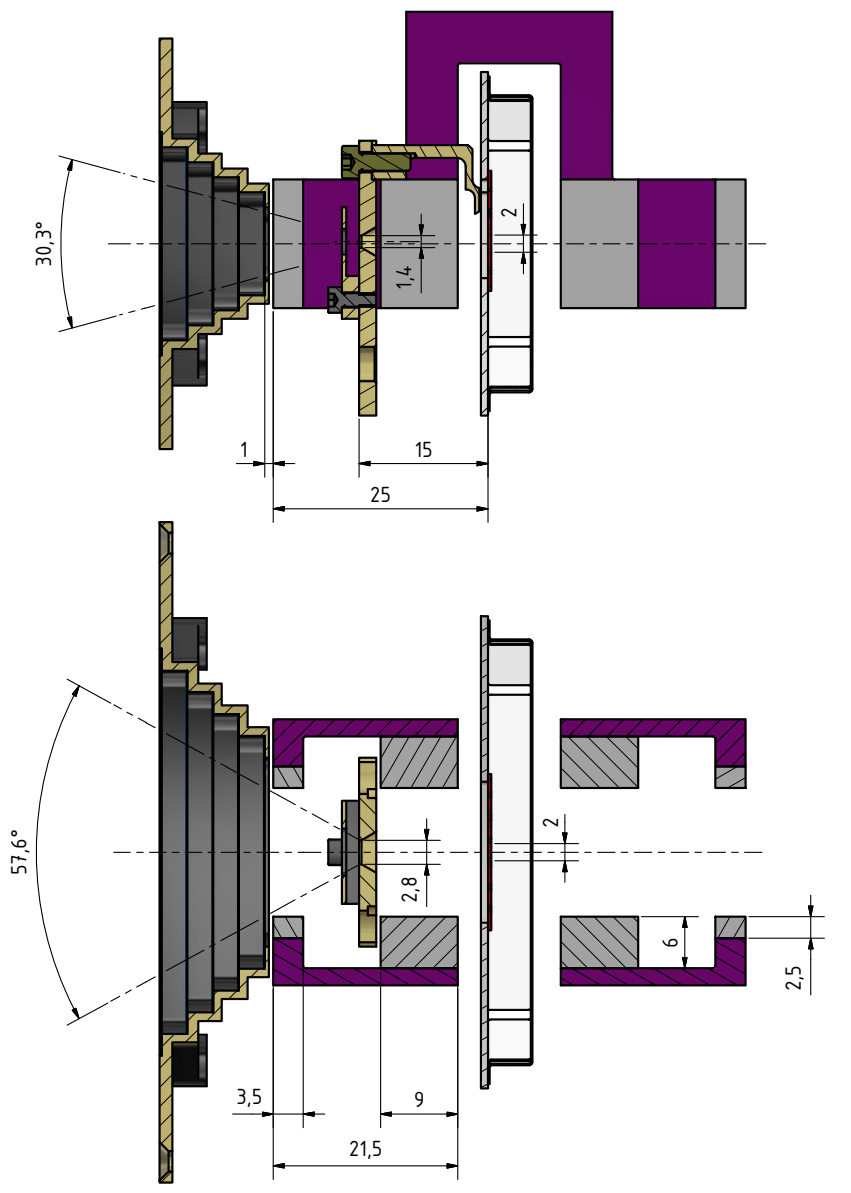

Fig. 10. CAD view of the optically relevant items of the MAGNET channel sensor head. Top: side view. Bottom: top view. Details of the geometries in Fig. 8 are shown. In the INTEGRAL channel the magnets are not present and the pinhole is installed $0.25 \mathrm{~mm}$ lower. The colour scheme is the same as in Figs. 8 and 9.

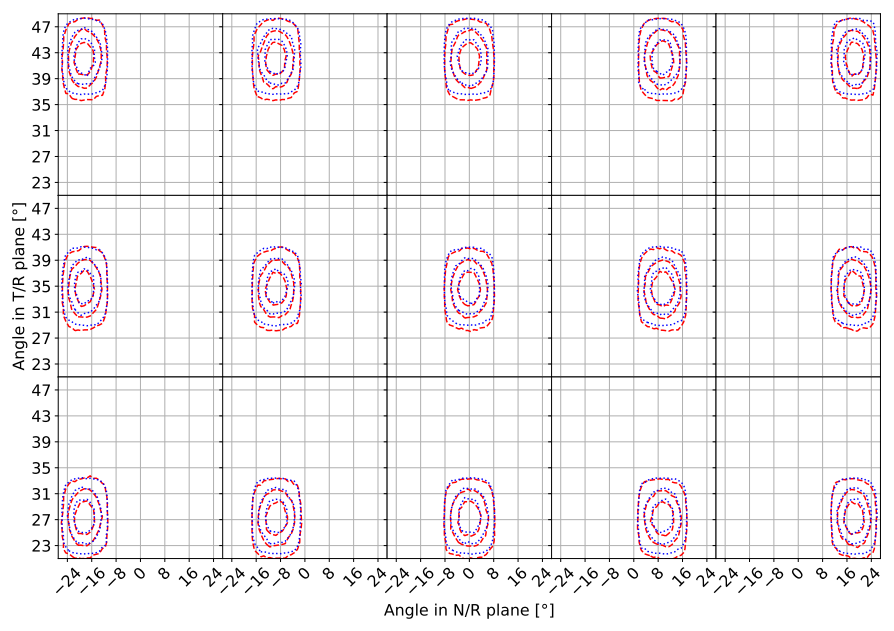

Fig. 11. Simulated angular response of the individual STEP pixels for integral (blue, dotted) and magnet channel (red, dashed). The contour levels are $66 \%, 33 \%$, and $5 \%$.

trajectories ( $x$-axis). The pinhole of the MC has a tiny offset of $+0.25 \mathrm{~mm}$ in the $z$-axis of the instrument (axis as per Fig. 8). A detailed sketch of the STEP MC sensor head geometry is shown in Fig. 10.

The first set of permanent magnets installed in front of the pinhole bends the electrons (with energies below $200 \mathrm{keV}$ ) sufficiently downwards preventing them from passing the pinhole. In some rare cases (when incoming direction, offset, and energy match) the electrons can nevertheless pass the pinhole. In these rare cases a second set of stronger (larger) permanent magnets installed between the pinhole and the detector then reliably sweeps these electrons in the opposite direction not allowing them to reach the detector. With this technique electrons in the relevant energy range are prevented from directly interacting with the SSD. At the same time, the magnet assembly needs to ensure a virtually unaffected passage of ions of all relevant species and energies (including the least-rigid $4 \mathrm{keV}$ protons) through the sensor head up to the SSD. This feature is necessary to allow differential measurements between both sensor heads. In fact, the ion trajectories are altered when traversing through the magnetic fields. While the first set of magnets bends the ions in the $+z$-direction (see Fig. 8), the second (stronger) set of magnets over-focuses the ion trajectories back in the $-z$-direction. The plane of intersection of the over-focused and the non-altered trajectories lies in the plane of the SSD detector. Thus, ions with identical parameters (ion species, energy, direction, and offset) will be detected in the same segment of the SSD with identical energy no matter which sensor head they are passing. In the $y$-direction no forces act on the particles.

The upper part of Fig. 8 shows the resulting FoVs for both sensor heads. It shall be pointed out that the FoV of the MC has a minimal offset compared to the FoV of the IC in the $z$-axis. This requires calibration and needs to be taken into account when analysing the acquired data. The opening angle and direction of both FoVs match very well. The lower part of that figure shows example electron trajectories in the MC demonstrating the good electron screening capabilities of that system. The angular response for ions at each segment of both sensor heads is shown in Fig. 11. The coincidence of the FoVs allows for the use of differential measurements between both channels.

In order to comply with the stringent mission requirements regarding electromagnetic compatibility (EMC) the 
above-described magnetic assembly consisting of two sets of magnet pairs of different magnetic strengths was doubled by adding two identical pairs with contrary orientation, all combined via two yokes (see Fig. 9). This approach turns the dipolar magnetic fields into quadrupolar ones with a considerably faster decline.

\subsection{Calibration of the STEP unit}

The STEP unit employs a total of 64 SSD segments that are separately connected to the corresponding channels of one of the two ASICs. For calibration purposes the STEP unit was operated in a thermal vacuum chamber with a mission-plausible thermal gradient, acquiring ${ }^{133} \mathrm{Ba}$ data and specifically focusing on the lowenergy gamma lines. A mission-expected thermal gradient was established in the unit at multiple mission-plausible temperatures. The calibration of each channel was found to vary with temperature. With the acquired data, a linear dependency of the calibration parameters on the IX (=ASIC) temperature was observed. The calibration parameters are explicitly given in Table 4 . The intrinsic energy resolution of each calibrated channel was measured to be better than $750 \mathrm{eV}$ FWHM even at room temperature, and a mean sensitivity of less than $2 \mathrm{keV}$ was achieved.

Figure 12 shows calibrated ${ }^{133} \mathrm{Ba}$ spectra for the two sensor heads integrated over all 32 channels of the particular sensor head and over several temperatures (ranging from $+22^{\circ} \mathrm{C}$ to $-52^{\circ} \mathrm{C}$ Idef$\mathrm{X}$ temperature). These measurements are used to verify the validity of the thermal calibrations of the various channels.

The FWHMs of the $53 \mathrm{keV}$ line of both integrated spectra are around 700 for the IC and $800 \mathrm{eV}$ for the MC. The additional line in the MC at roughly $37-39 \mathrm{keV}$ stems from the fluorescence of $\mathrm{Nd}$ (Neodymium), which has been used in the permanent magnets inside the MC sensor head.

The in-flight calibration of STEP will be possible using binned count rates around $8 \mathrm{keV}$ as the copper covers surrounding the sensor head electronics in both sensor heads emit characteristic secondary X-rays at that energy ( $8 \mathrm{keV}$ copper fluorescence). This peak will be used as a calibration line and will allow for the calibration factors to be monitored and refined throughout the mission. For data analysis the energy loss in the $300 \AA$ aluminium layer for stray-light reduction needs to be taken into account. Additionally, the ASIC (Sect. 4.1) offers the possibility to inject test pulses, which can be used for in-flight calibration as well.

The energy measurement of STEP is temperature dependent, meaning that the offset of the analog-to-digital converters (ADC) readout of the digitised energy deposition varies with temperature. The dependency is linear, and a calibration has been performed for both the Flight Model (FM) and Flight Spare (FS) during thermal vacuum testing. A selection of the calibration results is shown in Fig. 13. The gain of the energy to ADC conversion has been found to be independent of temperature.

The geometry response of both sensor heads was determined using Geant4 simulations (Agostinelli et al. 2003), and are tabulated in Table 4. The response to electrons is slightly enhanced in the IC compared to protons due to electron scattering at the aperture of the telescope, thus slightly increasing the angular acceptance for electrons.

\subsection{Data processing in the sensor}

\subsubsection{Level-1 and level-2 trigger logic of STEP}

When a particle trigger occurs in one or multiple detector segments, the Idef-X ASIC will provide the trigger mask, describing which of the pixels got hit, and the energy loss in the hit segments.

For single particle interactions limited to one active segment of the SSD, the energy loss of the particle can be determined correctly. In cases where multiple active SSD segments are hit by incoming particles within the peaking time (flight-configurable, $6 \mu \mathrm{s}$ at time of launch), only the shaped signal of the current pulse that was the first to be induced will reach its full maximum peak in the shaper with certainty, before all signals go into peak-hold, which occurs when the field-programmable gate array (FPGA) issues the read-out signal to the ASIC. All current pulses that did not fully peak during the shaping time will not have reached their maximum, and thus their determined energy levels will be systematically too low. Since it is impossible to reproduce the chronological order of the arrival of the pulses, it is impossible to determine which of the channels has seen the full signal, and which has not.

The trigger mask is the level-1 trigger of STEP. The FPGA investigates the coincidence condition based on the trigger mask, that is, which combination of SSD pixels have been hit. The coincidences between the pixels control which of the four hardcoded level-2 triggers is triggered. Each level-2 trigger is associated with one of four level-3 trigger classes. Table 5 lists the level-2 and corresponding level-3 trigger classes of STEP.

\subsubsection{Level-3 trigger STEP}

The level-3 trigger engine is common to the EPT (Sect. 5), HET (Sect. 7), and STEP sensors, and is a fully flight-configurable assembly-like instruction set implemented in the digital board FPGA. For each trigger class, up to 256 instructions can be processed. The result of the level-3 trigger is usually the increment of one or more histogram bins in the histogram memory, as well as controlling the filling of the 16 Pulse-Height-Analysis (PHA) buffers. The definitions of the PHA buffers used for STEP at the date of launch of the mission are shown in Table 6.

\subsubsection{Data products}

The data product engine is common to the EPT (Sect. 5), HET (Sect. 7), and STEP sensors, and uses a fully flight-configurable configuration table with up to 2048 entries corresponding to individual data products. The entries control the creation of science data telemetry to the ICU (Sect. 8). Entries are evaluated at a configurable cadence (usually $1 \mathrm{~s}$ ) by the sensor FPGA. A single entry represents the sum of one or more bins in the histogram memory of the sensor, meaning that the data products can be used to represent an integration of multiple histogram bins. For each entry, the summing cadence and the number representation of the integration can be configured. The cadence for each entry can be set to values between a minimum of one second and maximum cadence of up to one hour. The number representation can be selected as well, to trade off telemetry volume and precision. For each entry, it is additionally possible to use a compression algorithm that takes into account the Poissonian nature of the counting process. The compression will slightly increase the error associated with a transmitted counter, but is designed to decrease the data rate if the changes in the count rate are low during one summing cadence. This is achieved by using runlength compression in addition to dropping bits deemed insignificant with respect to the Poissonian statistics. The compression engine records the error during one cycle, so after the "cycle cadence", the compression engine will transmit the "missed" counts due to the error propagation. This means that after a full 
Table 4. Overview of STEP FS key parameters.

\begin{tabular}{|c|c|c|c|c|c|c|c|}
\hline $\begin{array}{l}\text { SSD } \\
\text { pixel }\end{array}$ & $\begin{array}{l}\text { Proton resp. } \\
\left(10^{-3} \mathrm{~mm}^{2} \mathrm{sr}\right)\end{array}$ & $\begin{array}{l}\text { Electron resp. } \\
\left(10^{-3} \mathrm{~mm}^{2} \mathrm{sr}\right)\end{array}$ & $\begin{array}{l}\mathrm{cp}_{1} \\
\left(10^{-3} \mathrm{eV} / \mathrm{ADC}\right)\end{array}$ & $\begin{array}{l}\mathrm{cp}_{2} \\
\left(\mathrm{ADC} /{ }^{\circ} \mathrm{C}\right)\end{array}$ & $\begin{array}{l}\mathrm{cp}_{3} \\
(\mathrm{ADC})\end{array}$ & $\begin{array}{l}\langle F W H M\rangle \\
(\mathrm{eV})\end{array}$ & $\begin{array}{l}\text { Sensitivity } \\
(\mathrm{keV})\end{array}$ \\
\hline \multicolumn{8}{|c|}{ Integral channel large pixels } \\
\hline 0 & 0 & 0 & 37.1 & 0.953 & 2942 & 711 & 2.4 \\
\hline 1 & 47 & 49 & 38.1 & 1.318 & 2791 & 669 & 2.5 \\
\hline 2 & 56 & 57 & 36.6 & 0.822 & 2956 & 702 & 2.6 \\
\hline 3 & 59 & 59 & 37.8 & 0.350 & 2976 & 649 & 2.4 \\
\hline 4 & 56 & 57 & 37.0 & 0.968 & 2939 & 672 & 2.4 \\
\hline 5 & 47 & 48 & 36.9 & 0.495 & 2979 & 708 & 2.4 \\
\hline 6 & 49 & 50 & 37.2 & 0.816 & 2970 & 736 & 2.3 \\
\hline 7 & 57 & 59 & 36.8 & 1.188 & 2886 & 676 & 2.5 \\
\hline 8 & 61 & 62 & 36.8 & 1.260 & 2952 & 728 & 2.4 \\
\hline 9 & 57 & 59 & 37.3 & 0.502 & 3051 & 656 & 2.1 \\
\hline 10 & 49 & 50 & 37.2 & 0.767 & 3043 & 698 & 2.3 \\
\hline 11 & 48 & 49 & 37.3 & 0.642 & 2815 & 671 & 2.4 \\
\hline 12 & 55 & 56 & 37.3 & 1.241 & 2925 & 671 & 2.2 \\
\hline 13 & 58 & 60 & 36.4 & 1.286 & 2853 & 691 & 2.4 \\
\hline 14 & 55 & 56 & 36.7 & 1.014 & 2972 & 667 & 2.7 \\
\hline 15 & 48 & 49 & 38.0 & 0.981 & 2980 & 660 & 2.2 \\
\hline \multicolumn{8}{|c|}{ Integral channel small pixels } \\
\hline 0 & 0 & 0 & 36.6 & 0.723 & 2949 & 658 & 2.0 \\
\hline 1 & 1.2 & 1.4 & 36.6 & 0.670 & 3032 & 629 & 1.9 \\
\hline 2 & 1.3 & 1.4 & 36.3 & 0.743 & 2929 & 631 & 1.7 \\
\hline 3 & 1.3 & 1.6 & 37.1 & 1.030 & 2978 & 622 & 1.8 \\
\hline 4 & 1.1 & 1.3 & 36.4 & 0.891 & 3046 & 589 & 1.9 \\
\hline 5 & 0.9 & 1.1 & 38.2 & 1.067 & 2890 & 656 & 1.9 \\
\hline 6 & 1.2 & 1.4 & 36.1 & 0.771 & 2932 & 627 & 1.7 \\
\hline 7 & 1.3 & 1.5 & 37.9 & 1.329 & 2941 & 689 & 1.8 \\
\hline 8 & 1.4 & 1.5 & 36.5 & 0.754 & 3012 & 627 & 1.9 \\
\hline 9 & 1.2 & 1.4 & 37.0 & 0.864 & 2936 & 576 & 1.8 \\
\hline 10 & 0.9 & 1.1 & 37.3 & 0.695 & 2942 & 596 & 1.9 \\
\hline 11 & 1.2 & 1.2 & 37.3 & 0.850 & 2958 & 581 & 1.7 \\
\hline 12 & 1.3 & 1.5 & 36.9 & 0.752 & 2887 & 612 & 2.0 \\
\hline 13 & 1.3 & 1.5 & 37.1 & 1.286 & 2894 & 615 & 1.6 \\
\hline 14 & 1.2 & 1.4 & 36.9 & 0.879 & 2899 & 545 & 2.0 \\
\hline 15 & 1.0 & 1.1 & 37.1 & 0.982 & 2979 & 645 & 1.6 \\
\hline \multicolumn{8}{|c|}{ Magnet channel large pixels } \\
\hline 0 & 0 & 0 & 37.8 & 1.387 & 2834 & 695 & 2.3 \\
\hline 1 & 53 & 0 & 37.5 & 1.264 & 2899 & 659 & 2.3 \\
\hline 2 & 62 & 0 & 38.5 & 0.952 & 2860 & 654 & 2.4 \\
\hline 3 & 66 & 0 & 38.2 & 0.458 & 3020 & 656 & 2.6 \\
\hline 4 & 63 & 0 & 38.2 & 0.721 & 2939 & 687 & 2.4 \\
\hline 5 & 53 & 0 & 37.5 & 0.678 & 3041 & 681 & 2.4 \\
\hline 6 & 54 & 0 & 38.5 & 0.956 & 2899 & 669 & 2.2 \\
\hline 7 & 64 & 0 & 37.8 & 1.269 & 3020 & 680 & 2.4 \\
\hline 8 & 68 & 0 & 38.3 & 1.399 & 2797 & 666 & 2.3 \\
\hline 9 & 64 & 0 & 37.5 & 0.696 & 2988 & 674 & 2.4 \\
\hline 10 & 53 & 0 & 38.3 & 0.973 & 2817 & 658 & 2.4 \\
\hline 11 & 51 & 0 & 38.0 & 0.918 & 2928 & 691 & 2.6 \\
\hline 12 & 61 & 0 & 37.6 & 1.156 & 2898 & 676 & 2.0 \\
\hline 13 & 65 & 0 & 38.0 & 1.245 & 2950 & 683 & 2.4 \\
\hline 14 & 61 & 0 & 38.6 & 0.663 & 2914 & 686 & 2.5 \\
\hline 15 & 51 & 0 & 38.1 & 1.149 & 2850 & 669 & 2.1 \\
\hline
\end{tabular}

Notes. The pixel numbering follows Fig. 6. The geometric response factors are listed in the second and third columns. They apply to protons and electrons with energies starting from $10 \mathrm{keV}$ respectively. The temperature-correlated calibration parameters $\mathrm{cp}_{i}$ for each channel are given in the Cols. 4-6. To determine the detected energy $E$ in eV from the Analog-to-Digital-Converters (ADC) reading at a given IX-temperature $T$, the following formula has to be applied: $E[\mathrm{eV}]=\left(\mathrm{cp}_{2} \times T+\mathrm{cp}_{3}+\mathrm{ADC}\right) \times \mathrm{cp}_{1}$ with $T$ in ${ }^{\circ} \mathrm{C}$. These parameters have been derived from multiple ${ }^{133} \mathrm{Ba}$ calibration measurements across the whole mission-expected temperature range maintaining the expected thermal gradient throughout the unit. The mean FWHMs stated in the seventh column have been derived from the $81 \mathrm{keV}$ peaks of the ${ }^{133} \mathrm{Ba}$ calibration data at multiple temperatures. The sensitivity describes the least sensitive performance of each particular channel, again taking all the aforementioned calibration data at different temperatures into account. 
Table 4. continued.

\begin{tabular}{llllllll}
\hline \hline $\begin{array}{l}\text { SSD } \\
\text { pixel }\end{array}$ & $\begin{array}{l}\text { Proton resp. } \\
\left(10^{-3} \mathrm{~mm}^{2} \mathrm{sr}\right)\end{array}$ & $\begin{array}{l}\text { Electron resp. } \\
\left(10^{-3} \mathrm{~mm}^{2} \mathrm{sr}\right)\end{array}$ & $\begin{array}{l}\mathrm{cp}_{1} \\
\left(10^{-3} \mathrm{eV} / \mathrm{ADC}\right)\end{array}$ & $\begin{array}{l}\mathrm{cp}_{2} \\
\left(\mathrm{ADC} /{ }^{\circ} \mathrm{C}\right)\end{array}$ & $\begin{array}{l}\mathrm{cp}_{3} \\
(\mathrm{ADC})\end{array}$ & $\begin{array}{l}\langle F W H M\rangle \\
(\mathrm{eV})\end{array}$ & $\begin{array}{l}\text { Sensitivity } \\
(\mathrm{keV})\end{array}$ \\
\hline \multicolumn{2}{l}{ Magnet channel small pixels } & & & & & \\
\hline 0 & 0.0 & 0 & 36.5 & 0.333 & 2989 & 620 & 2.0 \\
1 & 1.3 & 0 & 37.8 & 0.684 & 2989 & 604 & 1.9 \\
2 & 1.4 & 0 & 37.8 & 1.115 & 2908 & 626 & 1.7 \\
3 & 1.5 & 0 & 37.2 & 0.730 & 2962 & 602 & 1.9 \\
4 & 1.3 & 0 & 38.0 & 1.318 & 2842 & 638 & 1.8 \\
5 & 1.1 & 0 & 37.6 & 0.766 & 2999 & 601 & 2.1 \\
6 & 1.3 & 0 & 37.0 & 1.084 & 2838 & 606 & 1.8 \\
7 & 1.5 & 0 & 37.9 & 0.890 & 2919 & 621 & 1.9 \\
8 & 1.5 & 0 & 38.2 & 1.138 & 3018 & 610 & 1.9 \\
9 & 1.3 & 0 & 36.9 & 1.005 & 2942 & 590 & 1.9 \\
10 & 1.1 & 0 & 38.5 & 0.931 & 2906 & 608 & 2.0 \\
11 & 1.4 & 0 & 37.3 & 0.921 & 2913 & 611 & 1.7 \\
12 & 1.4 & 0 & 38.1 & 0.771 & 2980 & 586 & 1.8 \\
13 & 1.4 & 0 & 38.2 & 1.608 & 2893 & 580 & 1.9 \\
14 & 1.3 & 0 & 38.4 & 0.727 & 3079 & 606 & 1.7 \\
15 & 1.0 & 0 & 37.7 & 1.045 & 3026 & 640 & 2.2 \\
\hline
\end{tabular}

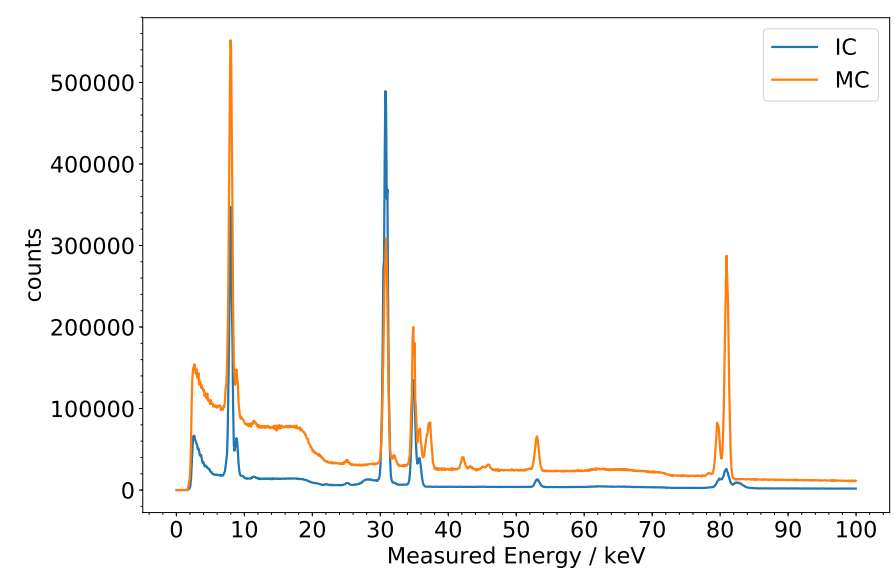

Fig. 12. Spectra of ${ }^{133} \mathrm{Ba}$ acquired with STEP FS for the magnet (orange) and integral (blue) channels. Spectra are integrated over all pixels and for several temperatures.

cycle, no counts are lost and the count rate is accurately represented. Thus, the compression algorithm allows for sudden changes in count rate to be recorded (albeit with larger error) even at shorter cadences than the cycle cadence.

Table 7 shows the data products defined for STEP at the time of launch. All data products are the same for the integral channel and magnet channel, allowing for the usage of the subtraction method to obtain the electron count rate. For the numbering of the pixels of the individual data products, please refer to Fig. 6.

The Nominal data products come in two separate configurations, "close orbit" and "far orbit". Compared to "far orbit", the "close orbit" configuration offers higher cadences, and this mode is intended to be used when the spacecraft is close to the Sun. These modes are stored as separate configurations, and can be switched via telecommanding (Sect. 4.5).

\subsection{Operating modes}

The STEP operations are controlled using a fully flightconfigurable table. Up to 20 different configurations can be

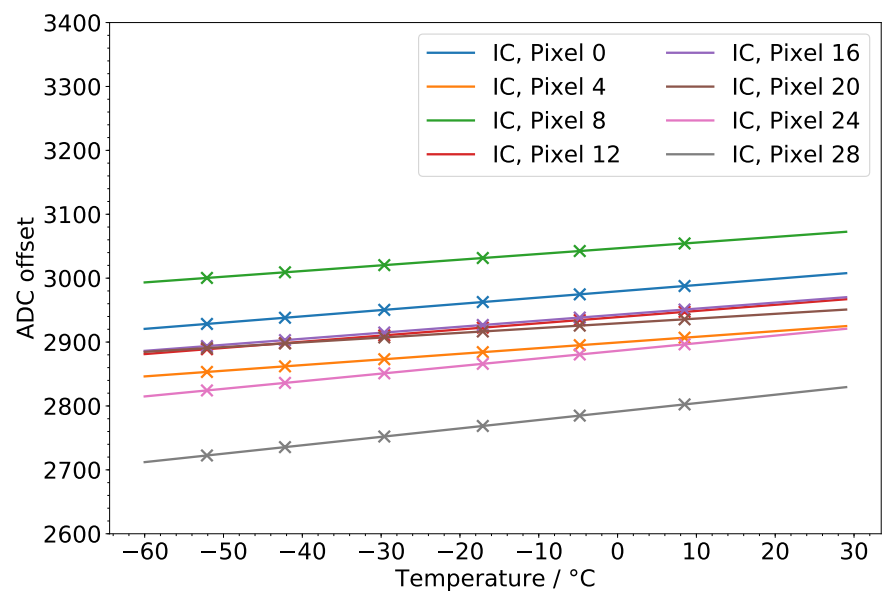

Fig. 13. Dependence of the ADC offset on the ASIC temperature of the STEP FS unit for a selection of pixels.

stored in the table to control the sensor. The operational modes outlined below are based on the configuration of the STEP sensor at the time of launch. These modes are the following:

- Off: The sensor is switched off, and does not consume power or generate data.

- Idle: The sensor is switched on, but is unconfigured and does not generate any data.

- Housekeeping: The sensor is configured to record and telemeter housekeeping data.

- Baseline Operational mode - Low flux, far orbit: The sensor is configured to record and telemeter housekeeping data, as well as scientific data for the large pixels.

- Operational mode - High flux, far orbit: The sensor is configured to record and telemeter housekeeping data, as well as scientific data for the small pixels.

- Operational mode - Low flux, close orbit: The sensor is configured to record and telemeter housekeeping data, as well as scientific data for the large pixels at a higher cadence.

- Operational mode - High flux, close orbit: The sensor is configured to record and telemeter housekeeping data, as well as scientific data for the small pixels at a higher cadence. 
Table 5. STEP Level-2 trigger logic.

\begin{tabular}{lccl}
\hline \hline L2 trigger \# & Coincidence condition & L3 trigger class & Description \\
\hline 0 & $p_{0, i} \wedge \overline{\sum_{j \neq i} p_{0, j}}$ & 0 & Single pixel hit IC \\
1 & $p_{1, i} \wedge \overline{\sum_{j \neq i} p_{1, j}}$ & 1 & Single pixel hit MC \\
2 & $p_{0, i} \wedge p_{0, j \neq i}$ & 2 & Multiple pixels hit IC \\
3 & $p_{1, i} \wedge p_{1, j \neq i}$ & 3 & Multiple pixels hit MC \\
\hline
\end{tabular}

Notes. $p_{n, i}$ denotes the $i$ th pixel of the $n$th SSD. The sum symbol $\sum$ denotes a chain of logical "or" ( $\vee$ ) operations.

Table 6. Definition of the STEP PHA buffers at the date of the launch of the mission.

\begin{tabular}{lcc}
\hline \hline PHA buffer \# & Sensor channel & Description \\
\hline $0-2$ & & Single hits \\
3 & & $7 \mathrm{keV}<E<9 \mathrm{keV}$ (Cu X-ray line) \\
4 & IC & $E>70 \mathrm{keV}$ (MIPS) \\
5 & & $E<2 \mathrm{keV}$ (noise) \\
6 & Wrong pixel size \\
7 & & Multiple hits \\
\hline $8-10$ & $\mathrm{MC}$ & Single hits \\
11 & & $7 \mathrm{keV}<E<9 \mathrm{keV}$ (Cu X-ray line) \\
12 & $E>70 \mathrm{keV}$ (MIPS) \\
13 & $E<2 \mathrm{keV}$ (noise) \\
14 & & Wrong pixel size \\
15 & & Multiple hits \\
\hline
\end{tabular}

Notes. It is expected that buffers 6 and 14 will remain empty, unless there is a problem with the configuration or data processing of the sensor.

Table 7. Data products of STEP at time of launch.

\begin{tabular}{lrrrl}
\hline \hline Telemetry channel & Energy range $(\mathrm{keV})$ & \# bins & Cadence (far/close) (s) & Pixels layout $^{(a)}$ \\
\hline Nominal & & & & \\
& $2-80$ & 8 & $60 / 10$ & Individual pixels: $0,1, \ldots 15$ \\
& $2-60$ & 4 & $5 / 1$ & Three Rows of five pixels \\
& $2-60$ & 4 & $5 / 1$ & Five Columns of three pixels \\
& $2-60$ & 4 & $5 / 1$ & Background pixel: 0 \\
& $2-80$ & 48 & $60 / 10$ & Sum of pixels 1-15 \\
& N/A & N/A & $60 / 10$ & Hit multiplicities \\
\hline Low latency & $2-80$ & 24 & & \\
& $2-80$ & 24 & 60 & Sum of pixels 1-15 \\
& $2-60$ & 1 & 60 & Background pixel: 0 \\
& $2-60$ & 1 & 1 & Sum of pixels 1-15 \\
& & & 1 & Background pixel: 0 \\
\hline Burst & $2-80$ & 16 & 1 & Individual pixels: $0,1, \ldots 15$ \\
& $2-80$ & 48 & 1 & Sum of pixels 1-15 \\
\hline
\end{tabular}

Notes. ${ }^{(a)}$ See Fig. 6.

It is possible through telecommanding to alter and expand the operational modes presented here, in order to tailor the operation of the sensor to other scientific or operational requirements.

\section{The Electron Proton Telescope (EPT)}

\subsection{Introduction}

The EPT relies on the magnet/foil technique adapted from STEREO/SEPT (Müller-Mellin et al. 2008) to separate and measure electrons and protons in the energy range shown in Fig. 3. The EPT will cover the gap between low- and high-energy particles measured by STEP and HET, respectively. The energy range and energy resolution as well as the time resolution and pointing directions are optimised to provide crucial constraints on the acceleration and propagation of energetic particles.

The EPT consists of two double-ended telescopes and is integrated together with HET into a unit with a common housing and read-out electronics (Fig. 14). There are two EPT-HET units that provide directional information about the incoming particles: EPT-HET 1 is pointing sunward and anti-sunward along the nominal Parker spiral and is located on the $-y$ deck of the spacecraft, while EPT-HET 2 is pointing northward and southward and is located on the $+y$ deck of the spacecraft (for FoVs see Fig. 4). 


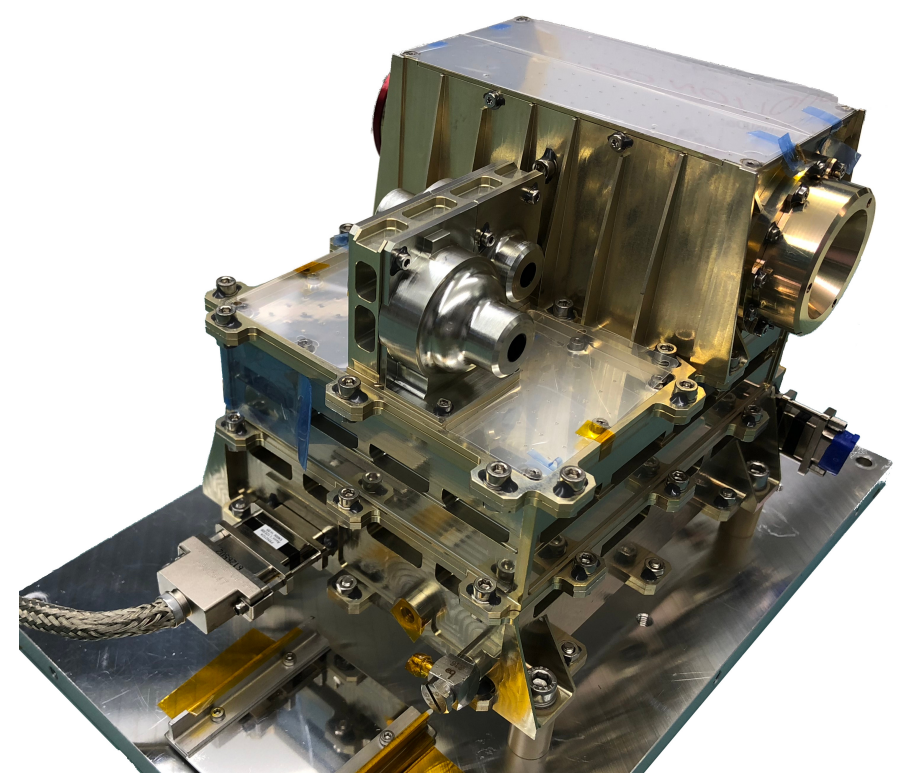

Fig. 14. EPT-HET FS unit. The EPT is on the left and the HET is the big telescope on the right; the lower part contains the electronics and power-supply.

\subsection{Description of the Electron Proton Telescope}

\subsubsection{Operating principles and design}

The schematics of EPT are shown in Fig. 15 and its block diagram is shown in Fig. 16. Each EPT unit has two double-ended telescopes with back-to-back mounted, segmented, silicon-based SSDs. Each SSD has a circular central segment with a diameter of $6 \mathrm{~mm}$ and a ring-shaped outer anti-coincidence segment with a diameter of $12 \mathrm{~mm}$. For the detection of stopping particles the two central segments also serve as anti-coincidence for one another. One of the SSDs is covered with a $5 \mu \mathrm{m}$ polyimide absorption foil and its partner looks through the air gap of a magnet system.

The magnet system consists of two pairs of VACODYM $677 \mathrm{HR}$ neodymium permanent magnets. Each pair leaves an air gap with the necessary magnetic induction $(B=0.4 \mathrm{~T})$ to deflect low-energy electrons. The long-range field is attenuated by placing the two oppositely polarised magnetic dipoles at close distance. Thus, the two air gaps provided by the magnet system simultaneously serve two telescope systems with anti-parallel viewing directions, at minimum weight penalty, while satisfying the stringent requirements on magnetic cleanliness.

The foil leaves the electron spectrum above $30 \mathrm{keV}$ essentially unchanged but stops protons of energy up to $\sim 400 \mathrm{keV}$, that is, the energy of electrons that penetrate the first SSD. The magnet is designed to sweep away electrons below $400 \mathrm{keV}$, but leaves ions unaffected. In the absence of $>400 \mathrm{keV}$ protons/ions, the foil SSD detects only electrons, and the magnet SSD detects only protons/ions.

The predecessor of the EPT, STEREO/SEPT, suffered from significant background noise from galactic cosmic rays coming through the telescope housing without triggering the anticoincidence. To mitigate this on EPT the detectors are much closer together $(0.4 \mathrm{~mm})$ and the cross-talk guard ring between the inner and outer segments is read out together with the outer segment as an anti-coincidence. This reduces the geometric factor for the cosmic-ray background.

The geometric factor calculated analytically according to Sullivan (1971) is listed in Table 8. A detailed response of

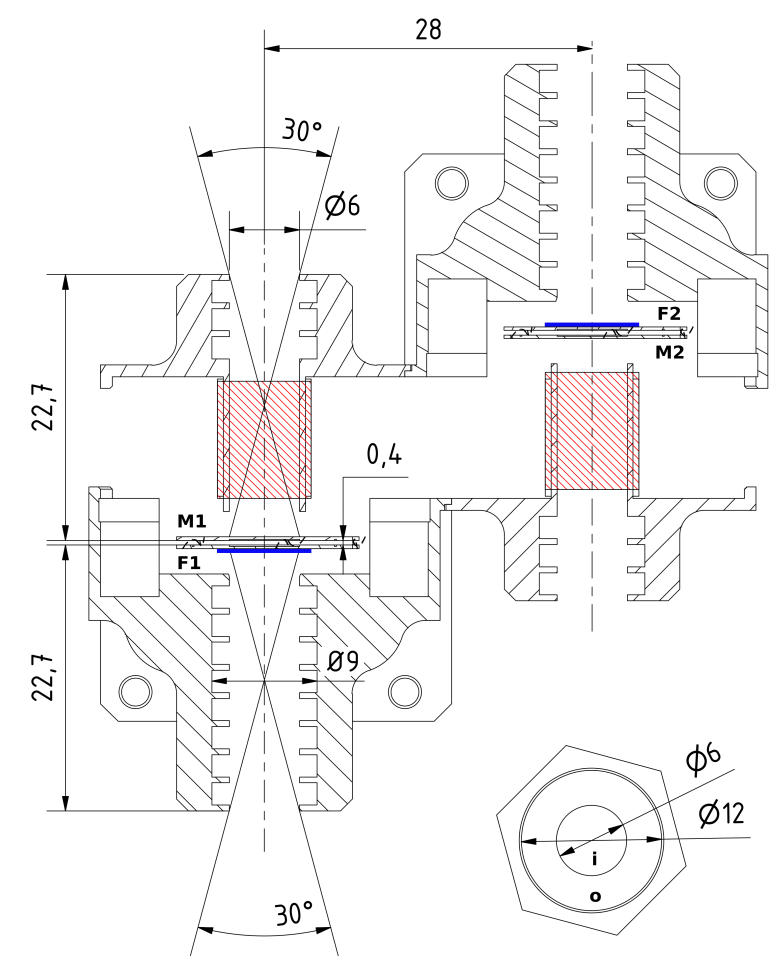

Fig. 15. Horizontal cut of the EPT sensor with distances in mm. Red is the magnetic field perpendicular to the drawing, blue indicates where the $5 \mu \mathrm{m}$ polyimide layer is located. The detectors are named M1, F1, M2, and F2 (for magnet and foil) and their inner and outer segments are denoted by $i$ and o, respectively: r.g. M2i.

the instrument has been simulated using the Geant4 toolkit (Agostinelli et al. 2003) and is presented in Sect. 5.3.2.

\subsubsection{Event analysis and particle identification for the Electron Proton Telescope}

The EPT uses passivated implanted planar silicon (PIPS) detectors from Canberra Semiconductors, each $300 \mu \mathrm{m}$ in thickness. These are coated with a $100 \mathrm{~nm}$ layer of aluminum to shield them from stray light and are operated fully depleted with a reverse bias voltage of $+70 \mathrm{~V}$. Each detector is segmented into an inner segment, a cross-talk guard ring, and an outer segment. The last two are read out together and serve as an anti-coincidence.

The SSDs emit a pulse of charge within a few nanoseconds after a particle hits the detector. Charge-sensitive pre-amplifiers (CSA) convert the charge pulse into a voltage step. The gain is defined by the inverse of the feedback capacitance. The feedback capacitance discharges with a time constant of $100 \mu \mathrm{s}$. Simple single pole shapers with partial pole-zero correction convert the voltage steps into unipolar pulses. The shapers provide additional gain.

The EPT uses only one shaper per detector segment. The shaper outputs are sampled directly by ADC at 1 million samples per second.

The level-1 trigger in the analogue board FPGA applies a linear combination $A=\Sigma a_{i} s_{i}$ of the last 15 samples to provide a digital measure for the pulse height in every microsecond. The coefficients can take values from -3 to +3 . The sum of the coefficients must be zero. A second linear combination $B$ provides a measure for the phase of the pulse with respect to the ADC clock. The coefficients used for EPT (and HET) are $a_{i}=(0,0,0,-3,-3,-3,-3,1,1,2,2,3,2,1,0)$ and 


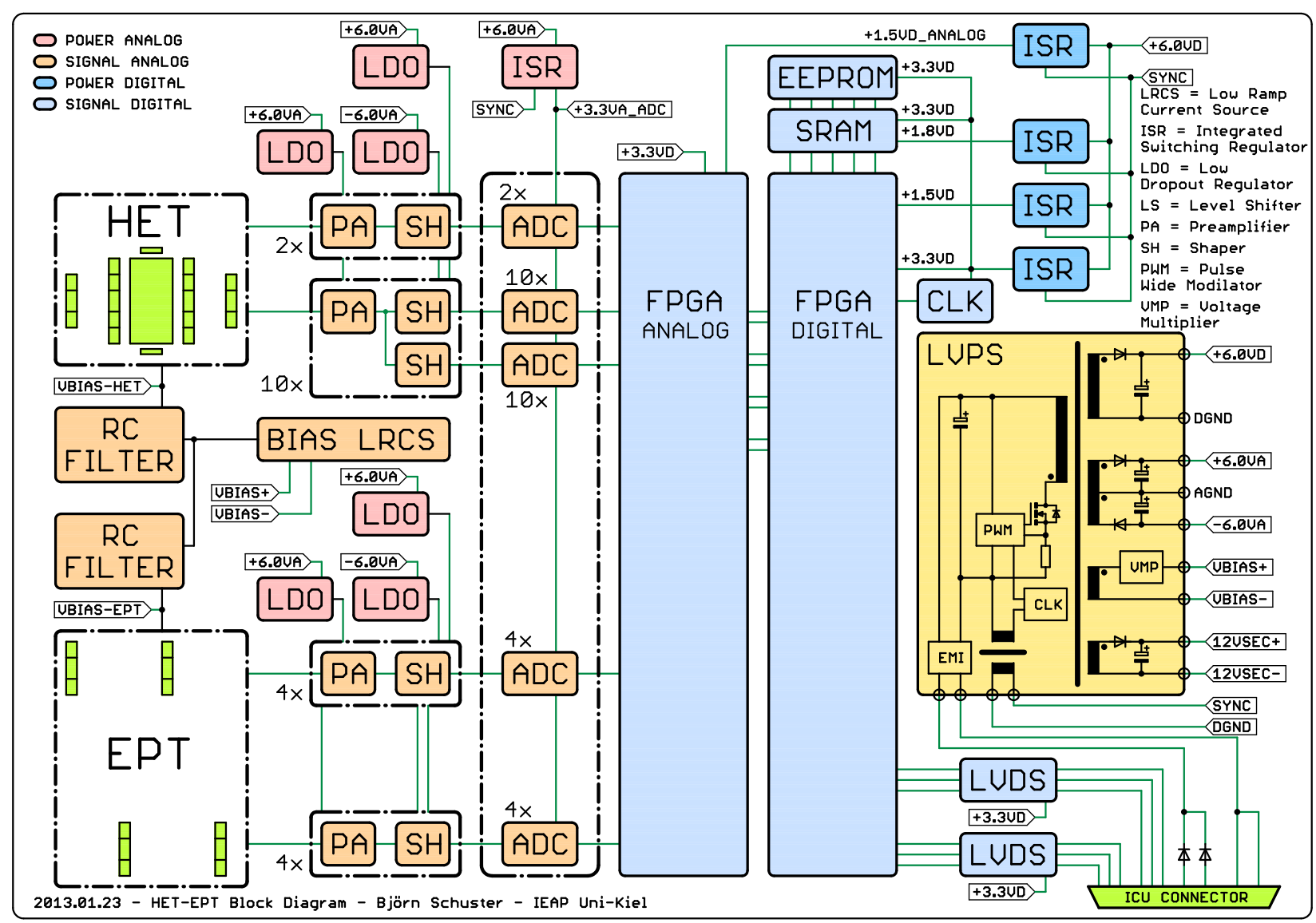

Fig. 16. Block diagram of the Electron Proton Telescope and High-Energy Telescope.

Table 8. Geometric factors of EPT.

\begin{tabular}{lc}
\hline \hline Cases & Geometric factor $\left(\mathrm{mm}^{2} \mathrm{sr}\right)$ \\
\hline Stopping particles & 1.540 \\
Penetrating particles & 1.451 \\
\hline
\end{tabular}

$b_{i}=(0,0,0,0,0,0,0,-2,-3,-1,0,2,2,1,1)$. These coefficients can be changed in flight, but there is only one set for all channels. Any change may invalidate all previous calibration measurements.

The level-1 trigger then compares the reconstructed pulse heights to configurable thresholds and flags the detectors with signal amplitudes above the thresholds.

Additionally, the FPGA can be configured with up to eight different coincidence triggers (level-2 triggers), based on a combination of the flagged detectors. The level-2 triggers have configurable coincidence timing windows. After a peak is detected in one channel, the system waits for a minimum time of $2 \mu \mathrm{s}$ and a maximum time of $4 \mu$ s to see if other channels have also seen a peak and the trigger conditions are fulfilled. Otherwise after $6 \mu \mathrm{s}$ the peak is discarded.

Each level-2 trigger is mapped to one of four distinct level3 trigger classes. These trigger classes control which section of the final level-3 trigger engine will be executed. Table 9 lists the configured level-2 triggers for EPT.

The level-3 trigger engine is common to the EPT/HET and STEP sensors, and is a fully flight-configurable assembly-like instruction set implemented in the digital board FPGA. The
Table 9. EPT Level 2 trigger logic.

\begin{tabular}{ll}
\hline \hline Coincidence condition & Description \\
\hline$(M 1 i \vee F 1 i) \wedge \overline{M 1 o \vee F 1 o}$ & EPT telescope 1 \\
$(M 2 i \vee F 2 i) \wedge \overline{M 2 o \vee F 2 o}$ & EPT telescope 2 \\
\hline
\end{tabular}

Notes. The labels of the coincidences correspond to the labels of the detectors in EPT. Both EPT L2 triggers will activate trigger class 0 of the Level-3 trigger.

purpose of the level-3 trigger is to control the creation of histograms, which are the main data products of a sensor, as well as to control the creation of PHA buffers. For each trigger class, up to 256 instructions can be processed.

The EPT level-3 trigger calibrates all channels to kiloelectronvolt energies and determines the inner segment of each telescope that has measured the maximum deposited energy. It then checks if it is either a valid stopping event (less than $1 \%$ of maximum energy deposited in outer segments and other inner segment) or a valid penetrating event (less than $1 \%$ of maximum energy deposited in outer segments, more than $1 \%$ deposited in other inner segment).

Stopping events are stored in logarithmic histograms with a bin width of 16 bins per octave. Penetrating events are stored in a two-dimensional histogram of total deposited energy versus ratio of energy deposited in the two inner segments (see Fig. 17). This allows us to separate particles by direction, species, and energy, though the energy resolution is not as good as for stopping particles. 


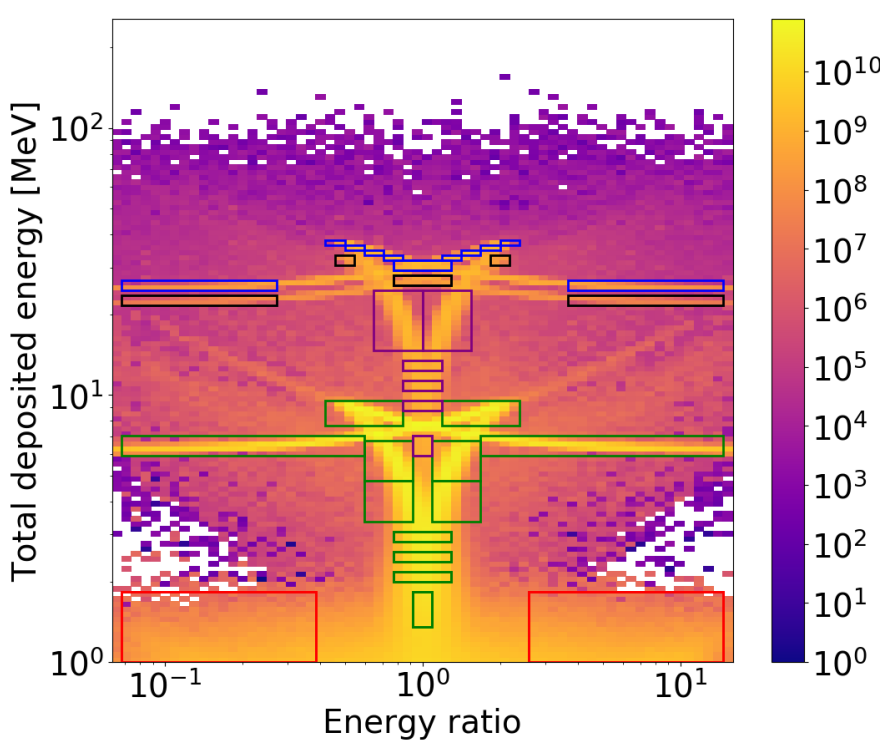

Fig. 17. EPT level-3 penetrating histogram of Geant4-simulated data Data products are sums of the overlaid boxes (red: $\mathrm{e}^{-}$, green: $\mathrm{H}$, black: ${ }^{3} \mathrm{He}$, blue: ${ }^{4} \mathrm{He}$, purple: combined $\mathrm{He}$ ). The diagonal blue boxes are connected.

Each event is also assigned to one out of 16 PHA classes (for EPT and HET together) and is sent to a corresponding $1 \mathrm{kB}$ buffer, which will store the full PHA record of events until it is full and also has a counter of all events sent to the buffer.

Since EPT and HET share a common electronics box, the FPGA board as well as the Low-Voltage Power Supply (LVPS) are commonly used by EPT and HET sensor heads. The electronic block diagram of EPT and HET units is shown in Fig. 16.

\subsubsection{Data products of the Electron Proton Telescope}

The EPT produces several types of data products:

- Nominal data: This consists of compressed histograms of stopping and penetrating particles and various cadences and energy resolutions; see Table 10 for the configuration at launch.

- Low-latency data: A subset of low-resolution histogram data is sent to Earth once a day; see Table 10.

- Burst-mode data: The EPT constantly produces extra-highresolution data which is sent to the ICU, a subset of which can then be selected for transmission to Earth. The products are listed in Table 10.

- PHA data: The EPT uses the three PHA buffers 0,1 , and 2 for invalid, penetrating, and stopping events, respectively. These $1 \mathrm{kB}$ buffers will be sent at least once an hour and serve primarily for long-term calibration and instrument health monitoring, but can also be made available for science data.

- Housekeeping data: This includes temperatures and voltages, single channel trigger counters, error counters, and more.

\subsubsection{Operating modes of the Electron Proton Telescope}

The EPT sensor can be operated in the following modes:

- Off: Off.

- Housekeeping: Only generation of housekeeping packets is enabled. Data generation is disabled.

- Operational - Low flux: Nominal operational mode with default threshold configurations and generation of nominal data products, housekeeping and PHA data.
- Operational - High flux: Configuration for high particle flux. Detector thresholds for the inner segments are increased from $25 \mathrm{keV}$ to $45 \mathrm{keV}$.

\subsection{Performance of the Electron Proton Telescope}

\subsubsection{Energy resolution}

The eight detectors making up the four stacks of two EPT FM instruments were selected from a total of 36 SSD devices supplied by Canberra Semiconductor. Each individual detector was tested with conversion electrons from a radioactive ${ }^{207} \mathrm{Bi}$ source using standard lab electronics at ambient conditions. All detectors show an energy resolution of less than $7 \mathrm{keV}$ FWHM. Figure 18 shows the result for the central segment of one EPT foil detector. In this plot one can see all the prominent electron, X-ray, Auger electron peaks and the minimally ionizing peak at $95 \mathrm{keV}$. The $56 \mathrm{keV}$ Auger peak appeared at lower energy because of some energy loss in the $5 \mu \mathrm{m}$ polyimide foil.

\subsubsection{Efficiency}

The EPT sensor was modelled with a Geant4-based Monte Carlo simulation to model the detection efficiencies for protons, electrons, and helium over a wide energy range for both the magnet side and the foil side. It is especially important to know the false-positive detection efficiency of electrons on the magnet side and protons on the foil side so that we can later correct for it on the ground. Figure 19 shows the simulated geometric factor for stopping electrons and protons in both magnet side and foil side. Figure 20 shows the response matrix of incoming versus deposited energy for these particle populations.

\subsection{Calibration of the Electron Proton Telescope}

The EPT level-3 trigger needs to convert the values read out by the ADC to kiloelectronvolts on-board. For this purpose the unit configuration contains a set of calibration factors for different temperature points. The calibration factor results from the individual SSD detector efficiency as well as the electronics gain depending on the value of discrete resistors and capacitors, the resulting time constants and pulse shapes.

The EPT FMs were calibrated with a ${ }^{207} \mathrm{Bi}$ source in a thermal vacuum chamber. The calibration factors for the EPT detectors at $0^{\circ} \mathrm{C}$ are listed in Table 11 and we derived the following best-fit temperature calibrations for EPT 1 and EPT 2 (with T in ${ }^{\circ} \mathrm{C}$ and $\mathrm{C}_{0}$ taken from Table 11).

EPT1 $: C(T)=C_{0} /\left(1-9.756 \times 10^{-5} \cdot T+7.456 \times 10^{-6} \cdot T^{2}\right)$

EPT2 $: C(T)=C_{0} /\left(1+1.453 \times 10^{-4} \cdot T+5.111 \times 10^{-6} \cdot T^{2}\right)$

Figure 21 shows the performance of the fully integrated EPT 1 unit with a ${ }^{207} \mathrm{Bi}$ source in front of the foil-side detector F1. Energy loss of the electrons in air is considered for this calibration. In this figure we see prominent conversion electron peaks of the ${ }^{207} \mathrm{Bi}$ source in detector F1i, while M1i sees only the high-energy electrons of the source (shifted leftward due to energy loss in F1i). The other two detectors (F2i and M2i) only see X-rays and Compton edges of ${ }^{207} \mathrm{Bi} \gamma$-rays and no conversion electrons.

In addition the (flight-like) EPT PQM model was calibrated with mono-energetic proton and alpha beams using the accelerator facility at Physikalisch-Technische Bundesanstalt (PTB) in Braunschweig, Germany. Figure 22 shows protons of different energies hitting the magnet-side detector. Meanwhile Fig. 23 
Table 10. EPT data products for each FoV.

\begin{tabular}{|c|c|c|c|c|c|c|}
\hline \multirow[t]{2}{*}{ Species } & \multicolumn{3}{|c|}{ Nominal } & \multicolumn{2}{|c|}{ Low-latency } & \multirow[t]{2}{*}{ Burst } \\
\hline & Stopping & Penetrating & High cadence & Stopping & High cadence & \\
\hline \multicolumn{7}{|l|}{$\mathrm{e}^{-}$} \\
\hline Energy range (MeV) & $0.025-0.475$ & $1-5$ & $0.025-0.475$ & $0.025-0.4$ & $0.05-0.1$ & $0.025-0.475$ \\
\hline Cadence (s) & 60 & 5 & 1 & 30 & 5 & 1 \\
\hline \# of bins & 34 & 2 & 17 & 8 & 1 & 34 \\
\hline \multicolumn{7}{|l|}{$\mathrm{H}$} \\
\hline Energy range (MeV) & $0.025-6.4$ & $6-50$ & $0.025-6.4$ & $0.025-6.4$ & $0.05-0.2$ & $0.025-6.4$ \\
\hline Cadence (s) & 5 & 5 & 1 & 30 & 5 & 1 \\
\hline \# of bins & 64 & 8 & 12 & 16 & 2 & 64 \\
\hline \multicolumn{7}{|l|}{${ }^{3} \mathrm{He},{ }^{4} \mathrm{He}$} \\
\hline Energy range $\left(\mathrm{MeV} \mathrm{n}^{-1}\right)$ & - & $6-10$ & - & - & - & $6-10$ \\
\hline Cadence $(s)$ & - & 30 & - & - & - & 5 \\
\hline \# of bins & - & $2 \times 3$ & - & - & - & $2 \times 3$ \\
\hline \multicolumn{7}{|l|}{$\mathrm{He}$} \\
\hline Energy range $\left(\mathrm{MeV} \mathrm{n}^{-1}\right)$ & $1.6-6.4$ & $10-50$ & - & $1.6-6.4$ & - & $1.6-6.4$ \\
\hline Cadence $(\mathrm{s})$ & 5 & 30 & - & 30 & - & 1 \\
\hline \# of bins & 8 & 5 & - & 2 & - & 8 \\
\hline
\end{tabular}

Notes. Nominal data product cadences are applicable close to the sun and will be increased at greater distances. Low-latency data are constant and will be downloaded once a day. These are used for mission planning. Burst data products will be provided in addition to the nominal data when burst mode is activated.

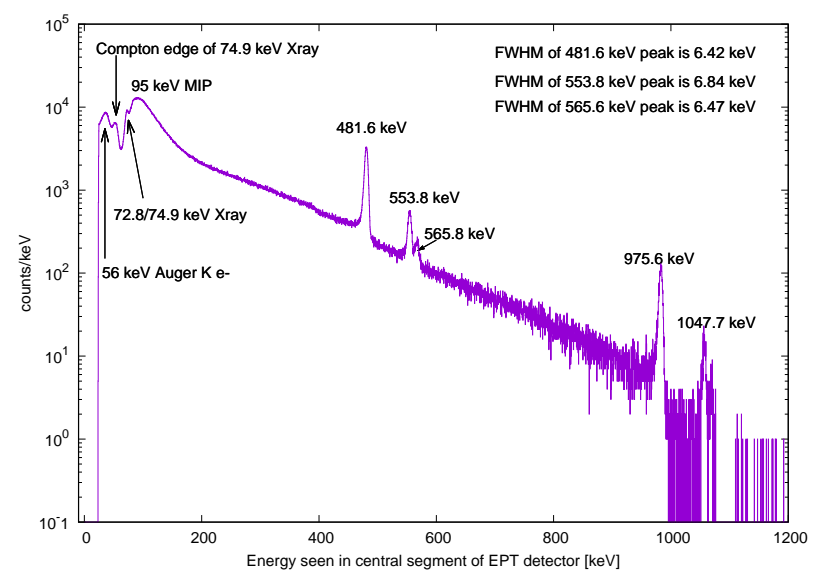

Fig. 18. Energy resolution of an EPT foil-side detector with a ${ }^{207} \mathrm{Bi}$ source measured using laboratory equipment prior to assembly.

shows protons on the foil side with protons below $400 \mathrm{keV}$ not able to penetrate the polyimide layer and protons with $424 \mathrm{keV}$ depositing less than $25 \mathrm{keV}$ in the detector.

\section{The Suprathermal Ion Spectrograph (SIS)}

\subsection{Introduction}

The Suprathermal Ion Spectrograph will provide observations of He-Fe between energies just above the solar wind to multiple megaelectronvolt/nucleon energies, with high energies covered by other EPD sensors. The SIS is an advanced instrument based on the high-resolution Ultra-Low Energy Isotope Spectrometer (ULEIS; Mason et al. 1998) instrument on the Advanced Composition Explorer (ACE). Table 12 lists SIS characteristics and a resource summary. The SIS is composed of two telescopes, SIS-A and SIS-B, pointing in different directions and sharing

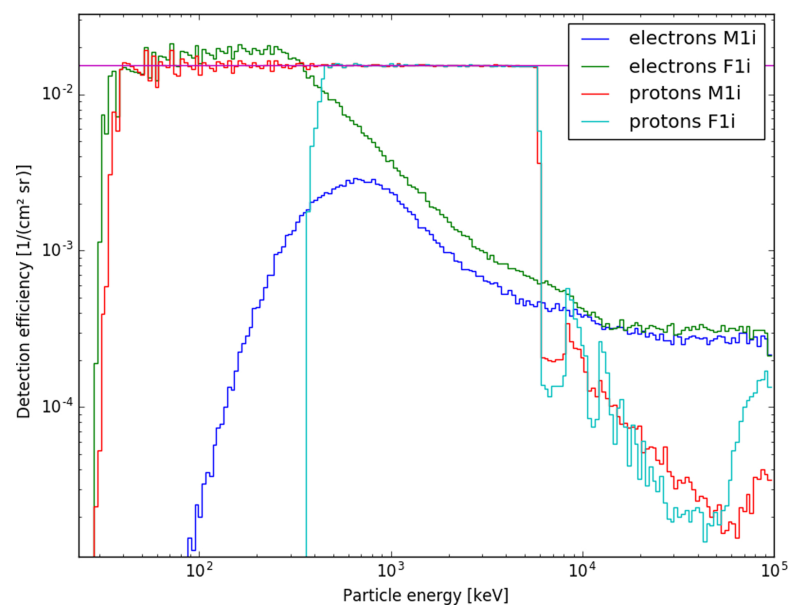

Fig. 19. Geometric factors from simulation of electrons and protons in EPT magnet and foil side detectors in logarithmic binning. The analytical geometric factor is shown in purple.



Fig. 20. Electron Proton Telescope response matrix from Geant4 simulations. 
Table 11. EPT sensor channel name, number, and calibration factor $C_{0}$ at $T=0^{\circ} \mathrm{C}$.

\begin{tabular}{ccccc}
\hline \hline Unit & $\begin{array}{c}\text { Detector } \\
\text { segment }\end{array}$ & $\begin{array}{c}\text { Channel } \\
\text { number }\end{array}$ & $\begin{array}{c}\text { Calibration } \\
C_{0} / \mathrm{keV} / \mathrm{A}\end{array}$ & View \\
\hline EPT1 & $M 2 i$ & 22 & 1.009 & Magnet sun \\
EPT1 & $M 2 o$ & 23 & 1.003 & Magnet sun \\
EPT1 & $F 2 i$ & 24 & 0.990 & Foil anti-sun \\
EPT1 & $F 2 o$ & 25 & 1.004 & Foil anti-sun \\
EPT1 & $F 1 i$ & 26 & 0.992 & Foil sun \\
EPT1 & $F 1 o$ & 27 & 1.013 & Foil sun \\
EPT1 & $M 1 i$ & 28 & 1.005 & Magnet anti-sun \\
EPT1 & $M 1 o$ & 29 & 0.988 & Magnet anti-sun \\
\hline EPT2 & $M 2 i$ & 22 & 0.984 & Magnet south \\
EPT2 & $M 2 o$ & 23 & 0.997 & Magnet south \\
EPT2 & $F 2 i$ & 24 & 0.978 & Foil north \\
EPT2 & $F 2 o$ & 25 & 0.998 & Foil north \\
EPT2 & $F 1 i$ & 26 & 1.006 & Foil south \\
EPT2 & $F 1 o$ & 27 & 0.997 & Foil south \\
EPT2 & $M 1 i$ & 28 & 0.990 & Magnet north \\
EPT2 & $M 1 o$ & 29 & 1.037 & Magnet north \\
\hline
\end{tabular}



Fig. 21. Calibration of EPT FM1 at room temperature with a ${ }^{207} \mathrm{Bi}$ source.

the same electronics box (see Fig. 24). The unit is mounted on the $-y$-deck of the spacecraft.

\subsection{Description}

\subsubsection{Operating principles of the Suprathermal Ion Spectrograph}

Figure 25 shows a cross section of one of the two identical SIS telescopes, which identify particles by time-of-flight mass spectrometry. The instrument simultaneously measures the time of flight (TOF), $\tau$, and residual energy $E$ of ions that enter the $22^{\circ}$ full angle FoV and stop in the silicon solid-state detector at the back. A typical ion path is shown: the ion passes through the entrance, Start-1, Start-2, and Stop foils, emitting secondary electrons at each surface. The electrons from the Start-1, Start2 , and Stop foils are accelerated by $\sim 1 \mathrm{kV}$ and directed via isochronous trajectories onto microchannel plates (MCPs) that provide fast Start-1, Start-2, and Stop signals. The timing signals are used to measure the ion TOF between foil combinations. The three times of flight measured are: Start-1 to Stop (=TOF-1);

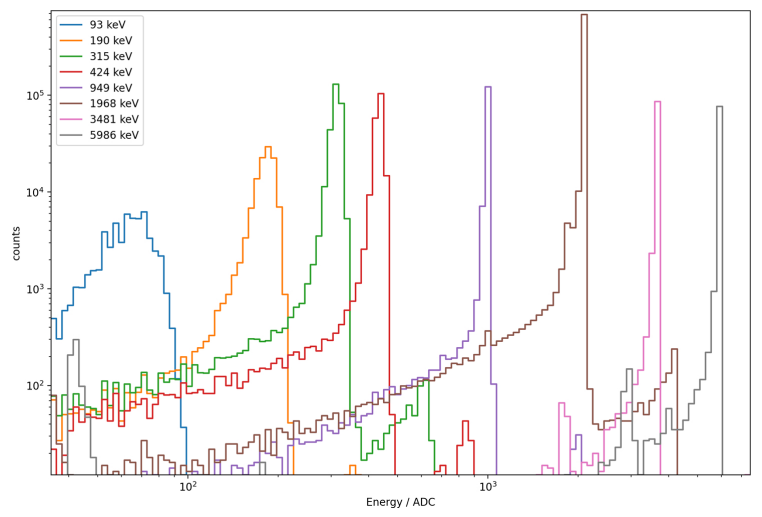

Fig. 22. Calibration of EPT PQM at PTB with protons hitting the magnet side.

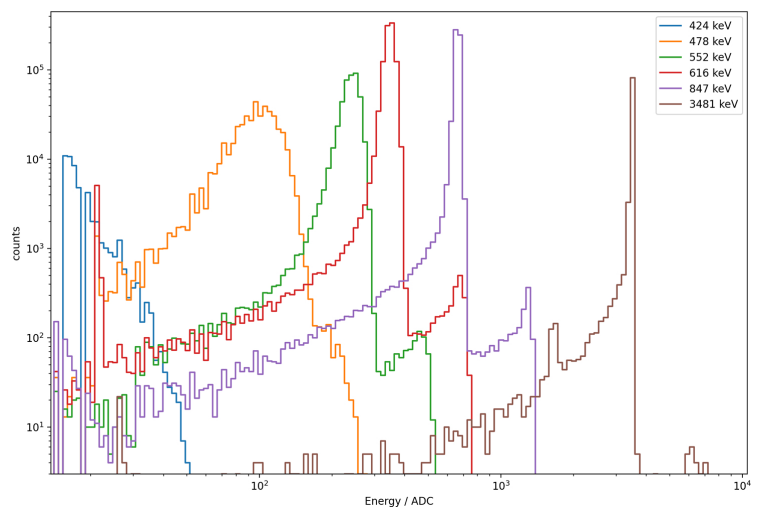

Fig. 23. Calibration of EPT PQM at PTB with protons hitting the foil side.

Table 12. SIS resource summary.

\begin{tabular}{|c|c|}
\hline Item & Description \\
\hline Mass $(\mathrm{kg})^{(a)}$ & 6.816 \\
\hline Power $(\mathrm{W})^{(b)}$ & 3.800 \\
\hline Survival heater power (W) & 4.07 \\
\hline Door power $(\mathrm{W})$ & 10.035 each \\
\hline Bit rate $\left(\text { bits s }{ }^{-1}\right)^{(a)}$ & 590 \\
\hline
\end{tabular}

Notes. ${ }^{(a)}$ Nominal allocation. ${ }^{(b)}$ All modes.

Start-2 to Stop (=TOF-2); and Start-1 to Start-2 (=TOF-3). For an ion with measured energy $E=\frac{1}{2} m v^{2}$, and velocity $v=L / \tau$, the mass can be determined using:

$m=2 E\left(\frac{\tau}{L}\right)^{2}$,

where $L$ is the ion flight path. The measured speed in the telescope, $v$, gives the energy per nucleon: $E / m=v^{2} / 2$. After correcting for losses in the foils, the incident energy per nucleon of the ion is obtained.

\subsubsection{Description of the Suprathermal Ion Spectrograph telescope}

Sunshade and telescope door. Each of the two SIS telescopes has a sunshade in front to limit the access of both particles and stray light to the front foil in order to reduce background. The boresights of the sunward (SIS-A) and anti-sunward 


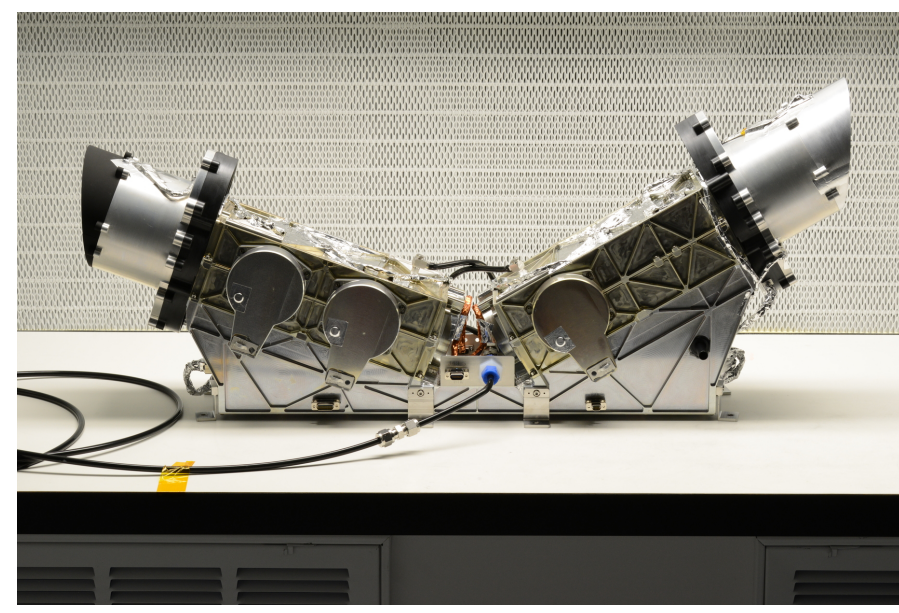

Fig. 24. Side view of the SIS Flight Model.

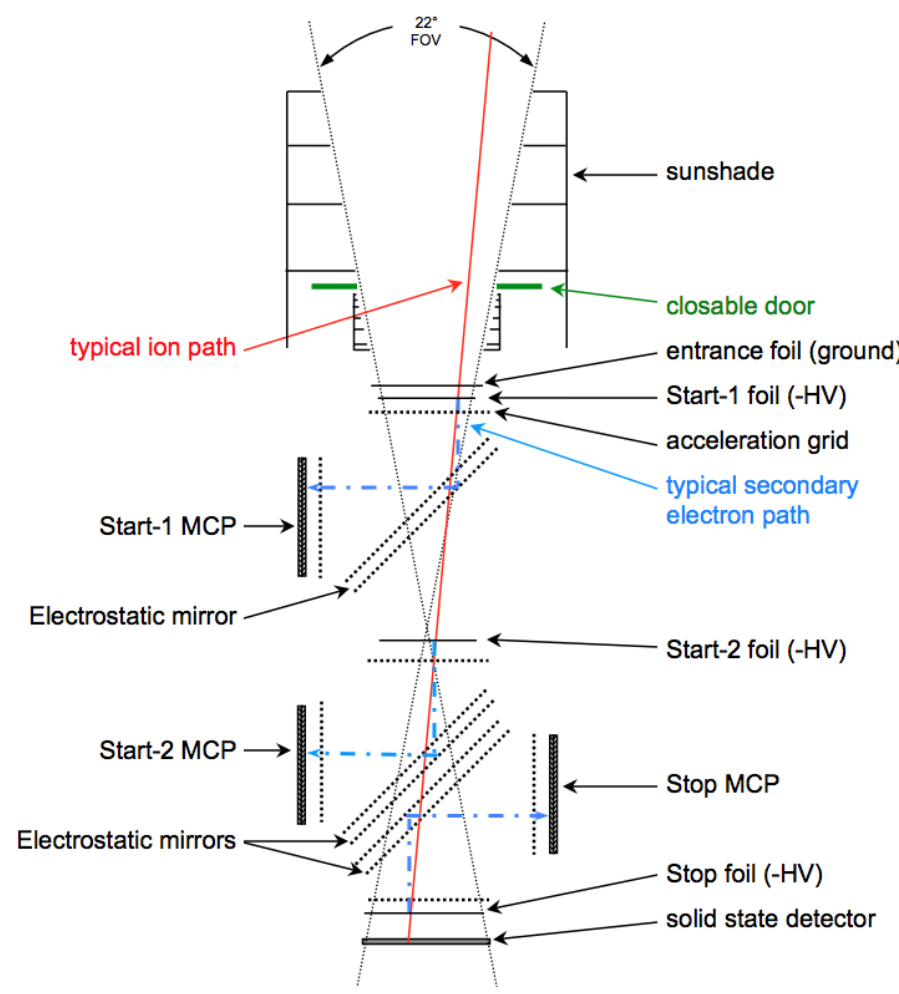

Fig. 25. Schematic cross section of SIS telescope. The two telescopes point $30^{\circ}$ (SIS-A; sunward) and $160^{\circ}$ (SIS-B; anti-sunward) to the west of the spacecraft-Sun line.

(SIS-B) telescopes are pointed $30^{\circ}$ and $160^{\circ}$ to the west of the spacecraft-Sun line, respectively. With this pointing, the sunward telescope $22^{\circ}$ full cone angle will intersect nominal interplanetary magnetic field (IMF) directions for most combinations of solar wind speed and radial distance from the Sun. In addition, each telescope has an independently controlled stepper motor driven iris door whose primary purpose in flight is to prevent instrument saturation by closing down the telescope aperture during intense events, similar to ACE/ULEIS. The doors move between four fixed settings ( $100 \%$ open, $25 \%, 5 \%$, and $1 \%$ ) using an onboard algorithm which improves the one used on the ACE/ULEIS doors. The sunward-facing telescope door is also used to protect the front foils in case of a spacecraft off-pointing condition. Figure 26 shows the SIS telescope with and without
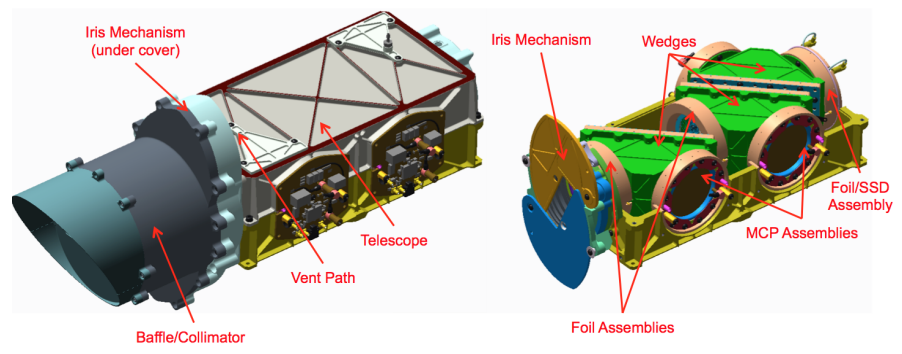

Fig. 26. Single SIS telescope with (left) and without (right) housing. All the major components of the telescope are identified.

Table 13. Geometric factors of the SIS telescope.

\begin{tabular}{lcc}
\hline \hline Element & SIS-A & SIS-B \\
\hline Entrance aperture diameter & $4.0 \mathrm{~cm}$ \\
Stop foil aperture diameter & $4.0 \mathrm{~cm}$ \\
Separation of Entrance aperture and Stop foil & $20.775 \mathrm{~cm}$ \\
Geometric factor before losses & $0.360 \mathrm{~cm}^{2} \mathrm{sr}$ \\
Acceptance cone full angle & \multicolumn{2}{c}{$22.0^{\circ}$} \\
Transparency factor & 0.6121 \\
Loss factor due to aperture reduction plate & $26.94 \%$ & $0.28 \%$ \\
Net geometric factor & $0.161 \mathrm{~cm}^{2} \mathrm{sr}$ & $0.220 \mathrm{~cm}^{2} \mathrm{sr}$ \\
\hline
\end{tabular}

Notes. All but the last two properties are identical for both apertures.

the housing cover showing major components such as the baffle and iris door.

Geometric factor/harp and grid transmission. The SIS geometric factor is defined by the entrance aperture $(40.0 \mathrm{~mm}$ diameter), the stop foil aperture (40.0 $\mathrm{mm}$ diameter), and their separation $(206 \mathrm{~mm})$. The geometric factor before losses is therefore $0.358 \mathrm{~cm}^{2} \mathrm{sr}$, and the full cone angle is $22^{\circ}$. The geometric factor is reduced by the transparency of grids $(\sim 0.61)$ that support the foils and constitute the electrostatic mirrors. Furthermore, an aperture reduction plate was installed in both telescope sunshades to prevent stray light from entering the telescopes and generating background counts from the front foil. These aperture plates were specifically designed to mitigate known spacecraft structures around the telescope, and reduced the instrument geometric factor further. The final geometric factor of the instrument is listed in Table 13.

Telescope foils. Each SIS telescope has four foils: the entrance and Start-1 foil are Ni on polyimide mounted on a supporting mesh. Ni was chosen for its low UV transmission. The Start-2 and Stop foils are Al supported on polyimide and a supporting mesh. The metallic surfaces are the secondary electron emitting surfaces, and the foils are mounted such as the metal surface faces the accelerating harp in each case. Both $\mathrm{Ni}$ and Al have low efficiency for secondary electron emission, which greatly reduces proton triggering, thereby significantly lowering the count rates inside the instrument (protons are measured by other instruments in the Solar Orbiter EPD suite). The SIS foil details and nominal $\mathrm{Si}$ detector thicknesses are shown in Table 14.

MCPs/SSDs. Micro-channel plates are used to measure the timing of the secondary electrons. Each MCP assembly consists of a chevron pair of plates of $40 \mathrm{~mm}$ diameter active area. The Start-1, Start-2, and Stop MCP assemblies are all identical. The silicon ion-implant solid-state detector (SSD) at the end of the SIS telescope consists of a single circular pixel that is used to cover the overall $44 \mathrm{~mm}$ diameter detector plane while 
Table 14. Material in SIS FM telescopes ion path.

\begin{tabular}{|c|c|c|c|}
\hline Element & Material & SIS-A thickness $(\AA)$ & SIS-B thickness $(\AA)$ \\
\hline \multicolumn{4}{|l|}{ Entrance foil: } \\
\hline $\mathrm{Ni}$ on substrate & $\mathrm{Ni}$ & 1308 & 1288 \\
\hline Substrate on mesh & Polyimide & 1550 & 1535 \\
\hline \multicolumn{4}{|l|}{ Start1 foil: } \\
\hline Substrate on mesh & Polyimide & 1513 & 1464 \\
\hline Ni on Substrate & $\mathrm{Ni}$ & 1308 & 1308 \\
\hline \multicolumn{4}{|l|}{ Start2 foil: } \\
\hline Substrate on mesh & Polyimide & 1507 & 1423 \\
\hline Al on Substrate & $\mathrm{Al}$ & 316 & 316 \\
\hline \multicolumn{4}{|l|}{ Stop foil: } \\
\hline Al on Substrate & $\mathrm{Al}$ & 312 & 316 \\
\hline Substrate on mesh & Polyimide & 1557 & 1423 \\
\hline $\begin{array}{l}\text { Total absorber in fror } \\
\text { layer: }\end{array}$ & nt of active & 9371 & 9073 \\
\hline
\end{tabular}

Table 15. SIS detector specifications and performance.

\begin{tabular}{lc}
\hline \hline SSD & \\
Type & Silicon ion implant \\
Supplier & Micron Semiconductor Ltd \\
Active area & $15.2 \mathrm{~cm}^{2}(44.0 \mathrm{~mm}$ dia $)$ \\
Thickness & $700 \pm 50 \mu \mathrm{m}$ \\
Mounting substrate & Alumina \\
Active surface metallization & 0 \\
Junction dead layer & $0.05 \mu \mathrm{m}$ \\
Capacitance & $\sim 225 \mathrm{pF}$ \\
Depletion voltage & $\sim 100 \mathrm{~V}(\mathrm{Not}$ to exceed 200V) \\
Leakage current & $3 \mathrm{~mA} \mathrm{max} \mathrm{at}+55 \mathrm{C}$ \\
60nA typical at $+20 \mathrm{C}$ & $35 \mathrm{keV}$ \\
Alpha width FWHM & $\mathrm{Chevron}$ \\
\hline MCP & PHOTONICS USA, Inc. \\
Type & $50.04(40.00) \mathrm{mm}$ \\
Supplier & $1.50 \pm 0.03 \mathrm{~mm}$ \\
Outside (Active) Diameter & $\mathrm{Nichrome}(80 / 20)$ \\
Thickness & $25 \mu \mathrm{m}$ \\
Electrode material & $12 \pm 1^{\circ}$ \\
Pore size & $1 \times 10^{7} \mathrm{minimum}$ \\
Bias Angle & $5-20 \mu \mathrm{A}$ \\
Gain & $120-480 \mathrm{M} \Omega$ \\
Bias Current Range & \\
Resistance & \\
\hline
\end{tabular}

simultaneously meeting the low noise and dynamic range requirements of the system. The detector is mounted on an alumina substrate, and signals are routed to both high-gain and lowgain amplifiers to cover the large dynamic energy range. Table 15 shows the detector specifications and performance.

\subsubsection{Event analysis and particle identification}

Event analysis. Figure 27 is a block diagram of SIS showing the primary measurements and data flow. Grey areas around each telescope indicate the location of some electronics in the telescopes, while the rest of the electronics are located on two boards in the electronics housing. Each telescope produces identical signal types: three timing signals for each ion (Start-1, Start2, and Stop) are sent to the electronics where they go through constant fraction discriminators (CFDs) which then trigger time-of-flight ASICs that produce three flight time signals: Start1 to Stop; Start-2 to Stop, and Start-1 to Start-2. The three timing paths provide consistency checks for reducing background, and also permit the telescope to return science data with the loss of any anode. In addition to timing signals, one of the MCP stack signals is selected for pulse-width (PW) analysis to assist in rejection of background and allow for monitoring of MCP gain in flight. The MCP PW is a crude measure of pulse height. An ion is considered for analysis if it produces a valid stop (VS): a start/stop pair from one of the three possible flight times (selected by ground command) in a given time window. Each valid event produces 11-bit TOF and PW measurements.

Amplifiers for the SSDs are mounted in the back of the detector to minimise noise. Each detector has one charge amplifier operating continuously. The amplified signal is split into two parts, one with high gain to cover the lower energy range and one with low gain to cover the higher energy range. The shaped output is sent to the event board where it is digitised.

Digital processing. Digitized TOF and SSD values are sent to the event logic and processing section. The processor analyses each event, selecting some PHA events for telemetry, and counting all events according to their mass and energy/nucleon as determined by the PHA data and look-up tables. The data are formatted into telemetry packets and sent to the EPD ICU. The processor also receives commands from the ICU and configures the electronics accordingly.

\subsubsection{Data products of the Suprathermal Ion Spectrograph}

The SIS sensor has five types of output data:

- Hardware rates: Singles and coincidence count rates used for instrument monitoring. Definitions of all the SIS hardware rates are shown in Table 16.

- PHA data: Individual PHA events. The definition of the PHA word is shown in Table 17. The PHA events are the main data product to analyse trace elements (e.g. ${ }^{3} \mathrm{He}$ ), elements where TOF consistency checks and background suppression are critical (e.g. ultra-heavy nuclei), and provide a means to verify the matrix rate box locations.

- Matrix rates: Count rates based on lookup tables for 12 selected species $\left(\mathrm{H},{ }^{3} \mathrm{He},{ }^{4} \mathrm{He}, \mathrm{C}, \mathrm{N}, \mathrm{O}, \mathrm{Ne}, \mathrm{Mg}, \mathrm{Si}, \mathrm{S}, \mathrm{Ca}\right.$ and $\mathrm{Fe}$ ) over 21 energy intervals accumulated in the FPGA processor for $3 \mathrm{~s}, 30 \mathrm{~s}$, and $1800 \mathrm{~s}$ cadence. The energy bins are listed in Table 18.

- He Histogram: Histogram of $\sim 0.5-2.0 \mathrm{MeV} \mathrm{n}^{-1}$ mass range 2.0-6.0 AMU for measuring small amounts of ${ }^{3} \mathrm{He}$ next to the large ${ }^{4} \mathrm{He}$ peak in intense events.

- Housekeeping data: For monitoring of instrument voltage, temperature, table checksums, command status. SIS nominally generates the HK status packet every 1800 s, but is commandable. Each HK packet includes analogue, digital, and software status data.

\subsubsection{Operating modes of the Suprathermal Ion Spectrograph}

The SIS operating modes are defined to accommodate the rest of the instrument suite in EPD. They are:

- Off: Off.

- Standby: Low voltage is on but high voltage has not been ramped up (MCP and SSD bias). 


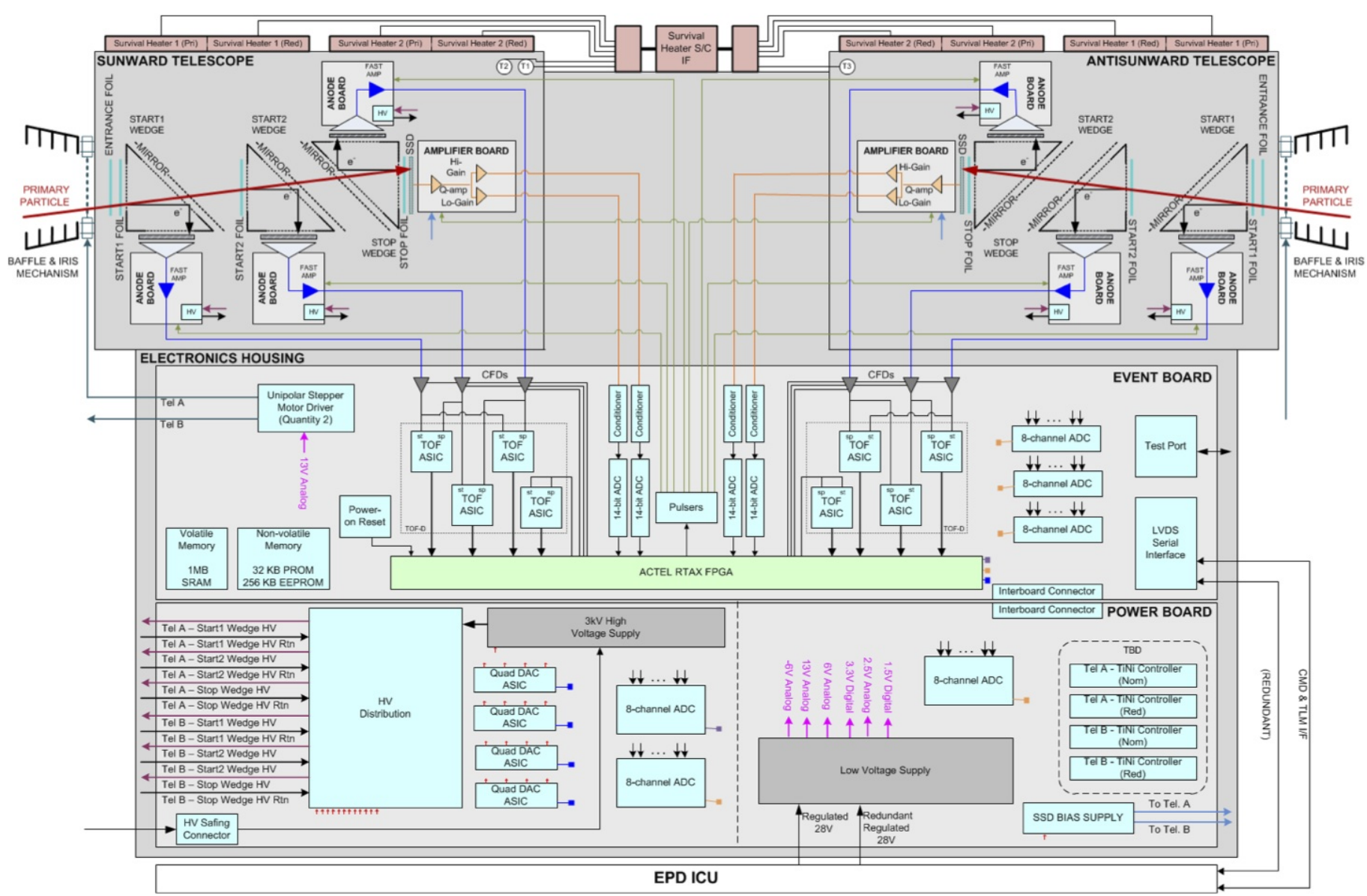

Fig. 27. Block diagram of the Suprathermal Ion Spectrograph.

Table 16. Suprathermal Ion Spectrograph hardware rates.

\begin{tabular}{lcl}
\hline \hline$\#$ & Name & Description \\
\hline 1 & Start 1 CFD & Counts above Start 1 anode threshold \\
2 & Start 2 CFD & Counts above Start 2 anode threshold \\
3 & Stop CFD & Counts above Stop anode threshold \\
4 & Start-1 to Stop TOF VE & Valid event \\
5 & Start-2 to Stop TOF VE & Valid event \\
6 & Start-1 to Start-2 TOF VE & Valid event \\
7 & Energy Low & Counts above SSD low gain threshold \\
8 & Energy High & Counts above SSD high gain threshold \\
9 & Valid Coincidence & TOF - E coincidence \\
10 & FIFO writes & \\
\hline
\end{tabular}

- Operational: Normal mode operation: default case. Enable all matrix, helium histogram data with also limited HK and PHA data.

- Burst (high cadence): Burst mode for SIS is a $3 \mathrm{~s}$ cadence compared to the normal $30 \mathrm{~s}$ cadence. Only Basic Rates and Matrix Rates packets are produced at burst mode cadence. These packets are continuously generated and sent to the EPD ICU. A burst-mode flag is also sent to the ICU to indicate high-intensity periods in SIS data that are candidates for telemetering highcadence packets.

- Diagnostic: Used for instrument troubleshooting and reconfiguration. In this mode, only raw PHA packets are telemetered.
Table 17. SIS telemetered PHA event contents.

\begin{tabular}{lcc}
\hline \hline Item no. & Contents & Bits \\
\hline 1 & TOF-1 $^{(a)}$ & 11 \\
2 & TOF-2 $^{(b)}$ & 11 \\
3 & TOF-3 $^{(c)}$ & 11 \\
4 & SSD energy & 12 \\
5 & SSD ramp $(0=\text { high gain })^{(d)}$ & 1 \\
6 & Test pulser on/off (1/0) $^{(1 / 0)}$ & 1 \\
7 & Priority $^{(e)}$ & 2 \\
8 & MCP pulse size $^{(e)}$ & 7 \\
\hline
\end{tabular}

Notes. There is no telescope ID bit since PHA events go into separate packets for each telescope. ${ }^{(a)}$ Start-1 Stop. ${ }^{(b)}$ Start-2 Stop. ${ }^{(c)}$ Start-1 Start-2. ${ }^{(d)}$ SSD ramp (gain): $0=$ high; $1=$ low $(E \gtrsim 45 \mathrm{MeV}) .{ }^{(e)} \mathrm{MCP}$ stack whose pulse width is measured is selectable by ground command.

\subsection{Performance of the Suprathermal lon Spectrograph}

\subsubsection{Mass resolution}

Equation (1) shows how mass is calculated from the measured solid state detector signal, $E$, time of flight, $\tau$, and particle path length, $L$. The uncertainty in the mass measurement is then given by

$\left(\frac{\sigma_{\mathrm{m}}}{m}\right)^{2}=\left(\frac{\sigma_{\mathrm{E}}}{E}\right)^{2}+\left(2 \frac{\sigma_{\tau}}{\tau}\right)^{2}+\left(\frac{\sigma_{\mathrm{L}}}{L}\right)^{2}$. 
Table 18. Suprathermal Ion Spectrograph energy boxes for both telescopes. Incident energy in $\mathrm{MeV} \mathrm{n}^{-1}$.

\begin{tabular}{lccc}
\hline \hline Energy box \# & $E_{\text {low }}$ & $E_{\text {high }}$ & $E_{\text {average }}$ \\
\hline 0 & 0.0141 & 0.0200 & 0.0171 \\
1 & 0.0200 & 0.0283 & 0.0241 \\
2 & 0.0283 & 0.0400 & 0.0341 \\
3 & 0.0400 & 0.0566 & 0.0613 \\
4 & 0.0566 & 0.0800 & 0.0683 \\
5 & 0.0800 & 0.1131 & 0.0966 \\
6 & 0.1131 & 0.1600 & 0.1366 \\
7 & 0.1600 & 0.2263 & 0.1931 \\
8 & 0.2263 & 0.3200 & 0.2731 \\
9 & 0.3200 & 0.4525 & 0.3863 \\
10 & 0.4525 & 0.6400 & 0.5463 \\
11 & 0.6400 & 0.9051 & 0.7725 \\
12 & 0.9051 & 1.2800 & 1.0925 \\
13 & 1.2800 & 1.8102 & 1.5451 \\
14 & 1.8102 & 2.5600 & 2.1851 \\
15 & 2.5600 & 3.6204 & 3.0902 \\
16 & 3.6204 & 5.1200 & 4.3702 \\
17 & 5.1200 & 7.2408 & 6.1804 \\
18 & 7.2408 & 10.2400 & 8.7404 \\
19 & 10.2400 & 14.4815 & 12.3608 \\
20 & 14.4815 & 20.4800 & 17.4807 \\
\hline
\end{tabular}

Figure 28 shows the calculated energy dependence of (2) for ${ }^{4} \mathrm{He}$ in the SIS-A telescope. The filled circles in the figure show measured track widths for alphas and the FM sunward facing telescope. The figure shows key properties of the mass resolution and SIS performance:

- At energies below $\sim 250 \mathrm{keV} \mathrm{n}^{-1}$ the mass resolution is limited by the performance of the solid-state detector, which consists of its FWHM noise, and in addition at the lowest energies, the FWHM increases due to non-ionizing energy losses (not included in Fig. 28).

- At energies above $\sim 1 \mathrm{MeV} \mathrm{n}^{-1}$, the timing measurement limits the mass resolution.

- The path length contribution to the mass resolution is small compared to other elements and can therefore be neglected.

In the low- and high-energy ranges, the $\sigma_{\mathrm{E}} / E$ or $2 \sigma_{\tau} / \tau$ terms respectively dominate the mass resolution, therefore to achieve $\sigma_{\mathrm{m}} / m \sim 0.02$ these individual terms must meet the criterion $\sigma_{\mathrm{E}} / E$ and/or $2 \sigma_{\tau} / \tau \sim 0.02$ (i.e. $\sigma_{\tau}<0.01 \tau, \sigma_{\mathrm{E}}<$ $0.02 E$ ). We note that this high resolution is not possible over the entire energy range of SIS; however, it is achievable for oxygen roughly between $150 \mathrm{keV} \mathrm{n}^{-1}$ and $1.5 \mathrm{MeV} \mathrm{n}^{-1}$. Figure 29 shows $\sigma_{\mathrm{m}} / m$ over the full mass and energy range.

\subsubsection{Efficiency}

Secondary electron yields for ions passing through thin foils roughly follow the $\mathrm{d} E / \mathrm{d} x$ Bragg curve of the particles, and therefore depend on species and energy. For $\mathrm{H}$ and $\mathrm{He}$ the forward secondary electron yields peak at roughly 3-20 electrons, while for backward emission the emission is lower by a factor of approximately two. An additional important effect is the dead area of the MCP front surfaces, which is approximately $50 \%$. The triggering efficiency for single electrons hitting a channel may be less than unity since the plate bias is set to minimise ion feedback. Since both a Start and Stop pulse are required for a TOF trigger, the overall efficiency is the product of the

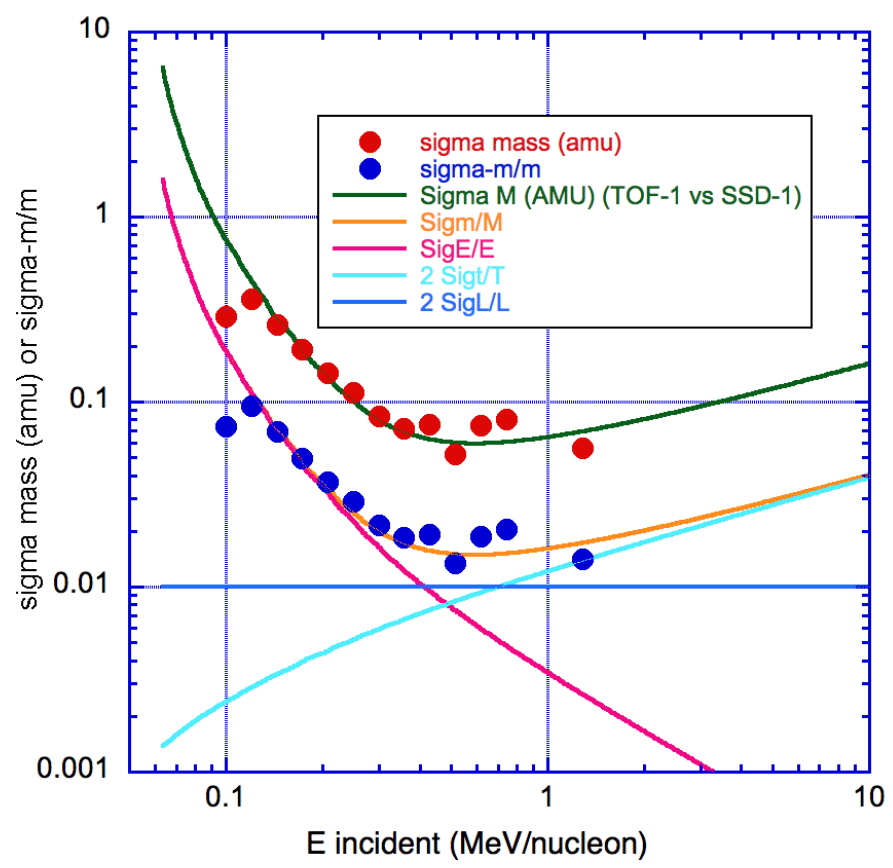

Fig. 28. Suprathermal Ion Spectrograph mass resolution contributions for ${ }^{4} \mathrm{He}$. Data points are calibration data for alphas and the FM sunward facing telescope.

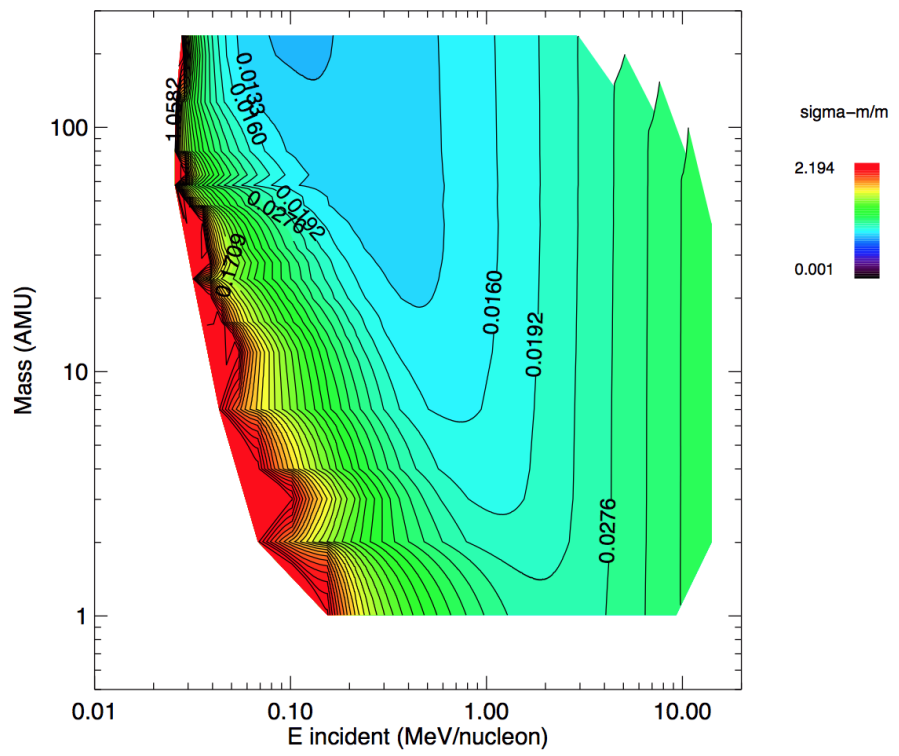

Fig. 29. Suprathermal Ion Spectrograph mass resolution as a function of species and energy.

two individual efficiencies. An estimation of the initial SIS triggering efficiency for several species is shown in Fig. 30. It has been obtained taking as basis the flight performance of the STEREO/IMPACT/SIT instrument (Mason et al. 2008).

A very low triggering efficiency for SIS for protons is acceptable since the EPD suite has other instruments that measure lowenergy protons. The alpha efficiency is also somewhat low, but is correctable after inter-calibrating with other sensors. The SIS TOF triggering efficiencies are set to give $\sim 80 \%$ triggering efficiency for alphas near $800 \mathrm{keV} \mathrm{n}^{-1}$, in order to keep the MCP signal from causing erroneous measurements for heavy ions where the signals are much larger. The MCP triggering efficiency is expected to change in flight due to (a) aging of the plates, 


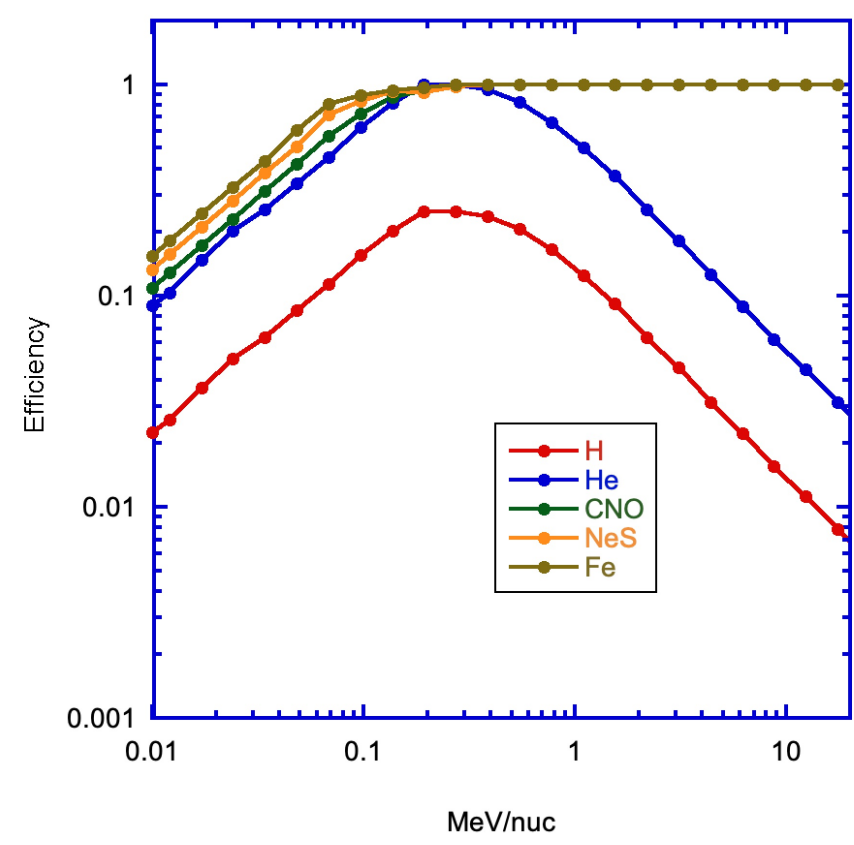

Fig. 30. Calculated initial SIS triggering efficiencies based on flight performance of the STEREO/IMPACT/SIT instrument.

(b) change in HV bias level (to compensate for changes), and (c) temperature.

\subsubsection{Calibration of the Suprathermal Ion Spectrograph}

Several calibration campaigns have been carried out on the different SIS units. This includes using the Lawrence Berkeley Laboratory 88 inch cyclotron facility. For the flight model, calibration tests have been performed with a ${ }^{241} \mathrm{Am}$ radioactive source in the Accelerator facilities at APL during 2016. The measured mass resolution for ${ }^{4} \mathrm{He}$ is $\sigma_{\mathrm{m}} / m<0.02$ at $1 \mathrm{MeV} \mathrm{n}^{-1}$ incident energy (Fig. 28).

\section{The High-Energy Telescope (HET)}

\subsection{Introduction}

The HET measures electrons, protons, and heavy ions and covers the upper energy end of the EPD range. The specific energy range depends on the species and is listed in Table 2. The HET will perform the measurements needed to understand the origin of highenergy events at the Sun which occasionally accelerate particles to such high energies that they can penetrate the atmosphere of the Earth causing a GLE. Furthermore, HET allows separation of helium isotopes down to a ${ }^{3} \mathrm{He} /{ }^{4} \mathrm{He}$ isotope ratio of about $1 \%$.

\subsection{Description of the High-Energy Telescope}

\subsubsection{Operating principles and telescope description}

The HET consists of a double-sided telescope head and an electronics box which is shared with EPT. The block diagram for EPT-HET is shown in Fig. 16. There are two EPT-HET units on the spacecraft, one located on the $-y$-deck pointing sun/antisunward along the average Parker spiral, and the other located on the $+y$-deck pointing out of the ecliptic. Thus, HET has a total of four viewing directions due to its fully symmetric design. A cross section and a mechanical drawing of the HET

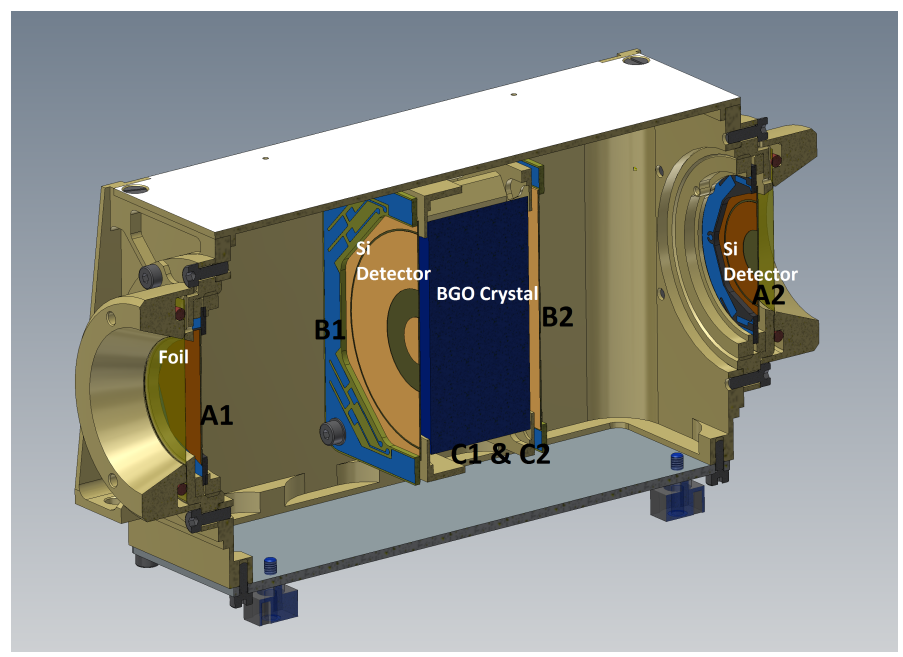

Fig. 31. Cross-section of HET sensor head.
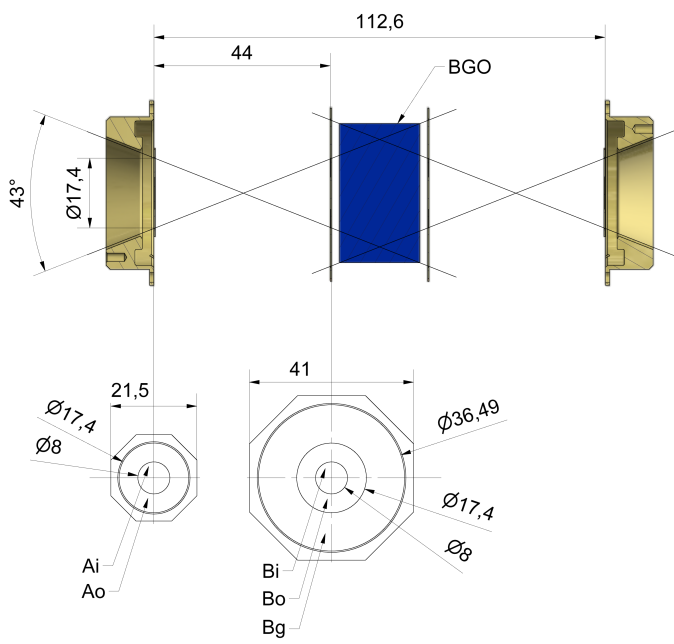

Fig. 32. Mechanical drawing of the HET telescope head. Ai and Ao are combined to $\mathrm{A}$ and $\mathrm{Bi}$ and $\mathrm{Bo}$ to $\mathrm{B}$ in the nomenclature. This is important for understanding the level-2 trigger classes in Table 20.

detector head are shown in Figs. 31 and 32, respectively. The HET entrance collimator is protected by a $50.8 \mu \mathrm{m}$ thick Kapton foil with a $25.4 \mu \mathrm{m}$ thick aluminum coating that reduces the low-energy particle flux on the front detectors. The HET consists of four $300 \mu \mathrm{m}$ thick silicon solid state detectors (SSDs) and one high-density scintillation crystal. A1 and A2 are front/back SSDs with two segments each. B1 and B2 are middle SSDs with three segments each. The naming scheme and dimensions of the SSD segments are given in Fig. 32. The scintillation detector, $\mathrm{C}$, used in HET is a hexagonal BGO $\left(\mathrm{Bi}_{4} \mathrm{Ge}_{3} \mathrm{O}_{12}\right)$ crystal of $2 \mathrm{~cm}$ in thickness which is read out by two Hamamatsu S3590-09 photodiodes. Two layers of Millipore and several layers of PTFE tape are used to avoid loss of scintillation light. Geometric factors of HET are calculated analytically using the method given in Sullivan (1971) for two circular detectors. The values are listed in Table 19. Energy and ion dependent geometric factors are calculated using Geant4 simulations.

\subsubsection{Event analysis and particle identification}

The HET and EPT units share a common electronics box and the description given for the EPT electronics in Sect. 5.2.2 also 
Table 19. Analytically calculated geometrical Factors of HET.

\begin{tabular}{lc}
\hline \hline Cases & Geometrical factor $\left(\mathrm{mm}^{2} \mathrm{sr}\right)$ \\
\hline $\mathrm{A} 1 \wedge \mathrm{B} 1$ & 27.11 \\
$\mathrm{~A} 1 \wedge \mathrm{B} 1 \wedge \mathrm{C} \wedge \mathrm{B} 2 \wedge \mathrm{A} 2$ & 4.43 \\
$\mathrm{~B} 1 \wedge \mathrm{C} \wedge \mathrm{B} 2$ & 79.36 \\
$\mathrm{~A} 1 \mathrm{i} \wedge \mathrm{B} 1$ & 1.25 \\
\hline
\end{tabular}

Notes. The first column lists the detector elements crossed in each case. Calculation based on equations given in Sullivan (1971).

applies to HET. To achieve a pulse-height 16-bit dynamic range for HET, all detectors but the Bg segments are read out by two shapers instead of one. The analysis of events itself and successive particle classification and identification in HET is performed in three levels, the so-called triggers. The level-1 trigger is a simple pulse-height-to-threshold comparison for individual detectors. The level-2 trigger requires coincidence or anti-coincidence combinations of various level-1 triggers. Table 20 summarises the level-2 triggers of HET. The numbering of these level-2 triggers is shared with EPT, thus starting at \#2. Each level-2 trigger is mapped to one of four distinct level-3 trigger classes, which determine which section of the final level-3 trigger engine (Sect. 7.2.5) will be executed.

\subsubsection{Sample High-Energy Telescope event}

In this section, the complex HET trigger logic and data processing will be illustrated using a sample oxygen particle and Fig. 33 for illustration. All single events for EPT-HET are continuously sampled and compared to a defined threshold for certain detectors. The sample oxygen particle enters the telescope housing through the entrance foil with a primary energy of $238 \mathrm{MeV}$. It deposits $190.8 \mathrm{MeV}$ in $\mathrm{A} 1 \mathrm{i}$ and $20.6 \mathrm{MeV}$ in B1o. The defined level-1 thresholds are $50 \mathrm{keV}$ for all $\mathrm{A}$ and B detectors of HET in times of low particle flux. Therefore the sample event triggers the level-1 trigger for the A1 and B1 detectors. The purpose of the level-2 trigger is a pre-classification of events regarding direction and classification in stopping or penetrating events by coincidence and anti-coincidence checks of individual detectors. Table 20 gives an overview of the HET level-2 trigger classes. The event matches the level-2 trigger \#4 and is therefore further processed by the level- 3 trigger class 1 . The level-3 trigger performs a full calibration of necessary detectors and further classifies the event in for example stopping in B or stopping in C. Each level-3 trigger performs several threshold checks and comparisons in order to optimise the output of the trigger. Since the sample event did not deposit energy in the $\mathrm{C}$ detector, the stopping level-3 trigger treats this as an $\mathrm{ABnC}$ event. A path length correction for the A detector is performed and is used to calculate $(A+B) \cdot A^{0.75}$. Based on the resulting value, the event is stored in the appropriate histogram. In this case, with a value of $10776 \mathrm{MeV}^{1.75}$, it is stored in the 2D heavy ion histogram for particles coming from the A1-, B1-side. Because of its position in this histogram it is contributing to the second data product bin of oxygen which is created with a cadence of $300 \mathrm{~s}$.

\subsubsection{Data products}

- PHA data: HET provides several classes for individual PHA events. For these events the energy deposition in all detectors is stored for analysis. These data products can be used for the verification of the nominal data products and the unit calibration. The PHA event classes are listed in Table 21.

- Nominal data products: The HET produces histograms for different particle species and energies with its level-3 trigger. The HET nominal data products are summarised in Table 22 containing energy ranges, bin numbers, and cadences.

- Low-latency data products: Low-latency data products are described in Table 23.

- Housekeeping data: Housekeeping data includes unit information like voltages and temperatures.

\subsubsection{High-Energy Telescope level-3 trigger}

The output of the level-3 triggers of HET are predefined histograms from which certain bins are combined into data products. This subsection provides a brief explanation of the output of the level-3 triggers of the HET. An example of the HET penetrating level-3 trigger output is displayed in Fig. 34. The abundances are not scaled and only selected ions are shown. The simulation data are obtained from a Geant 4 simulation with a power-law spectrum with an index of -1 . The data are postprocessed with a level-3 simulator which includes quenching in the BGO according to Tammen et al. (2015). The penetrating trigger demands a hit in all four silicon detectors. The directional separation of the ions is done in the histogram itself, by choosing the ratio of $\mathrm{AB} 1$ and $\mathrm{AB} 2$ as the $y$-axis, where $\mathrm{AB}$ is defined as $\min (\mathrm{A}, \mathrm{B})$. The relativistic particles are located near a ratio of one on the $y$-axis, while slower particles are located at lower or higher $y$-values depending on their incoming direction. On the $x$-axis the energy deposition in the BGO scintillator is used, because this leads to a good separation of individual ion species. The stopping triggers of HET have different kinds of outputs depending on the ion species. In order to explain how $\mathrm{ABnC}$ and $\mathrm{ABC}$ events are handled prior to storing them in histograms, Figs. 35 and 36 are used for illustration. The data displayed in these figures is simulation data obtained with a Geant4 simulation with the full HET telescope head, adjusted to abundances given in Reames (2017) with a power-law shape with an index of -3 . The ${ }^{3} \mathrm{He}$ to ${ }^{4} \mathrm{He}$ ratio is chosen to be $1 \%$, while the electron to proton ratio is $1: 1$. Quenching in the scintillator is calculated according to Tammen et al. (2015). The histograms are of the same type as the final 2D histograms of the HET level-3 triggers but are solely used for illustration. The electrons and the different ion species are classified by $y$-value cuts to store them in predefined histograms. These histograms differ in type, 1D or 2D and in their binning. There are separate histograms for both directions. The 1D histograms store the total deposited energy of a particle. The $2 \mathrm{D}$ histograms are more complex. By using the ratio of the total energy and the $\mathrm{d} E / \mathrm{d} x$ as the $x$-axis, several ion species can fit in one histogram with only a limited amount of bins in the $x$-direction. The $y$-axis is chosen such that the different ion species are aligned horizontally and can be discriminated by simple $y$-value cuts. This is accomplished by multiplying the total deposited energy TOT with the $\mathrm{d} E / \mathrm{d} x$. In case of the $\mathrm{ABnC}$ trigger $\mathrm{d} E / \mathrm{d} x$ is the energy deposited in the A detector and TOT is the sum of the energy deposition in the A and B detectors. In case of the $A B C$ trigger $\mathrm{d} E / \mathrm{d} x$ is the lower value of the deposited energies in $\mathrm{A}$ and $\mathrm{B}$ and TOT is the sum of the energy deposition in the $\mathrm{A}, \mathrm{B}$, and $\mathrm{C}$ detectors. An additional rotation is introduced by using TOT $\cdot(\mathrm{d} E / \mathrm{d} x)^{\gamma}$ instead of only TOT $\cdot(\mathrm{d} E / \mathrm{d} x)$ for the $y$-axis. The value of $\gamma$ is chosen such that ${ }^{3} \mathrm{He}$ and ${ }^{4} \mathrm{He}$ are aligned horizontally. This is necessary due to limitations of data product shapes. For the $\mathrm{ABnC}$ histograms $\gamma=0.75$ and for the ABC histograms $\gamma=1.5$. To illustrate the output and 
Table 20. HET level-2 trigger logic.

\begin{tabular}{lcccl}
\hline \hline L2 trigger \# & Sensor & Coincidence condition & L3 trigger class & Description \\
\hline 2 & HET & $B 1 \wedge B 2 \wedge \overline{A 1 \vee A 2}$ & 3 & HET Galactic cosmic rays \\
3 & Unused & Unused & Unused & N/A \\
4 & HET & $A 1 \wedge B 1 \wedge \overline{A 2 \vee B 2}$ & 1 & HET forward stopping in B1 or C \\
5 & HET & $A 1 \wedge B 1 \wedge B 2$ & 3 & HET forward penetrating \\
6 & HET & $A 2 \wedge B 2 \wedge \overline{A 1 \vee B 1}$ & 2 & HET backward stopping in B2 or C \\
7 & HET & $A 2 \wedge B 2 \wedge B 1$ & 3 & HET backward penetrating \\
\hline
\end{tabular}

Notes. The labels of the coincidences correspond to the labels of the respective detectors in HET. Detector nomenclature is given in Fig. 32 .

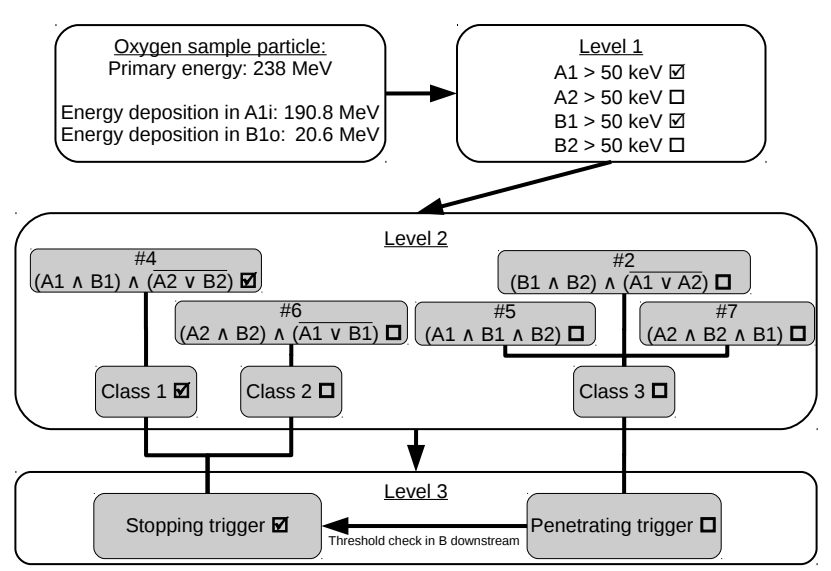

Fig. 33. Graphical illustration of an oxygen sample event illustrating the trigger logic of HET.

capability of the more complex 2D stopping histograms an example oxygen spectrum is shown for HET ABnC stopping in Fig. 37 and for HET ABC stopping in Fig. 38. The underlying simulation data are obtained using a Geant 4 simulation with a complete HET telescope head as geometry and a power law energy spectrum with an index of -1 . Based on Tammen et al. (2015) the quenching in the BGO is calculated and the data is run through a level-3 trigger simulator.

The 2D histograms on the left-hand side of Figs. 37 and 38 show the expected output of the oxygen spectrum in our level3 2D histograms. The histograms are binned as implemented on the unit. The green boxes are predefined data products, as given in Table 22 that will sum up the contents of the bins and store them in a counter. With a certain cadence this counter is then sent to Earth. The 1D histograms on the right-hand side of Figs. 37 and 38 show the primary energy in megaelectronvolts/nucleons for the ions in the displayed data product bins. The HET also features a penetrating level-3 trigger for highenergy particles. Energy ranges for this trigger are given in Table 22. In addition to the histograms that are created by the level-3 triggers, PHA words are also directly generated for stopping as well as penetrating particles. As an addition to the penetrating trigger, HET also features a GCR trigger with a high geometric factor where a coincidence of both B-detectors is required. The particles have to be detected either in the inner or outer segments of the B-detectors and may not pass through the guard ring. The energy deposition in the BGO crystal for particles fulfilling these criteria is stored in a $1 \mathrm{D}$ histogram. One nominal data product for protons with 60 s cadence and two bins will be available. In burst mode, seven bins for protons with a cadence of $1 \mathrm{~s}$ will be provided. The primary energy range for protons of this trigger starts at $107 \mathrm{MeV}$ and thus is identical to that of the penetrating level-3 trigger.
Table 21. Overview of HET PHA classes.

\begin{tabular}{ll}
\hline \hline PHA class & Description \\
\hline \multirow{3}{*}{3} & $\begin{array}{l}\text { Invalid stopping/ } \\
\text { Invalid penetrating/ } \\
\text { GCR trigger }\end{array}$ \\
\cline { 2 - 2 } 4 & Penetrating event \\
\cline { 2 - 2 } 5 & Penetrating event \\
& dep. Energy in C $>600 \mathrm{MeV}$ \\
\cline { 2 - 2 } & Heavy ions: S to Fe \\
& Stopping in C \\
6 & Stopping in B \\
11 & Heavy ions: Li to Si \\
7 & Stopping in C \\
12 & Stopping in B \\
& Helium \\
\cline { 2 - 2 } 8 & Stopping in C \\
13 & Stopping in B \\
& Proton \\
\hline 9 & Stopping in C \\
14 & Stopping in B \\
& Electrons \\
\cline { 2 - 2 } 10 & Stopping in C \\
15 & Stopping in B \\
\hline
\end{tabular}

\subsection{High-Energy Telescope operating modes}

The operational modes of the HET are the following: - Off: Off.

- Housekeeping: Only generation of housekeeping packets is enabled. Data generation is disabled.

- Operational - Low flux: Nominal operational mode with default threshold configurations and generation of nominal data products, housekeeping, and PHA data.

- Operational - High flux: Configuration for high particle flux. Threshold adjustments to reduce deadtime of the instrument as described in Sect. 7.4.

- Operational - Burst mode: Burst mode data products are continuously generated by HET and sent to the ICU: these can later be provided upon request. The burst data products are summarised in Table 24.

\subsection{High particle flux}

The geometric factors of HET can be reduced during times of high particle flux to avoid an increase in dead time. This is 
Table 22. HET nominal data products.

\begin{tabular}{|c|c|c|c|c|c|}
\hline \multirow[t]{2}{*}{ Species } & \multicolumn{2}{|c|}{$\mathrm{ABnC}$} & \multicolumn{2}{|c|}{$\mathrm{ABC}$} & \multirow{2}{*}{$\begin{array}{c}\text { Penetrating } \\
\text { 2D }\end{array}$} \\
\hline & $1 \mathrm{D}$ & $2 \mathrm{D}$ & 1D & $2 \mathrm{D}$ & \\
\hline \multicolumn{6}{|l|}{$\mathrm{e}^{-}$} \\
\hline Energy range $\left(\mathrm{MeV} \mathrm{n}^{-1}\right)$ & $0.3-1.2$ & - & $0.6-30 / 21-30$ & - & - \\
\hline Cadence (s) & 1 & - & $1 / 30$ & - & - \\
\hline \# of bins & 1 & - & $3 / 1$ & - & - \\
\hline \multicolumn{6}{|l|}{$\mathrm{H}$} \\
\hline Energy range $\left(\mathrm{MeV} \mathrm{n}^{-1}\right)$ & $6.8-10$ & - & $10-107$ & - & $100-\infty$ \\
\hline Cadence $(\mathrm{s})$ & $5 / 1$ & - & $5 / 1$ & - & 30 \\
\hline \# of bins & $5 / 1$ & - & $31 / 3$ & - & 7 \\
\hline \multicolumn{6}{|l|}{${ }^{3} \mathrm{He}$} \\
\hline Energy range $\left(\mathrm{MeV} \mathrm{n}^{-1}\right)$ & $8.1-10$ & $8.0-10$ & - & $13-41$ & - \\
\hline Cadence (s) & 5 & 30 & - & 30 & - \\
\hline \# of bins & 6 & 4 & - & 5 & - \\
\hline \multicolumn{6}{|l|}{${ }^{4} \mathrm{He}$} \\
\hline Energy range $\left(\mathrm{MeV} \mathrm{n}^{-1}\right)$ & $6.9-9.5$ & $6.9-10$ & - & $11-105$ & $100-\infty$ \\
\hline Cadence (s) & 5 & 30 & - & 30 & 60 \\
\hline \# of bins & 6 & 4 & - & 11 & 7 \\
\hline \multicolumn{6}{|l|}{$\mathrm{C}, \mathrm{N}, \mathrm{O}$} \\
\hline Energy range $\left(\mathrm{MeV} \mathrm{n}^{-1}\right)$ & - & $12-19$ & - & $21-236$ & $200-\infty$ \\
\hline Cadence (s) & - & 60 & - & 60 & 600 \\
\hline \# of bins & - & $3 \times 5$ & - & $3 \times 12$ & 10 \\
\hline \multicolumn{6}{|l|}{$\mathrm{Ne}, \mathrm{Mg}, \mathrm{Si}, \mathrm{S}$} \\
\hline Energy range $\left(\mathrm{MeV} \mathrm{n}^{-1}\right)$ & - & $16-26$ & - & $28-360$ & $370-\infty$ \\
\hline Cadence (s) & - & 300 & - & 300 & 3600 \\
\hline \# of bins & - & $4 \times 5$ & - & $4 \times 11$ & 7 \\
\hline \multicolumn{6}{|l|}{$\mathrm{Ar}, \mathrm{Ca}, \mathrm{Fe}, \mathrm{Ni}$} \\
\hline Energy range $\left(\mathrm{MeV} \mathrm{n}^{-1}\right)$ & - & $20-34$ & - & $35-500$ & $450-\infty$ \\
\hline Cadence (s) & - & 300 & - & 300 & 600 \\
\hline \# of bins & - & $4 \times 5$ & - & $4 \times 11$ & Fe: 7 \\
\hline
\end{tabular}

Notes. The ${ }^{3} \mathrm{He}$ and ${ }^{4} \mathrm{He}$ is a shared 1D histogram for outer-outer segment coincidences. ${ }^{3} \mathrm{He}$ and ${ }^{4} \mathrm{He} 2 \mathrm{D}$ histogram is only non-outer-outer segment coincidences.

Table 23. Low-latency data products of the HET.

\begin{tabular}{|c|c|c|c|c|c|}
\hline \multirow[t]{2}{*}{ Species } & \multicolumn{2}{|c|}{$\mathrm{ABnC}$} & \multicolumn{2}{|c|}{$\mathrm{ABC}$} & \multirow{2}{*}{$\begin{array}{c}\text { Penetrating } \\
\text { 2D }\end{array}$} \\
\hline & 1D & $2 \mathrm{D}$ & 1D & $2 \mathrm{D}$ & \\
\hline \multicolumn{6}{|l|}{$\mathrm{e}^{-}$} \\
\hline Energy range $\left(\mathrm{MeV} \mathrm{n}^{-1}\right)$ & $0.3-1.2$ & - & $0.6-30 / 0.8-11$ & - & - \\
\hline Cadence (s) & 30 & - & $30 / 5$ & - & - \\
\hline \# of bins & 1 & - & $3 / 1$ & - & - \\
\hline \multicolumn{6}{|l|}{$\mathrm{H}$} \\
\hline Energy range $\left(\mathrm{MeV} \mathrm{n}^{-1}\right)$ & $6.8-10$ & - & $10-107 / 13-107$ & - & $100-\infty$ \\
\hline Cadence (s) & 30 & - & $30 / 5$ & - & 30 \\
\hline \# of bins & 2 & - & $10 / 3$ & - & 6 \\
\hline \multicolumn{6}{|l|}{${ }^{3} \mathrm{He}$} \\
\hline Energy range $\left(\mathrm{MeV} \mathrm{n}^{-1}\right)$ & $8.1-10$ & $8.3-10$ & - & $13-41$ & - \\
\hline Cadence (s) & 300 & 300 & - & 300 & - \\
\hline \# of bins & 4 & 2 & - & 4 & - \\
\hline \multicolumn{6}{|l|}{${ }^{4} \mathrm{He}$} \\
\hline Energy range $\left(\mathrm{MeV} \mathrm{n}^{-1}\right)$ & $6.9-9.5$ & $7.1-8.8$ & - & $11-105$ & $100-\infty$ \\
\hline Cadence (s) & 300 & 300 & - & 300 & 300 \\
\hline \# of bins & 4 & 2 & - & 8 & 6 \\
\hline \multicolumn{6}{|l|}{$\mathrm{C}, \mathrm{N}, \mathrm{O}$} \\
\hline Energy range $\left(\mathrm{MeV} \mathrm{n}^{-1}\right)$ & - & $13-19$ & - & $30-236$ & $200-\infty$ \\
\hline Cadence (s) & - & 600 & - & 600 & 3600 \\
\hline \# of bins & - & $3 \times 2$ & - & $3 \times 3$ & 6 \\
\hline \multicolumn{6}{|l|}{$\mathrm{Fe}$} \\
\hline Energy range $\left(\mathrm{MeV} \mathrm{n}^{-1}\right)$ & - & $23-33$ & - & $82-470$ & $500-630$ \\
\hline Cadence (s) & - & 600 & - & 3600 & 3600 \\
\hline \# of bins & - & 2 & - & 3 & 2 \\
\hline
\end{tabular}

Notes. The ${ }^{3} \mathrm{He}$ and ${ }^{4} \mathrm{He}$ is a shared $1 \mathrm{D}$ histogram for outer-outer segment coincidences. ${ }^{3} \mathrm{He}$ and ${ }^{4} \mathrm{He} 2 \mathrm{D}$ histogram is only non-outer-outer segment coincidences. 


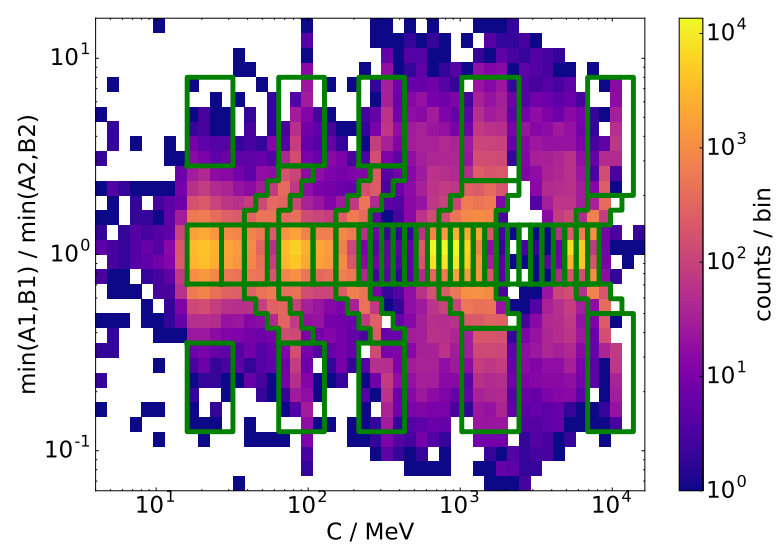

Fig. 34. Histogram of the level-3 penetrating trigger of HET with unitlike binning and data product boxes created with simulation data for $\mathrm{H}$, $\mathrm{He}, \mathrm{C}, \mathrm{N}, \mathrm{O}$ and $\mathrm{Fe}$. The letters $\mathrm{A}, \mathrm{B}$, and $\mathrm{C}$ denote the energy deposition in the respective detectors $\mathrm{A}, \mathrm{B}$, and $\mathrm{C}$.

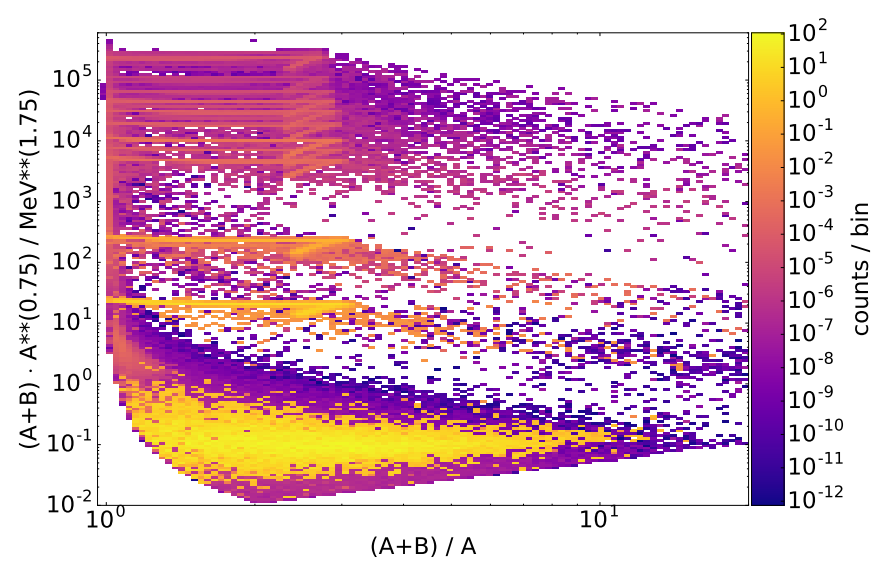

Fig. 35. Illustration for the HET level-3 ABnC trigger containing scaled simulation data. The letters A and B denote the energy deposition in the respective SSDs A and B.

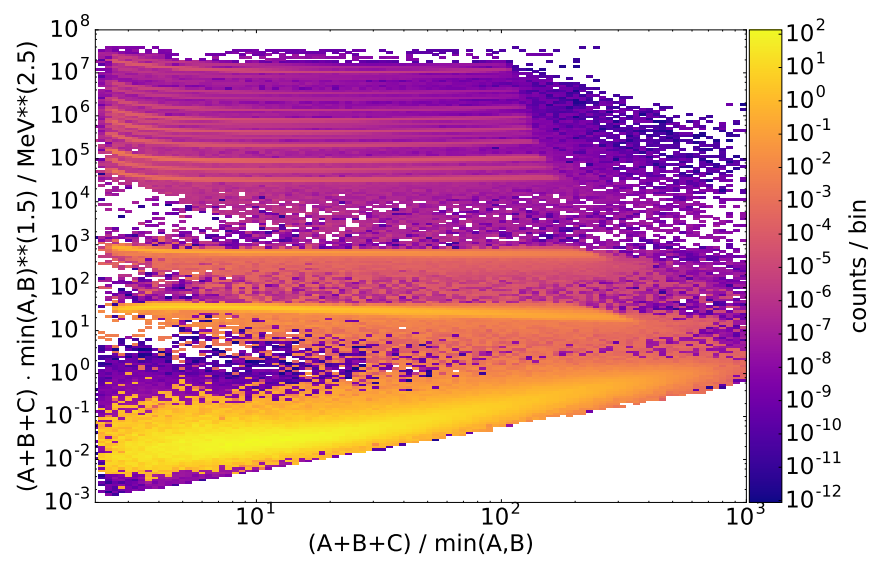

Fig. 36. Illustration for the HET level-3 ABC trigger containing scaled simulation data. The letters $\mathrm{A}, \mathrm{B}$, and $\mathrm{C}$ denote the energy deposition in the respective detectors $\mathrm{A}, \mathrm{B}$, and $\mathrm{C}$.

achieved by raising the level-1 thresholds for the outer segments of the A-detectors to $6.2 \mathrm{MeV}$ such that electrons and protons are fully rejected for all HET triggers. Particles entering the telescope through the inner segments of A are not affected by these cuts. The geometric factor reduction is given in Table 19. The effect of raising the threshold of the A-detectors outer segment is illustrated for the ABnC trigger in Fig. 39 with simulation data. In the $\mathrm{ABnC}$ trigger only protons and electrons are affected. For the $\mathrm{ABC}$ and penetrating trigger the threshold increase affects also heavier ions. The reduced energy range for the affected ions in the ABC trigger are $19.3 \mathrm{MeV} \mathrm{n}^{-1}$ for ${ }^{3} \mathrm{He}$ and $20 \mathrm{MeV} \mathrm{n}^{-1}$ for ${ }^{4} \mathrm{He}$. For the penetrating trigger the energy range of $\mathrm{C}$ is reduced to $410 \mathrm{MeV} \mathrm{n}^{-1}$, the range for $\mathrm{N}$ to $860 \mathrm{MeV} \mathrm{n}^{-1}$ and oxygen is unaffected. All lighter ions than $\mathrm{C}$ are fully rejected by the increased threshold in the outer segment of the A-detector.

\subsection{Calibration of the High-Energy Telescope}

The HET FM units were calibrated in a thermal vacuum chamber. The FM1 was calibrated in a temperature range between $-17^{\circ} \mathrm{C}$ up to $+46^{\circ} \mathrm{C}$ in seven temperature steps and FM 2 between $-7^{\circ} \mathrm{C}$ up to $+46^{\circ} \mathrm{C}$ in six temperature steps. The SSDs were calibrated using X-rays and resulting Compton edges from a ${ }^{207} \mathrm{Bi}$ radioactive source that was placed adjacent to the unit. Only single detector hits were used to ensure the detection of X-rays only. SSD calibration was performed using a group of five Xray peaks that are modelled with Gaussian distributions for the fit. A Compton edge of the $569.698 \mathrm{keV} \gamma$-line is modelled with an inverse error function. The energies used for calibration are given in Table 25. For the calibration of the scintillation detector cosmic muons were used which were fitted with an approximation of a Landau distribution. The instrument was mounted vertically in the chamber to allow coincidence detection of muons in all HET SSDs and the scintillation detector. The deposited energy of $18.024 \mathrm{MeV}$ of the cosmic muons was calculated using a Geant 4 simulation. For each temperature step the individual peak positions were determined by fitting. From the fit results a temperature correction model for the SSDs, the crystal, and low-to-high-gain conversion factors was developed. The calibration factors given in Table 26 for FM1 and in Table 27 for FM2 are normalised to $0^{\circ} \mathrm{C}$. The temperature correction model used for all HET detectors and low-to-high-gain correction is given in Eq. (3). The parameters used for the individual detectors are given in Table 28 for HET FM1 and in Table 29 for HET FM2.

$$
f(T)=\frac{1}{m \cdot T^{2}+n \cdot T+1} .
$$

\subsubsection{Temperature dependence of the High-Energy Telescope}

The light output of most inorganic scintillators, including BGO, is dependent on temperature (Weber et al. 2003). The temperature dependence of BGO itself is well known and was studied in detail by Melcher et al. (1985). Their explanation for the temperature dependence is attributed to thermal excitations of the electrons to radiationless transitions. With increasing temperature the probability of a radiationless de-excitation of electrons increases, resulting in a reduced light output at high temperatures (Melcher et al. 1985). Figure 40 shows the temperature dependence of the scintillation efficiency of a BGO scintillator.

The scintillation crystal in HET is read out by two Hamamatsu PIN photodiodes which are glued to the crystal using a transparent, rubber-like silicone glue. Experiments performed with an engineering model at different temperatures at a heavy ion accelerator (HIMAC, Japan) revealed that this scintillation light detection technique is sensitive to the crystal temperature. This effect is attributed to a contraction of the glue at low temperatures which leads to a detachment of the glue from the 

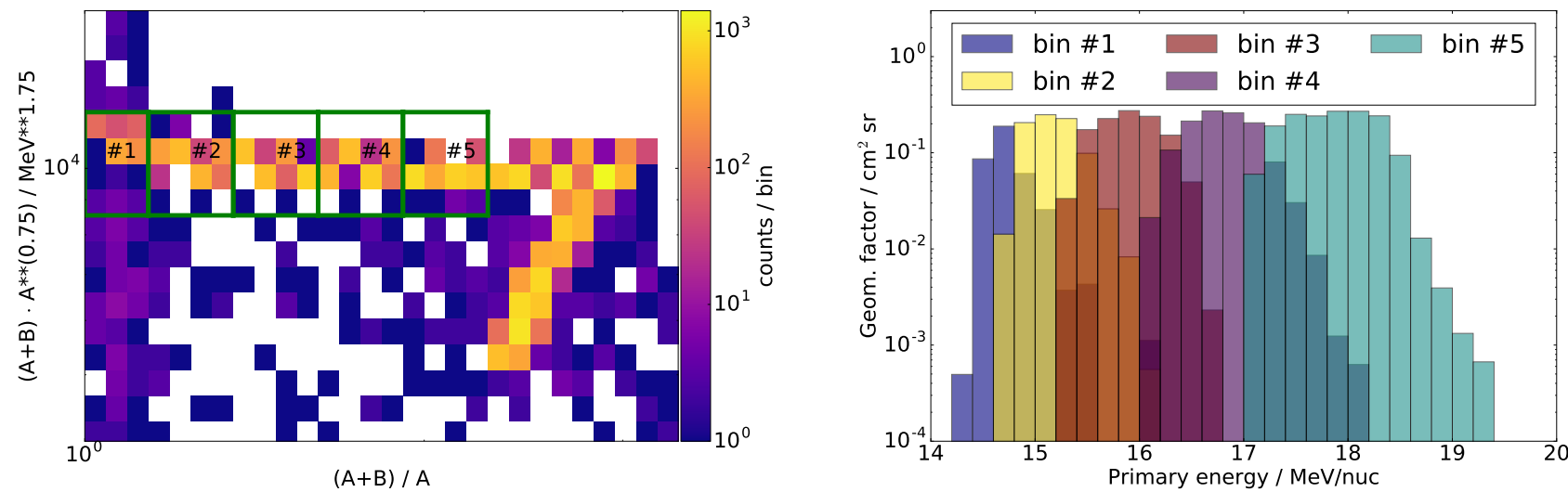

Fig. 37. Simulated output of the HET ABnC level-3 trigger for oxygen ions. Left-hand side: 2D histogram shows the level-3 trigger histogram including data product bins. Right-hand side: 1D histogram shows the energy range and geometric factor of these bins. The letters A and B denote the energy deposition in the respective SSDs A and B.
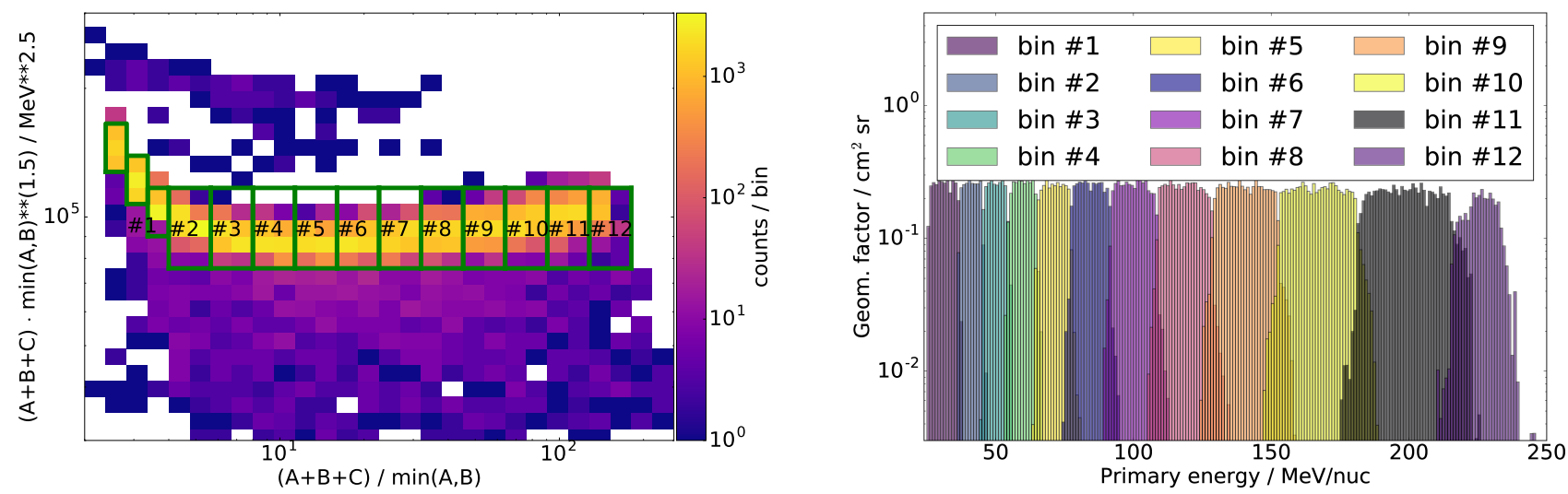

Fig. 38. Simulated output of the HET ABC level-3 trigger for oxygen ions. Left-hand side: 2D histogram shows the level-3 trigger histogram including data product bins. Right-hand side: 1D histogram shows the energy range and geometric factor of these bins. The letters A, B, and C denote the energy deposition in the respective detectors A, B, and C.

Table 24. Burst data products of the HET.

\begin{tabular}{|c|c|c|c|c|c|}
\hline \multirow[t]{2}{*}{ Species } & \multicolumn{2}{|c|}{$\mathrm{ABnC}$} & \multicolumn{2}{|c|}{$\mathrm{ABC}$} & \multirow{2}{*}{$\begin{array}{c}\text { Penetrating } \\
\text { 2D }\end{array}$} \\
\hline & $1 \mathrm{D}$ & $2 \mathrm{D}$ & $1 \mathrm{D}$ & $2 \mathrm{D}$ & \\
\hline \multicolumn{6}{|l|}{$\mathrm{e}^{-}$} \\
\hline Energy range $\left(\mathrm{MeV} \mathrm{n}^{-1}\right)$ & $0.3-1.2$ & - & $0.6-30$ & - & - \\
\hline Cadence (s) & 1 & - & 1 & - & - \\
\hline \# of bins & 1 & - & 3 & - & - \\
\hline \multicolumn{6}{|l|}{$\mathrm{H}$} \\
\hline Energy range $\left(\mathrm{MeV} \mathrm{n}^{-1}\right)$ & $6.8-10$ & - & $10-107$ & - & $100-\infty$ \\
\hline Cadence (s) & 1 & - & 1 & - & 1 \\
\hline \# of bins & 6 & - & 62 & - & 7 \\
\hline \multicolumn{6}{|l|}{${ }^{3} \mathrm{He}$} \\
\hline Energy range $\left(\mathrm{MeV} \mathrm{n}^{-1}\right)$ & $8.1-10$ & $8.0-10$ & - & $13-41$ & - \\
\hline Cadence (s) & 1 & 5 & - & 5 & - \\
\hline \# of bins & 4 & 4 & - & 5 & - \\
\hline \multicolumn{6}{|l|}{${ }^{4} \mathrm{He}$} \\
\hline Energy range $\left(\mathrm{MeV} \mathrm{n}^{-1}\right)$ & $6.9-9.5$ & $6.9-10$ & - & $11-105$ & $100-\infty$ \\
\hline Cadence (s) & 1 & 5 & - & 5 & 5 \\
\hline \# of bins & 4 & 4 & - & 11 & 7 \\
\hline
\end{tabular}

Notes. The ${ }^{3} \mathrm{He}$ and ${ }^{4} \mathrm{He}$ is a shared 1D histogram for outer-outer segment coincidences. ${ }^{3} \mathrm{He}$ and ${ }^{4} \mathrm{He} 2 \mathrm{D}$ histogram is only non-outer-outer segment coincidences. 




Fig. 39. Black line: high particle flux cut for the HET ABnC trigger.

Table 25. Calibration of HET SSDs was performed using these energies.

\begin{tabular}{lcc}
\hline \hline Type & Energy $(\mathrm{keV})$ & Intensity \\
\hline$\gamma$ & 72.805 & $21.4 \%$ \\
$\gamma$ & 74.969 & $35.7 \%$ \\
$\gamma$ & 84.45 & $4.31 \%$ \\
$\gamma$ & 84.938 & $8.27 \%$ \\
$\gamma$ & 87.3 & $30.2 \%$ \\
Compton edge & 393.306 & - \\
\hline
\end{tabular}

Notes. Energies and intensities taken from NuDat (2011).

silicon of the photodiode, creating an optical barrier. The amount of lost light is dependent on the individual photodiode as shown in Fig. 41 where the amount of detected light of photodiode \#2 is drastically reduced below $20^{\circ} \mathrm{C}$ compared to photodiode \#1. The effect is fully reversible and disappears above a certain temperature when the glue is again attached to the silicon. Because the temperature dependence of the light coupling might be different for each photodiode, each flight unit has been calibrated individually as presented in Sect. 7.5. An in-flight calibration of HET is also possible with the provided PHA-data (Sect. 7.2.4).

\subsubsection{Quenching in the $\mathrm{Bi}_{4} \mathrm{Ge}_{3} \mathrm{O}_{12}$ crystal}

Most scintillators, including BGO, show a non-linearity in light output for heavy ionizing particles. The light loss is dependent on the scintillator material, the particle type and its energy. The effect is know as ionization quenching and was empirically described by J. B. Birks (Birks \& Firk 1965). Birks law (4) describes the light output $\mathrm{d} L$ per unit length $\mathrm{d} x$, with a scintillation efficiency $S$ and the Birks constant $k_{\mathrm{B}}$, which is a material specific quenching factor. The effect of quenching is illustrated in Fig. 42. The right hand side of the plot shows experimental data taken at the Heavy Ion Accelerator in Chiba (HIMAC), Japan, with $400 \mathrm{MeV} \mathrm{n}^{-1}$ carbon ions measured by an HET EM. The left hand side of Fig. 42 shows the corresponding Geant 4 simulation results. While the energy in the silicon tracking diode ( $y$-axis) is in good agreement when comparing experiment and simulation, the measured energy in the BGO crystal is clearly not.
Table 26. Calibration factors for all HET FM1 detectors/segments including pha channel numbers.

\begin{tabular}{lcccc}
\hline \hline $\begin{array}{l}\text { Channel } \\
\text { name }\end{array}$ & $\begin{array}{c}\text { HG } \\
\text { channel }\end{array}$ & $\begin{array}{c}\text { LG } \\
\text { channel }\end{array}$ & $\begin{array}{c}\text { HG calib. } \\
\left(\mathrm{keV} \mathrm{A}^{-1}\right)\end{array}$ & $\begin{array}{c}\text { LG calib. } \\
\left(\mathrm{keV} \mathrm{A}^{-1}\right)\end{array}$ \\
\hline A1i & 6 & 8 & 1.343 & 18.591 \\
A1o & 21 & 9 & 1.330 & 18.350 \\
$\mathrm{~B} 1 \mathrm{i}$ & 1 & 0 & 1.299 & 18.012 \\
$\mathrm{~B} 1 \mathrm{o}$ & 2 & 3 & 1.336 & 18.506 \\
$\mathrm{~B} 1 \mathrm{~g}$ & 7 & - & 1.132 & - \\
$\mathrm{C} 1$ & 5 & 4 & 18.785 & 261.619 \\
$\mathrm{C} 2$ & 18 & 20 & 18.785 & 261.619 \\
$\mathrm{~B} 2 \mathrm{i}$ & 11 & 10 & 1.306 & 18.364 \\
$\mathrm{~B} 2 \mathrm{o}$ & 13 & 12 & 1.307 & 17.821 \\
$\mathrm{~B} 2 \mathrm{~g}$ & 19 & - & 1.170 & - \\
$\mathrm{A} 2 \mathrm{i}$ & 16 & 17 & 1.329 & 18.148 \\
A2o & 15 & 14 & 1.366 & 18.895 \\
\hline
\end{tabular}

Notes. $\mathrm{C} 1$ and $\mathrm{C} 2$ are averaged and calibrated together, thus sharing one calibration. Calibration factors are normalised to $0^{\circ} \mathrm{C}$.

Table 27. Calibration factors for all HET FM2 detectors/segments including pha channel numbers.

\begin{tabular}{lcccc}
\hline \hline Name & $\begin{array}{c}\text { HG } \\
\text { channel }\end{array}$ & $\begin{array}{c}\text { LG } \\
\text { channel }\end{array}$ & $\begin{array}{c}\text { HG calib. } \\
\left(\mathrm{keV} \mathrm{A}^{-1}\right)\end{array}$ & $\begin{array}{c}\text { LG calib. } \\
\left(\mathrm{keV} \mathrm{A}^{-1}\right)\end{array}$ \\
\hline $\mathrm{A} 1 \mathrm{i}$ & 6 & 8 & 1.349 & 18.738 \\
$\mathrm{~A} 1 \mathrm{o}$ & 21 & 9 & 1.329 & 18.316 \\
$\mathrm{~B} 1 \mathrm{i}$ & 1 & 0 & 1.304 & 18.461 \\
$\mathrm{~B} 10$ & 2 & 3 & 1.406 & 18.933 \\
$\mathrm{~B} 1 \mathrm{~g}$ & 7 & - & 1.125 & - \\
$\mathrm{C} 1$ & 5 & 4 & 17.404 & 241.115 \\
$\mathrm{C} 2$ & 18 & 20 & 17.404 & 241.115 \\
$\mathrm{~B} 2 \mathrm{i}$ & 11 & 10 & 1.330 & 18.275 \\
$\mathrm{~B} 2 \mathrm{o}$ & 13 & 12 & 1.332 & 18.258 \\
$\mathrm{~B} 2 \mathrm{~g}$ & 19 & - & 1.173 & - \\
$\mathrm{A} 2 \mathrm{i}$ & 16 & 17 & 1.493 & 19.055 \\
$\mathrm{~A} 2 \mathrm{o}$ & 15 & 14 & 1.344 & 18.124 \\
\hline
\end{tabular}

Notes. $\mathrm{C} 1$ and $\mathrm{C} 2$ are averaged and calibrated together, thus sharing one calibration. Calibration factors are normalised to $0^{\circ} \mathrm{C}$.

For the definition of histograms and other data products we derived a model for heavy ions $(Z \geq 2)$ to predict the amount of ionization quenching which is capable of reducing the mismatch between deposited and measured energy down to below $\sim 10 \%$.

$$
\begin{aligned}
& \frac{\mathrm{d} L}{\mathrm{~d} x}=S \cdot \frac{\frac{\mathrm{d} E}{\mathrm{~d} x}}{1+k_{\mathrm{B}} \frac{\mathrm{d} E}{\mathrm{~d} x}}, \\
& \begin{aligned}
& \frac{\mathrm{d} E}{\mathrm{~d} x} \approx \frac{C \cdot A Z^{2}}{E^{0.7}} \\
& L(E, A, Z)=\int \mathrm{d} L=\int_{0}^{x_{\max }} \mathrm{d} x \frac{S \cdot \frac{\mathrm{d} E}{\mathrm{~d} x}}{1+k_{\mathrm{B}} \frac{\mathrm{d} E}{\mathrm{~d} x}} \\
&=f_{1}\left(E-f_{2} \cdot A Z^{2} \cdot \log \left(\frac{E+f_{2} \cdot A Z^{2}}{f_{2} \cdot A Z^{2}}\right)\right) .
\end{aligned}
\end{aligned}
$$

Quenching model for BGO. The heavy ion data for the quenching model was taken at HIMAC, Japan. For each measured ion several energies have been measured and compared 
Table 28. Parameters $m$ and $n$ used for the temperature correction of the calibration values given in Table 26 for HET FM1 using Eq. (3).

\begin{tabular}{lcc}
\hline \hline Temp. correction & $m\left({ }^{\circ} \mathrm{C}^{-2}\right)$ & $n\left({ }^{\circ} \mathrm{C}^{-1}\right)$ \\
\hline SSD & $-1.236 \times 10^{-5}$ & $8.845 \times 10^{-4}$ \\
SSD low- to high gain & $-1.988 \times 10^{-5}$ & $9.727 \times 10^{-4}$ \\
BGO & $1.310 \times 10^{-5}$ & $-7.869 \times 10^{-3}$ \\
BGO low- to high gain & $-1.823 \times 10^{-5}$ & $9.15 \times 10^{-4}$ \\
\hline
\end{tabular}

Table 29. Parameters $m$ and $n$ used for the temperature correction of the calibration values given in Table 27 for HET FM2 using Eq. (3).

\begin{tabular}{lcc}
\hline \hline Temp. correction & $m\left({ }^{\circ} \mathrm{C}^{-2}\right)$ & $n\left({ }^{\circ} \mathrm{C}^{-1}\right)$ \\
\hline SSD & $-3.981 \times 10^{-6}$ & $6.021 \times 10^{-4}$ \\
SSD low- to high gain & $-8.021 \times 10^{-6}$ & $3.531 \times 10^{-4}$ \\
BGO & $-3.396 \times 10^{-5}$ & $-7.486 \times 10^{-3}$ \\
BGO low- to high gain & $-1.927 \times 10^{-5}$ & $8.645 \times 10^{-4}$ \\
\hline
\end{tabular}

to the according simulated energy depositions. An example for carbon ions is shown in Fig. 43. The individual points resemble measurements with different absorber thicknesses which alter the energy of the ions. The solid line is a numerical solution of Eq. (6). This equation results from using Eq. (5) in Eq. (6). From the numerical solution two parameters, $f_{1}=S$, which is a measure of the energy to light conversion efficiency for a specific ion, and $f_{2}=C \cdot k_{\mathrm{B}}$, which describes the probability of quenching of charge carriers, are obtained for each measured ion (Tammen et al. (2015)). To predict the quenching in BGO for ions which have not been measured, a set of two Eqs. (7) and (8), is introduced. These equations are fitted to the obtained parameters for the individual ions $f_{1}$ and $f_{2}$. The resulting parameters for Eqs. (7) and (8) used in these functions are given in Table 30. Using these parameters we can predict the quenching for heavy ions which have not been measured during our study and thus reduce the mismatch between the simulations and the experiment.

$f_{1}(Z)=\min \left(I_{1}, A 1 \cdot \exp \left(-\lambda_{1} \cdot Z+O_{1}\right)\right)$,

$f_{2}(Z)=I_{2} \cdot \exp \left(-\lambda_{2} \cdot Z\right)$

\section{The Instrument Control Unit (ICU) and on-board software}

The Instrument Control Unit or ICU (Fig. 44) is the interface between the spacecraft and the EPD sensors. All EPD sensors are connected to the ICU, which provides them with a telecommand and telemetry communication link, time synchronization, processing, and power. The ICU is composed of the common data processing unit (CDPU) and the low-voltage power supply (LVPS), operating in a cold redundant configuration. Therefore the ICU contains four electronic boards, two for the CDPU (nominal+redundant) and two for the LVPS (nominal+redundant). The ICU of the EPD inherits its structure from the CDPU of the CEPAC instrument of SOHO (MüllerMellin et al. 1995). The CEPAC CDPU has been operating since the launch of SOHO without failure. The design of the CDPU follows a HW/SW co-design approach and is based on

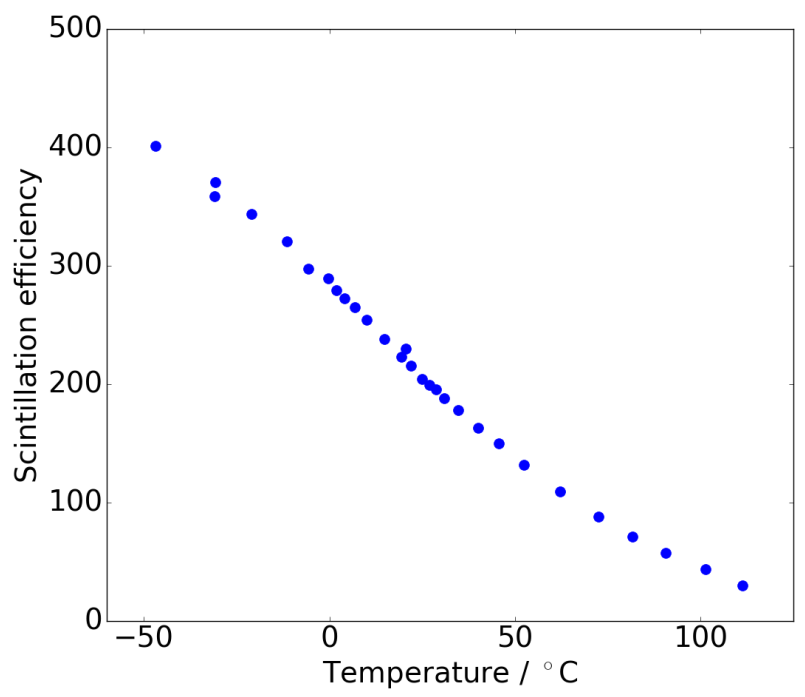

Fig. 40. Light output of BGO, excited by $662 \mathrm{keV}$ gamma rays. Data were extracted from Fig. 1 in Melcher et al. (1985). Error bars are not shown.

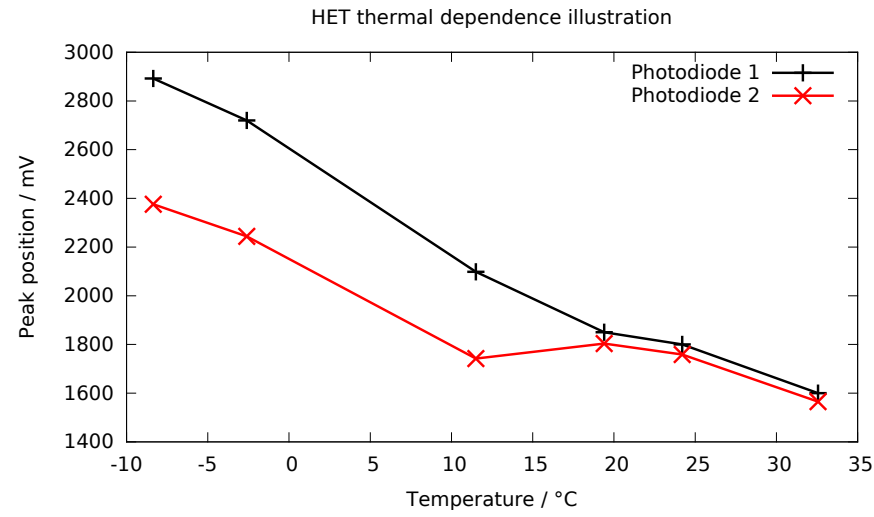

Fig. 41. Data taken at HIMAC with $600 \mathrm{MeV} \mathrm{n}^{-1} \mathrm{Ne}$ ions using a BGO FM-like scintillation crystal assembly.

a LEON2 soft-processor implemented in a RTAX2000 FPGA from Microsemi. The LVPS provides power to the CDPU and the sensors. The ICU boards are packaged in a single box in order to reduce mass and to simplify harnessing and interfaces. This box is mounted inside the spacecraft body. Electronic parts for the ICU were selected according to criteria for reliability, size, functionality, radiation hardness, and low power consumption. Parts were specified to be tested to achieve a minimum total dose radiation tolerance requirement of $100 \mathrm{krads}$. Each sensor has its own front-end electronics that communicates with the ICU through Universal Asynchronous Receiver Transmitter/LowVoltage Differential Signaling (UART/LVDS) interfaces operating at 115200 bauds. Sensors have also an interface to receive a pulse per second from the ICU. The pulse per second is generated synchronously with the reception of the SpaceWire timecode that the spacecraft transmits periodically. Sensors, as a response to this pulse, provide synchronised data to the ICU with a 1-s period, except SIS that sends data with a period of $3 \mathrm{~s}$. The ICU shares information with MAG, RPW, and SWA Solar Orbiter instruments to allow synchronised high-data-rate burstmode operations following the on-board identification of predefined triggering events in the EPD data. The internal structure of the ICU can be seen in Fig. 45. 

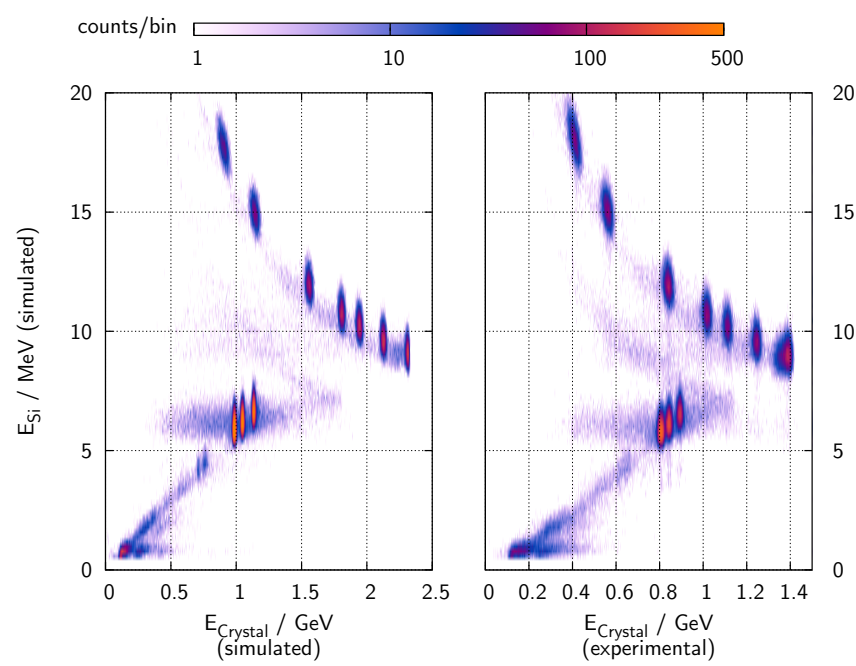

Fig. 42. Carbon ions with different energies detected by HET EM. Lefthand plot: simulation data, right-hand plot: experimental data taken at HIMAC, Japan. Tammen et al. (2015).

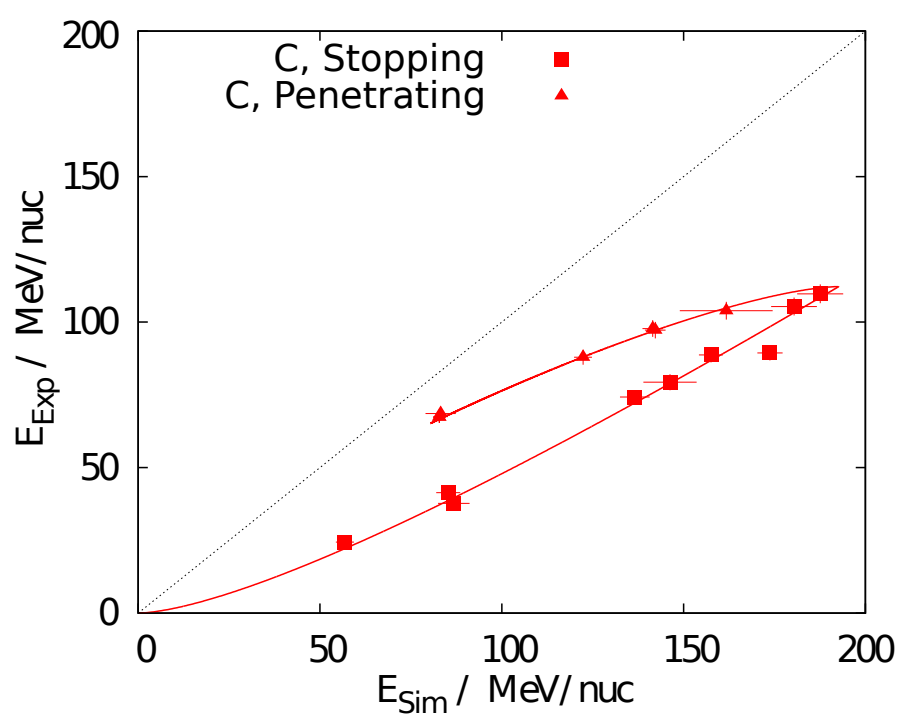

Fig. 43. Light yield of carbon for stopping and penetrating particles at different energies. The solid line is the numerical solution of Eq. (7) Tammen et al. (2015).

Table 30. Parameters found from the BGO quenching fits of Eqs. (7) and (8) to the $f_{1}$ and $f_{2}$ parameters of the individual ions.

\begin{tabular}{lc}
\hline \hline Parameter & Value \\
\hline$I_{1}$ & $1.04 \pm 0.12$ \\
$A_{1}$ & $1.109 \pm 0.077$ \\
$\lambda_{1}$ & $0.089 \pm 0.021$ \\
$O_{1}$ & $0.344 \pm 0.071$ \\
\hline$I_{2}$ & $0.85 \pm 0.20$ \\
$\lambda_{2}$ & $0.272 \pm 0.024$ \\
\hline
\end{tabular}

References. Tammen et al. (2015).

The main functions of the ICU and the on-board software are the following:

- To provide $+28 \mathrm{~V}$ power supply to the sensors. Latchcurrent limiters (LCL) are used to switch the sensors on and off

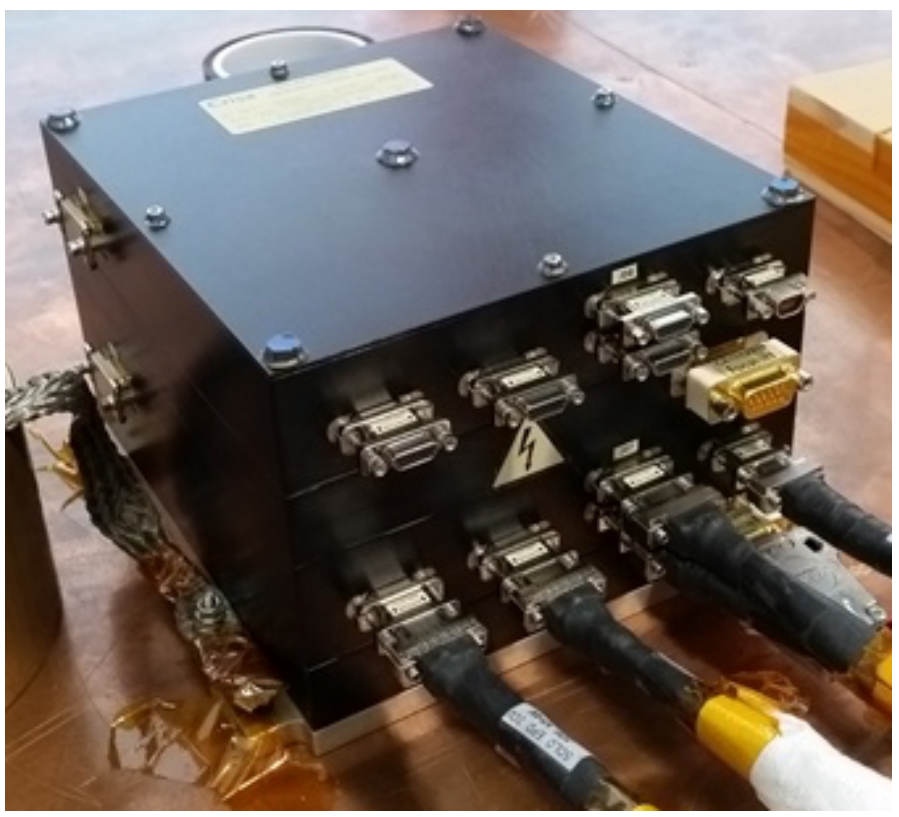

Fig. 44. Instrument Control Unit of the Energetic Particle Detector.

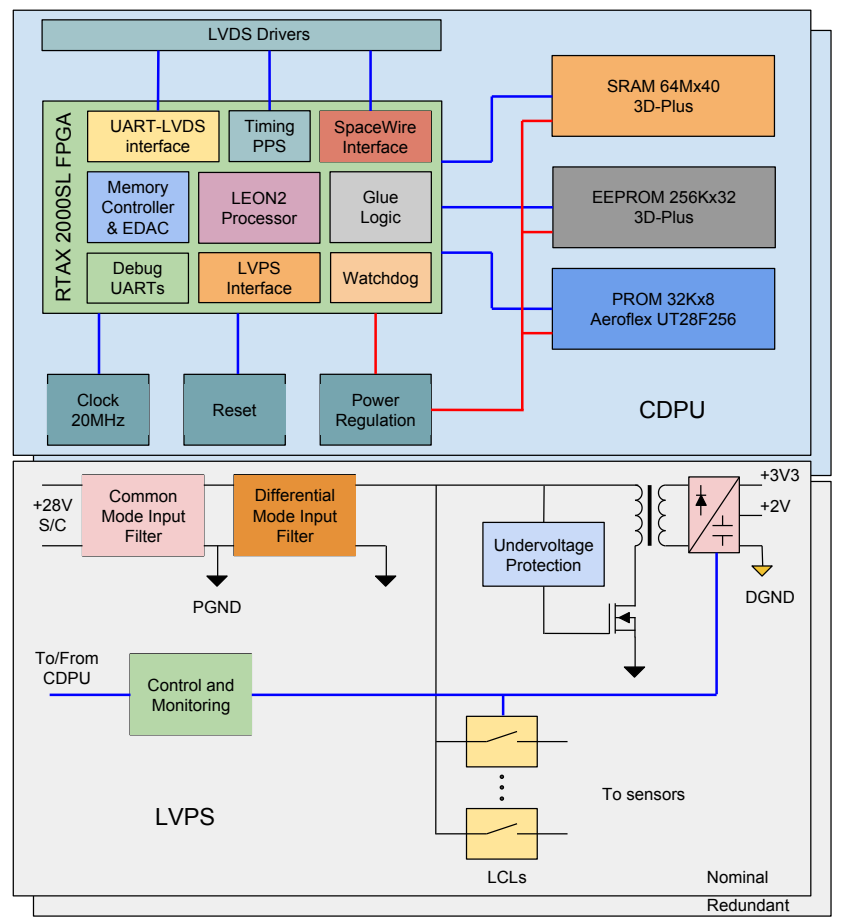

Fig. 45. Schematic of the ICU depicting the main components of the unit.

and also for monitoring current and voltage. In the case of overcurrent or under-voltage, LCLs provide protection to the sensors.

- To distribute a pulse-per-second signal synchronously with the reception of the SpaceWire time-code, or in case the latter is missing, in an autonomous way with a period of one second.

- To receive, store, and process the sensor telemetries, and generate from them the CCSDS (Consultative Comittee for Space Data Systems) science telemetries. Although the high peak baudrate of the data sent by the sensors requires the process to be completed in the background, the on-board software manages the storage always in the foreground, ensuring proper 
labelling of the absolute time at which the telemetries were received.

- To store and manage the configuration tables of the sensors. These tables are made up of configuration message sequences that can be released to the sensors as a response to a ground command, or autonomously as part of the EPD error recovery mechanism. The sequences definition includes the interval between messages, which is controlled by the on-board software.

- To provide standard PUS (Packet Utilisation Standard) services, such as telecommand verification (service 1), housekeeping (service 3 ), event reporting (service 5), memory management (service 6), on-board monitoring (service 12) or event-action (service 19).

- To manage the accepted telecommands received from the spacecraft. These telecommands are executed, or forwarded to the sensors, depending on the process to which they are addressed.

- To implement the mode and state logic required by the EPD instrument. This logic includes a safe mode, random-access memory (RAM) fault-tolerant, from which it is possible to reconfigure the EPD and replace the application software images from Earth in order to continue working in nominal mode.

- To provide a fault detection, isolation, and recovery mechanism. Examples of the errors managed by this mechanism are: application software storage corruption, double EDAC error, software execution fatal error, ICU parameter out of limits, and sensor parameter out of limits. Examples of recovery actions are: transition to safe mode, ICU reboot and switch the application software image, turn off a sensor, sensor power cycle, or the sending of a configuration message sequence to a sensor. The on-board software catches processor exceptions, and implements the PUS services 5, 12, and 19 in order to support this mechanism.

\section{Conclusions}

The EPD is the scientific instrument on-board Solar Orbiter that will study the energetic particle populations in the inner heliosphere. It has been specifically designed and developed to perform key measurements of these populations and consists of four sensors measuring energetic electrons, protons, and ions, operating at partly overlapping energy ranges covering from a few kiloelectronvolts to $450 \mathrm{MeV} \mathrm{n}^{-1}$ : STEP, consisting of one unit with two sensor heads, that will measure $2-100 \mathrm{keV}$ electrons and 3-100keV protons; EPT, consisting of two units with two sensor heads each equipped with double ended telescopes, that will measure $20-400 \mathrm{keV}$ electrons and $20 \mathrm{keV}-15 \mathrm{MeV}$ protons; SIS, consisting in one unit with two telescopes, that will measure all elements from $\mathrm{He}$ to $\mathrm{Fe}$ (sampling trans-iron elements) in the energy range $50 \mathrm{keV} \mathrm{n}^{-1}-14 \mathrm{MeV} \mathrm{n}^{-1}$ (for CNO); and HET, which consists of two units with one sensor head each that is equipped with double ended telescopes. The HET will measure electrons $(0.3-15 \mathrm{MeV})$, protons $(7-105 \mathrm{MeV})$, and ions (7-450 $\mathrm{MeV} \mathrm{n}^{-1}, Z$-dependent). The four EPD sensors share a common ICU composed of the CDPU and the LVPS; it is the sole power and data interface of EPD to the spacecraft.

The temporal resolution of EPD, of up to one second, its energy range that extends from suprathermal energies (overlapping with SWA) up to a few hundred megaelectronvolts per nucleon, its elemental coverage that identifies all species from $\mathrm{H}$ to $\mathrm{Fe}$ including isotopic resolution, and its different FoVs Sunward and AntiSunward - in ecliptic and out of the ecliptic plane are described in this paper. All these capabilities fulfil and, in some cases, exceed, the instrument requirements defined to accomplish the scientific objectives of the mission and particularly those related to the mechanisms involved in injection, acceleration, and transport of the energetic particles.

Acknowledgements. We gratefully acknowledge the hard work and dedication of the many people who contributed to EPD on Solar Orbiter at all of our institutions. It was a unique experience for all of us. We acknowledge the Spanish Ministerio de Ciencia, Innovación y Universidades for their invaluable support on the development of the EPD suite and the ICU under grants FEDER/MCIU - Agencia Estatal de Investigación/Projects ESP2105-68266-R and ESP2017-88436-R. We thank the German Federal Ministry for Economic Affairs and Energy and the German Space Agency (Deutsches Zentrum für Luft- und Raumfahrt, e.V., (DLR)) for their unwavering support of STEP, EPT, and HET under grants numbers 50OT0901, 50OT1202, and 50OT1702; and ESA for supporting the build of SIS under contract number SOL.ASTR.CON.00004, and the University of Kiel and the Land Schleswig-Holstein for their support of SIS. We also thank the ESA project team for their support and expert knowledge. We thank E. Valtonen from the University of Turku, Finland, for his inestimable contribution to EPD.

\section{References}

Afanasiev, A., Battarbee, M., \& Vainio, R. 2015, A\&A, 584, A81

Afanasiev, A., Aran, A., Vainio, R., et al. 2018, in Solar Particle Radiation Storms Forecasting and Analysis, eds. O. E. Malandraki, \& N. B. Crosby, Astrophys. Space Sci. Lib., 444, 157

Agostinelli, S., Allison, J., Amako, K., et al. 2003, Nucl. Instrum. Methods, 506, A250

Agueda, N., \& Lario, D. 2016, ApJ, 829, 131

Agueda, N., Vainio, R., Dalla, S., Lario, D., \& Sanahuja, B. 2013, ApJ, 765, 83 Antonucci, E., Romoli, M., Andretta, V., et al. 2020, A\&A, 642, A10 (Solar Orbiter SI)

Aran, A. 2007, PhD Thesis, Universitat de Barcelona, Barcelona, Spain

Aran, A., Sanahuja, B., \& Lario, D. 2005, Ann. Geophys., 23, 3047

Aran, A., Sanahuja, B., \& Lario, D. 2006, AdSpR, 37, 1240

Aran, A., Agueda, N., Afanasiev, A., \& Sanahuja, B. 2018, in Solar Particle Radiation Storms Forecasting and Analysis, eds. O. E. Malandraki, \& N. B. Crosby, Astrophys. Space Sci. Lib., 444, 63

Auchère, F., Andretta, V., Antonucci, E., et al. 2020, A\&A, 642, A6 (Solar Orbiter SI)

Battarbee, M., Vainio, R., Laitinen, T., \& Hietala, H. 2013, A\&A, 558, A110

Battarbee, M., Dalla, S., \& Marsh, M. S. 2018, ApJ, 854, 23

Birks, J., \& Firk, F. 1965, Phys. Today, 18, 60

Bryant, D. A., Cline, T. L., Desai, U. D., \& McDonald, F. B. 1962, J. Geophys. Res., 67, 4983

Bučík, R., Innes, D. E., Mason, G. M., et al. 2018, ApJ, 852, 76

Bučík, R., Innes, D. E., Guo, L., Mason, G. M., \& Wiedenbeck, M. E. 2015, ApJ, 812,53

Bučík, R., Innes, D. E., Mason, G. M., \& Wiedenbeck, M. E. 2016, ApJ, 833, 63 Cane, H. V., McGuire, R. E., \& von Rosenvinge, T. T. 1986, ApJ, 301, 448

Cane, H. V., Reames, D. V., \& von Rosenvinge, T. T. 1988, J. Geophys. Res., 93, 9555

Cane, H. V., Richardson, I. G., \& von Rosenvinge, T. T. 2010, J. Geophys. Res. (Space Phys.), 115, A08101

Chenette, D. L., Chen, J., Clayton, E., et al. 1994, IEEE Trans. Nucl. Sci., 41, 2332

Crosby, N., Heynderickx, D., Jiggens, P., et al. 2015, Space Weather, 13, 406

Dalla, S., Marsh, M. S., Zelina, P., \& Laitinen, T. 2017, A\&A, 598, A73

Desai, M., \& Giacalone, J. 2016, Liv. Rev. Sol. Phys., 13, 3

Dierckxsens, M., Tziotziou, K., Dalla, S., et al. 2015, Sol. Phys., 290, 841

Dresing, N., Gómez-Herrero, R., Klassen, A., et al. 2012, Sol. Phys., 281, 281

Dresing, N., Gómez-Herrero, R., Heber, B., et al. 2014, A\&A, 567, A27

Dröge, W., Kartavykh, Y. Y., Klecker, B., \& Kovaltsov, G. A. 2010, ApJ, 709, 912

Emslie, A. G., Dennis, B. R., Shih, A. Y., et al. 2012, ApJ, 759, 71

Ergun, R. E., Larson, D., Lin, R. P., et al. 1998, ApJ, 503, 435

Fisk, L. A., \& Gloeckler, G. 2008, ApJ, 686, 1466

Fox, N. J., Velli, M. C., Bale, S. D., et al. 2016, Space Sci. Rev., 204, 7

García-Marirrodriga, C., Pacros, A., Strandmoe, S., et al. 2020, A\&A, in press, https://doi.org/10.1051/0004-6361/202038519 (Solar Orbiter SI)

Gevin, O., Delagnes, E., Huynh, D., Limousin, O., \& Lugiez, F. 2015, 2015 IEEE

Nuclear Science Symposium and Medical Imaging Conference (NSS/MIC), 1

Gómez-Herrero, R., Malandraki, O., Dresing, N., et al. 2011, J. Atmos. Sol. Terr. Phys., 73, 551

Gómez-Herrero, R., Dresing, N., Klassen, A., et al. 2015, ApJ, 799, 55

Gómez-Herrero, R., Dresing, N., Klassen, A., et al. 2017, ApJ, 840, 85

Gopalswamy, N., Yashiro, S., Michałek, G., et al. 2002, ApJ, 572, L103

Gopalswamy, N., Xie, H., Yashiro, S., et al. 2012, Space Sci. Rev., 171, 23 
Hasselmann, K., \& Wibberenz, G. 1970, ApJ, 162, 1049

He, H.-Q., Zhou, G., \& Wan, W. 2017, ApJ, 842, 71

Heras, A. M., Sanahuja, B., Lario, D., et al. 1995, ApJ, 445, 497

Horbury, T. S., O’Brien, H., Carrasco Blazquez, I., et al. 2020, A\&A, 642, A9 (Solar Orbiter SI)

Howard, R. A., Vourlidas, A., Colaninno, R. C., et al. 2020, A\&A, 642, A13 (Solar Orbiter SI)

Hu, J., Li, G., Ao, X., Zank, G. P., \& Verkhoglyadova, O. 2017, J. Geophys. Res. (Space Phys.), 122, 10

Jiggens, P., Chavy-Macdonald, M.-A., Santin, G., et al. 2014, J. Space Weather Space Clim., 4, A20

Kahler, S. W. 2001, J. Geophys. Res., 106, 20947

Kahler, S. W., Reames, D. V., \& Sheeley, Jr., N. R. 2001, ApJ, 562, 558

Kallenrode, M.-B. 1993a, J. Geophys. Res., 98, 5573

Kallenrode, M.-B. 1993b, J. Geophys. Res., 98, 19

Kallenrode, M.-B., Cliver, E. W., \& Wibberenz, G. 1992, ApJ, 391, 370

Kartavykh, Y. Y., Dröge, W., \& Gedalin, M. 2016, ApJ, 820, 24

Khabarova, O. V., Malandraki, O. E., Zank, G. P., et al. 2018, in Space Weathe of the Heliosphere: Processes and Forecasts, eds. C. Foullon, \& O. E. Malandraki, IAU Symp., 335, 75

Klecker, B., Hovestadt, D., Scholer, M., et al. 1984, ApJ, 281, 458

Klecker, B., Möbius, E., \& Popecki, M. A. 2007, Space Sci. Rev., 130, 273

Klein, K.-L., \& Dalla, S. 2017, Space Sci. Rev., 212, 1107

Kocharov, L., Vainio, R., Kovaltsov, G. A., \& Torsti, J. 1998, Sol. Phys., 182, 195

Kocharov, L., Pohjolainen, S., Mishev, A., et al. 2017, ApJ, 839, 79

Kontar, E. P. 2001, A\&A, 375, 629

Kontar, E. P., \& Reid, H. A. S. 2009, ApJ, 695, L140

Kontar, E. P., Yu, S., Kuznetsov, A. A., et al. 2017, Nat. Commun., 8, 1515

Kozarev, K., Schwadron, N. A., Dayeh, M. A., et al. 2010, Space Weather, 8 , S00E08

Kozarev, K. A., Evans, R. M., Schwadron, N. A., et al. 2013, ApJ, 778, 43

Krucker, S., Kontar, E. P., Christe, S., \& Lin, R. P. 2007, ApJ, 663, L109

Krucker, S., Hurford, G. J., Grimm, O., et al. 2020, A\&A, 642, A15 (Solar Orbiter SI)

Kucharek, H., MöBius, E., Li, W., et al. 2003, J. Geophys. Res. (Space Phys.), 108,8040

Laitinen, T., Kopp, A., Effenberger, F., Dalla, S., \& Marsh, M. S. 2016, A\&A, 591, A 18

Lario, D., Sanahuja, B., \& Heras, A. M. 1998, ApJ, 509, 415

Lario, D., Kallenrode, M.-B., Decker, R. B., et al. 2006, ApJ, 653, 1531

Lario, D., Aran, A., Agueda, N., \& Sanahuja, B. 2007, AdSpR, 40, 289

Lario, D., Aran, A., Gómez-Herrero, R., et al. 2013, ApJ, 767, 41

Lario, D., Raouafi, N. E., Kwon, R.-Y., et al. 2014, ApJ, 797, 8

Lario, D., Kwon, R.-Y., Vourlidas, A., et al. 2016, ApJ, 819, 72

le Roux, J. A., Zank, G. P., Webb, G. M., \& Khabarova, O. 2015, ApJ, 801, 112

le Roux, J. A., Zank, G. P., Webb, G. M., \& Khabarova, O. V. 2016, ApJ, 827 47

Lee, M. A., Mewaldt, R. A., \& Giacalone, J. 2012, Space Sci. Rev., 173, 247

Lin, R. P., Curtis, D. W., Larson, D. E., et al. 2008, Space Sci. Rev., 136, 241

Luhmann, J. G., Mays, M. L., Odstrcil, D., et al. 2017, Space Weather, 15, 934

Maksimovic, M., Bale, S. D., Chust, T., et al. 2020, A\&A, 642, A12 (Sola Orbiter SI)

Malandraki, O. E., Sarris, E. T., Lanzerotti, L. J., et al. 2002, J. Atmos. Sol. Terr. Phys., 64, 517

Malandraki, O. E., Sarris, E. T., \& Tsiropoula, G. 2003, Ann. Geophys., 21, 1249

Malandraki, O. E., Lario, D., Lanzerotti, L. J., et al. 2005, J. Geophys. Res. (Space Phys.), 110, A09S06

Malandraki, O. E., \& Crosby, N. B. 2018, in Solar Particle Radiation Storms Forecasting and Analysis, Astrophys. Space Sci. Lib., 444

Marsh, M. S., Dalla, S., Dierckxsens, M., Laitinen, T., \& Crosby, N. B. 2015 Space Weather, 13, 386

Mason, G. M. 2007, Space Sci. Rev., 130, 231

Mason, G. M., Gold, R. E., Krimigis, S. M., et al. 1998, Space Sci. Rev., 86, 409

Mason, G. M., Korth, A., Walpole, P. H., et al. 2008, Space Sci. Rev., 136, 257

Mason, G. M., Li, G., Cohen, C. M. S., et al. 2012, ApJ, 761, 104

Masson, S., Klein, K.-L., Bütikofer, R., et al. 2009, Sol. Phys., 257, 305

McKenna-Lawlor, S., Bhardwaj, A., Ferrari, F., et al. 2015, Acta Astron., 109, 182

Melcher, C., Schweitzer, J., Liberman, A., \& Simonetti, J. 1985, IEEE Trans Nucl. Sci., 32, 529

Mewaldt, R. A. 2006, Space Sci. Rev., 124, 303

Müller-Mellin, R., Kunow, H., Fleißner, V., et al. 1995, Sol. Phys., 162, 483

Müller-Mellin, R., Böttcher, S., Falenski, J., et al. 2008, Space Sci. Rev., 136, 363

Müller, D., Marsden, R. G., St. Cyr, O. C., Gilbert, H. R., \& The Solar Orbiter Team 2013, Sol. Phys., 285, 25
Müller, D., St. Cyr, O. C., Zouganelis, I., et al. 2020, A\&A, 642, A1 (Solar Orbiter SI)

Neergaard Parker, L., \& Zank, G. P. 2012, ApJ, 757, 97

Nitta, N. V., Reames, D. V., De Rosa, M. L., et al. 2006, ApJ, 650, 438

NuDat, N. 2011, Brookhaven National Laboratory

Owen, C. J., Bruno, R., Livi, S., et al. 2020, A\&A, 642, A16 (Solar Orbiter SI)

Pacheco, D., Agueda, N., Gómez-Herrero, R., \& Aran, A. 2017, J. Space Weather Space Clim., 7, A30

Pacheco, D., Agueda, N., Aran, A., Heber, B., \& Lario, D. 2019, A\&A, 624, A3

Pick, M., \& Vilmer, N. 2008, A\&ARv, 16, 1

Pomoell, J., Aran, A., Jacobs, C., et al. 2015, J. Space Weather Space Clim., 5, A12

Porsche, H. 1977, J. Geophys, Z. Geophys., 42, 551

Posner, A. 2007, Nucl. Instrum. Methods, 5

Reames, D. V. 1999, Space Sci. Rev., 90, 413

Reames, D. V. 2013, Space Sci. Rev., 175, 53

Reames, D. V. 2017, Solar Energetic Particles (Berlin: Springer)

Reames, D. V. 2018, Space Sci. Rev., 214, 61

Reames, D. V., Meyer, J. P., \& von Rosenvinge, T. T. 1994, ApJS, 90, 649

Reid, H. A. S., \& Kontar, E. P. 2013, Sol. Phys., 285, 217

Richardson, I. G. 2004, Space Sci. Rev., 111, 267

Richardson, I. G. 2018, Liv. Rev. Sol. Phys., 15, 1

Richardson, I. G., von Rosenvinge, T. T., Cane, H. V., et al. 2014, Sol. Phys. 289, 3059

Rochus, P., Auchère, F., Berghmans, D., et al. 2020, A\&A, 642, A8 (Solar Orbiter SI)

Rodríguez-Gasén, R., Aran, A., Sanahuja, B., Jacobs, C., \& Poedts, S. 2014, Sol. Phys., 289, 1745

Rodríguez-Pacheco, J., Sequeiros, J., del Peral, L., Bronchalo, E., \& Cid, C. 1998, Sol. Phys., 181, 185

Rouillard, A. P., Pinto, R. F., Vourlidas, A., et al. 2020, A\&A, 642, A2 (Solar Orbiter SI)

Ruffolo, D. 1995, ApJ, 442, 861

Schlickeiser, R. 1989, ApJ, 336, 243

Schwadron, N. A., Gorby, M., Török, T., et al. 2014, Space Weather, 12, 323

Schwadron, N. A., Townsend, L., Kozarev, K., et al. 2010, Space Weather, 8, SO0E02

Solanki, S. K., del Toro Iniesta, J. C., Woch, J., et al. 2020, A\&A, 642, A11 (Solar Orbiter SI)

SPICE Consortium (Anderson, M., et al.) 2020, A\&A, 642, A14 (Solar Orbiter $\mathrm{SI})$

Strauss, R. D. T., Dresing, N., \& Engelbrecht, N. E. 2017, ApJ, 837, 43

Sullivan, J. 1971, Nucl. Instrum. Methods, 95, 5

Tammen, J., Elftmann, R., Kulkarni, S. R., Böttcher, S. I., \& WimmerSchweingruber, R. F. 2015, Nucl. Instrum. Methods Phys. Res. B, 360, 129

Tan, L. C., Malandraki, O. E., Reames, D. V., et al. 2012, ApJ, 750, 146

Taut, A., Berger, L., Drews, C., \& Wimmer-Schweingruber, R. F. 2015, A\&A, 576, A55

Tindall, C. S., Palaio, N. P., Ludewigt, B. A., et al. 2008, IEEE Trans. Nucl. Sci., 55,797

Trottet, G., Samwel, S., Klein, K.-L., Dudok de Wit, T., \& Miteva, R. 2015, Sol. Phys., 290, 819

Tylka, A. J., \& Lee, M. A. 2006, ApJ, 646, 1319

Tylka, A. J., Cohen, C. M. S., Dietrich, W. F., et al. 2005, ApJ, 625, 474

Tylka, A. J., Malandraki, O. E., Dorrian, G., et al. 2013, Sol. Phys., 285, 251

Vainio, R., \& Afanasiev, A. 2018, in Solar Particle Radiation Storms Forecasting and Analysis, eds. O. E. Malandraki, \& N. B. Crosby, Astrophys. Space Sci. Lib., 444, 45

Vainio, R., \& Laitinen, T. 2007, ApJ, 658, 622

Vainio, R., Agueda, N., Aran, A., \& Lario, D. 2007, in Space Weather: Research Towards Applications in Europe 2nd European Space Weather Week (ESWW2), ed. J. Lilensten, Astrophys. Space Sci. Lib., 344, 27

Vainio, R., Desorgher, L., Heynderickx, D., et al. 2009, Space Sci. Res., 147, 187

Vainio, R., Pönni, A., Battarbee, M., et al. 2014, J. Space Weather Space Clim., 4, A08

Velli, M., Harra, L. K., Vourlidas, A., et al. 2020, A\&A, 642, A4 (Solar Orbiter SI)

Voshchepynets, A., Krasnoselskikh, V., Artemyev, A., \& Volokitin, A. 2015, ApJ, 807, 38

Walsh, A. P., Horbury, T. S., Maksimovic, M., et al. 2020, A\&A, 642, A5 (Solar Orbiter SI)

Weber, S., Christ, D., Kurzeja, M., et al. 2003, IEEE Trans. Nucl. Sci., 50, 1370

Wibberenz, G., \& Cane, H. V. 2006, ApJ, 650, 1199

Wiedenbeck, M. E., Mason, G. M., Cohen, C. M. S., et al. 2013, ApJ, 762, 54

Wijsen, N., Aran, A., Pomoell, J., \& Poedts, S. 2019, A\&A, 622, A28

Zank, G. P., le Roux, J. A., Webb, G. M., Dosch, A., \& Khabarova, O. 2014, ApJ, 797,28 
Zank, G. P., Hunana, P., Mostafavi, P., et al. 2015, ApJ, 814, 137

Zhang, M., Qin, G., \& Rassoul, H. 2009, ApJ, 692, 109

Zouganelis, I., De Groof, A., Walsh, A. P., et al. 2020, A\&A, 642, A3 (Solar Orbiter SI)

${ }^{1}$ Universidad de Alcalá, Space Research Group, 28805 Alcalá de Henares, Spain

e-mail: javier.pacheco@uah.es

2 Institut für Experimentelle und Angewandte Physik, ChristianAlbrechts-Universität zu Kiel, Kiel, Germany

3 Johns Hopkins University Applied Physics Laboratory, Laurel, MD USA

${ }^{4}$ University of Colorado/LASP, Boulder, CO, USA

5 Departament de Física Quàntica i Astrofísica, Institut de Ciències del Cosmos (ICCUB), Universitat de Barcelona, Barcelona, Spain

${ }^{6}$ Imperial College London, UK

7 ESA-ESAC, Madrid, Spain

8 Observatoire de Paris-Meudon, France

9 Karl-Franzens-Universität Graz, Austria

${ }^{10}$ Institute of Atmospheric Physics, Prague, Czech Republic
11 University of New Hampshire, Durham, USA

12 NASA HQ, Washington DC, USA

13 University of Peking, PR China

14 ESA-ESTEC, Noordwijk, The Netherlands

15 Jet Propulsion Laboratory, Pasadena, CA, USA

16 Max-Planck-Institut für extraterrestrische Physik, Garching, Germany

17 School of Physics \& Astronomy, University of Glasgow, UK

18 Windisch, Switzerland \& SSL, UC, Berkeley, USA

19 National Observatory of Athens/IAASARS, Greece

${ }^{20}$ University College, London, UK

${ }^{21}$ University of Turku, Turku, Finland

22 University of Central Lancashire, UK

23 University of Würzburg, Germany

${ }^{24}$ Commissariat à l'énergie atomique, CEA Saclay, France

25 Institute of Experimental Physics, Slovak Academy of Sciences, Slovakia

26 Goddard Space Flight Center,Greenbelt, MD, USA

27 Leibniz-Institut für Astrophysik Potsdam (AIP), Potsdam, Germany

${ }^{28}$ Ioffe Physical-Technical Institute, St-Petersburg, Russia 